Supporting Information

for

\title{
Heavy-Atom Tunneling Processes During Denitrogenation of a Diazabicycloheptene and Ring Closure of Cyclopentane-1,3-diyl Diradical. Stereoselectivity in Tunneling and Matrix Effect
}

\author{
Sujan K. Sarkar,*,+, ${ }^{*}$ Ephrath Solel, ${ }^{\#, \ddagger}$ Sebastian Kozuch,,*, and Manabu Abe*,,,\$,§ \\ ${ }^{\dagger}$ Department of Chemistry, Graduate School of Science, Hiroshima University, Hiroshima 739-8526, Japan \\ "Department of Chemistry, Ben-Gurion University of the Negev, Beer-Sheva 841051, Israel \\ ${ }^{\text {s} H i r o s h i m a ~ U n i v e r s i t y ~ R e s e a r c h ~ C e n t e r ~ f o r ~ P h o t o-D r u g-D e l i v e r y ~ S y s t e m s ~(H i U-P-D D S), ~ H i r o s h i m a ~ U n i v e r s i t y, ~}$ \\ Hiroshima 739-8526, Japan \\ §JST-CREST, K's Gobancho 6F, 7, Gobancho, Chiyoda-ku, Tokyo 102-0075, Japan \\ *Sujan K. Sarkar: sujankumar123@gmail.com \\ *Sebastian Kozuch: kozuch@bgu.ac.il \\ *Manabu Abe: mabe@hiroshima-u.ac.jp \\ ${ }^{*}$ S.K.S. and E.S. contributed equally.
}

\section{Table of Contents}

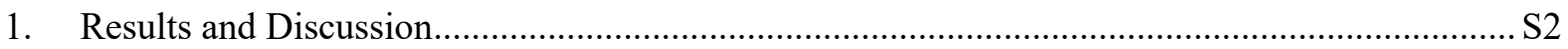

1.1. Low-Temperature EPR Spectroscopy in Glassy Organic Matrices........................................ S2

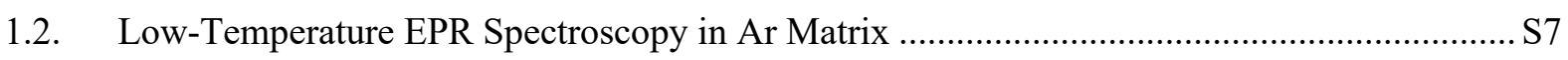

1.3. Wavelength Dependent Product Distribution ............................................................................ S9

1.4. Photoproducts' Stereoselectivity at Room Temperature in Solution (Characterization of CP) S10

1.5. Photoproducts' Stereoselectivity in Low-Temperature Photolysis in Ar Matrix ................... S13

1.6. Spin State and Temperature Effects on Stereoselectivity in Solution .................................... S13

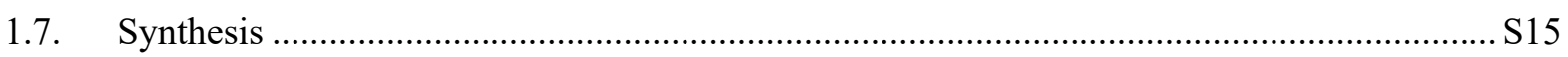

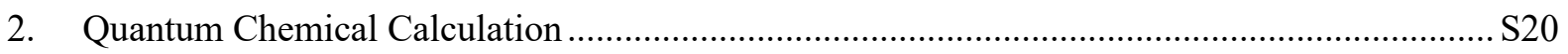

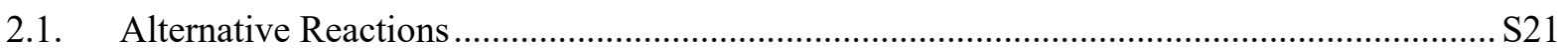

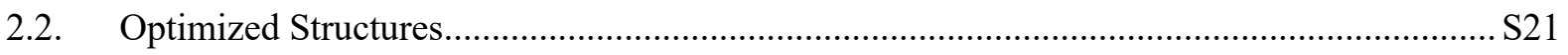

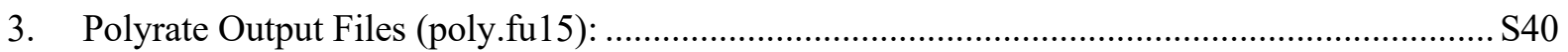

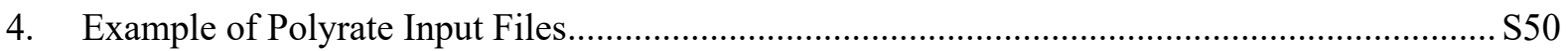

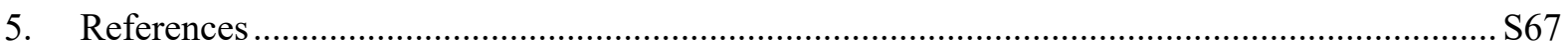




\section{Results and Discussion}

Further references are given on the photolysis of $\mathbf{A Z} .^{1-6}$

\subsection{Low-Temperature EPR Spectroscopy in Glassy Organic Matrices}

\section{Stretch Exponential Equation}

The decay curve showing a decrease in the intensity of the signal of T-DR could not be fitted using a simple single exponential equation to obtain a decay rate constant. When a product is formed via out-of-plane motion of T-DR, different host sites in the solid matrix rearrange in different ways. ${ }^{7}$ Therefore, the decay of T-DR follows dispersive kinetics due to the arbitrary distribution of these sites. Weak interactions between the hosts and the substrates also exist in the matrix, which result in further distributions of rate constants. Due to this, the decrease in intensity of the $2840 \mathrm{G}$ signal was fitted using the stretched exponential approach introduced by Siebrand and Wildman. ${ }^{8}$ In Eq. (1), $\beta$ is the dispersion coefficient, $k$ is the rate constant, $\mathrm{C}$ is a constant, and $I_{\mathrm{t}}$ and $I_{0}$ are the intensities at times $t$ and 0 , respectively. The dispersive coefficients obtained after fitting the decay at 5, 9, 13, 17, 25 , and $30 \mathrm{~K}$ are $0.67511,0.68476,0.60779,0.65907,0.63449$, and 0.66202 respectively.

$$
I_{\mathrm{t}}=I_{0} \mathrm{e}^{\left(-(k \mathrm{t})^{\beta}\right)}+\mathrm{C} \quad(0<\beta<1)
$$

Figure S1. Decay of EPR signal of T-DR at $2840 \mathrm{G}$ in glassy MTHF matrix at $13 \mathrm{~K}$ under dark conditions. The decay was fitted using simple single exponential equation.

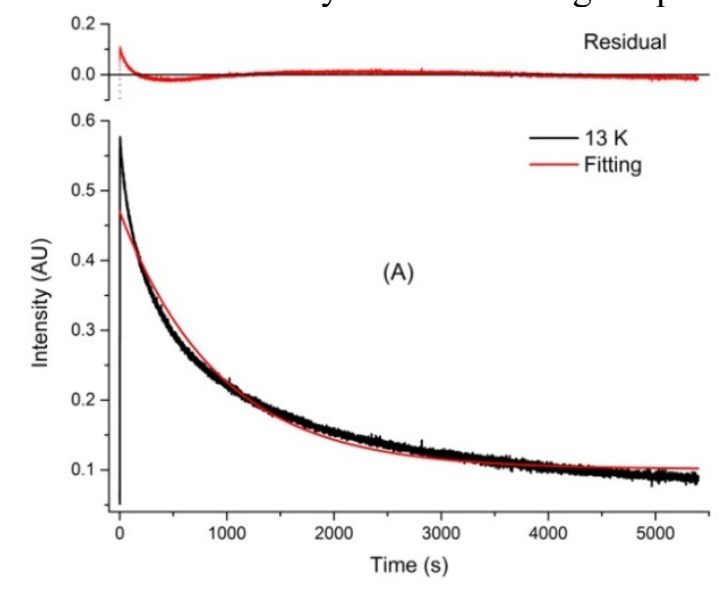

Figure S2. Decay of EPR signal of T-DR at $2840 \mathrm{G}$ in glassy MTHF matrix at $5 \mathrm{~K}$ under dark conditions. The decay was fitted using stretched exponential (left) and simple single exponential (right) equations. 

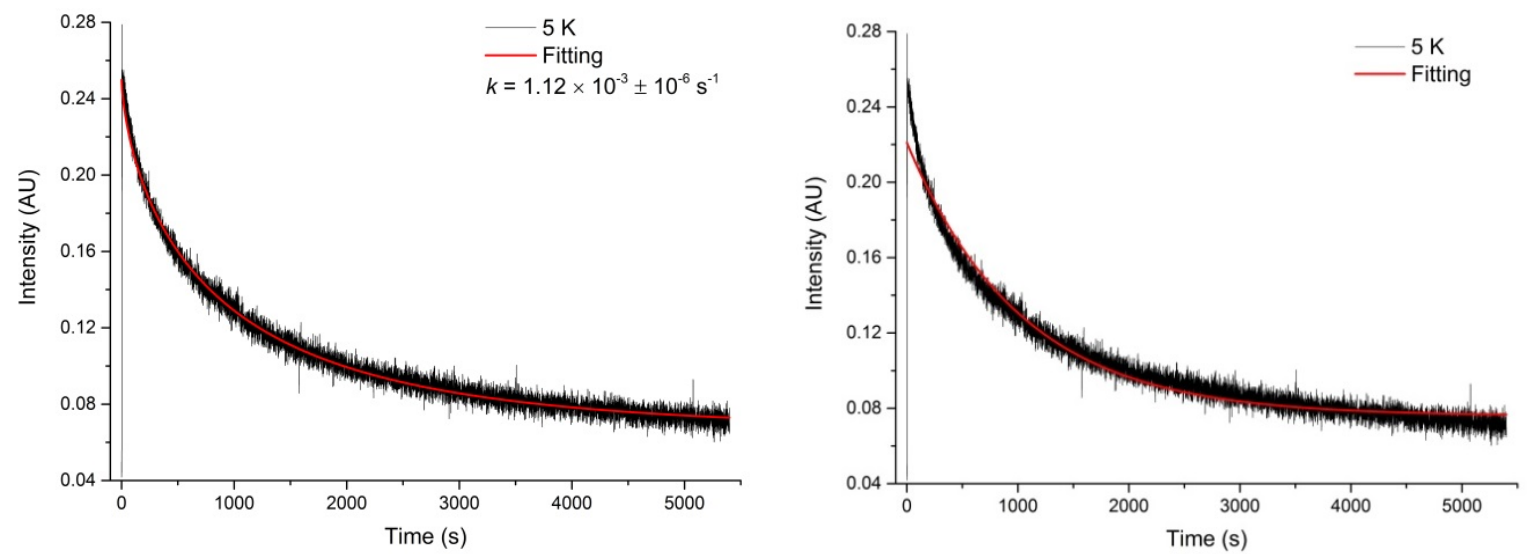

Figure S3. Decay of EPR signal of T-DR at $2840 \mathrm{G}$ in glassy MTHF matrix at $9 \mathrm{~K}$ under dark conditions. The decay was fitted using stretched exponential (left) and simple single exponential (right) equations.
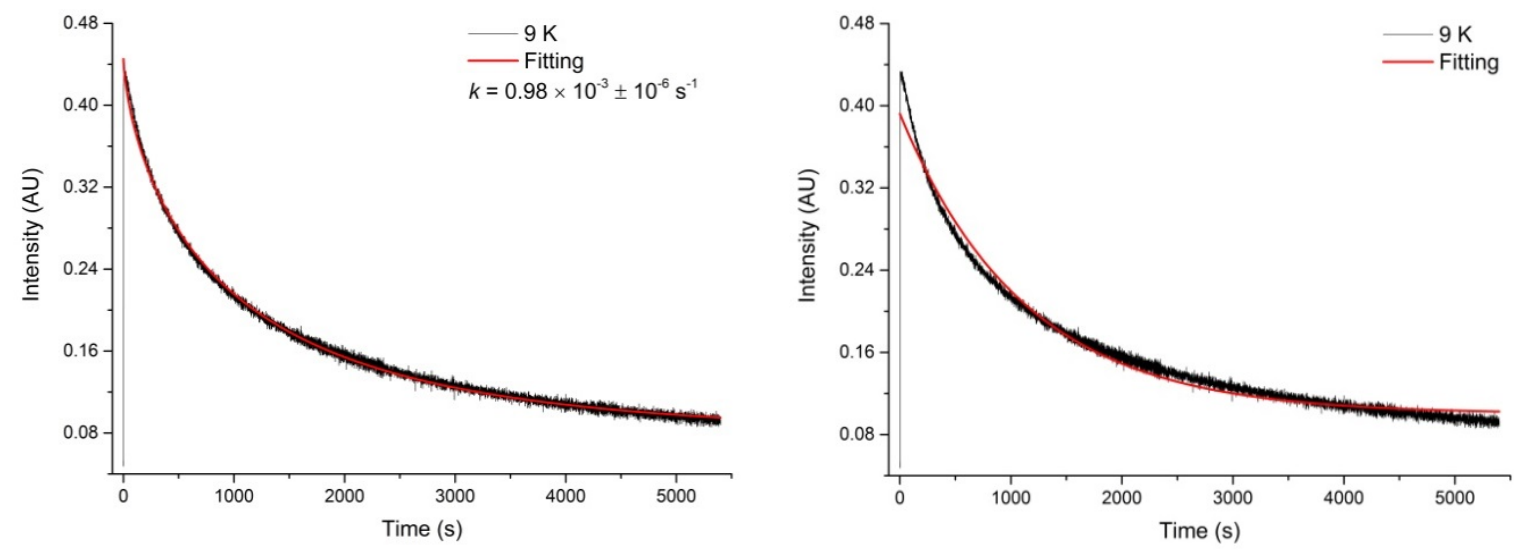

Figure S4. Decay of EPR signal of T-DR at $2840 \mathrm{G}$ in glassy MTHF matrix at $17 \mathrm{~K}$ under dark conditions. The decay was fitted using stretched exponential (left) and simple single exponential (right) equations.
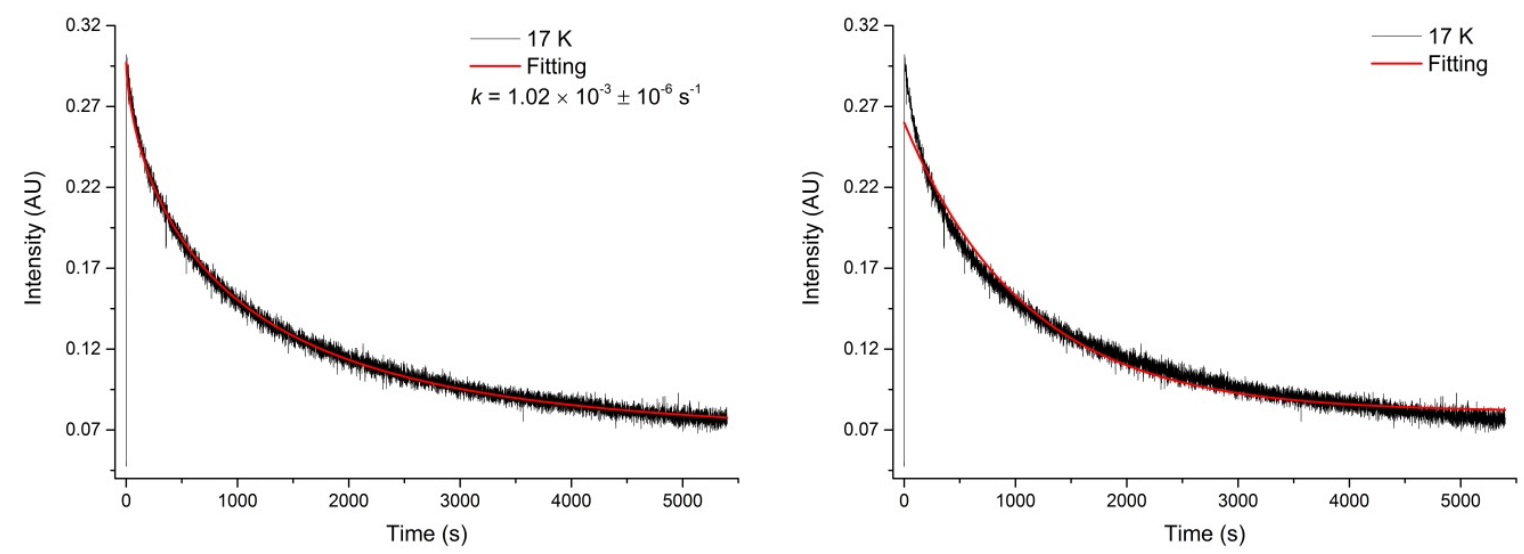

Figure S5. Decay of EPR signal of T-DR at $2840 \mathrm{G}$ in glassy MTHF matrix at $25 \mathrm{~K}$ under dark conditions. The decay was fitted using stretched exponential (left) and simple single exponential (right) equations. 

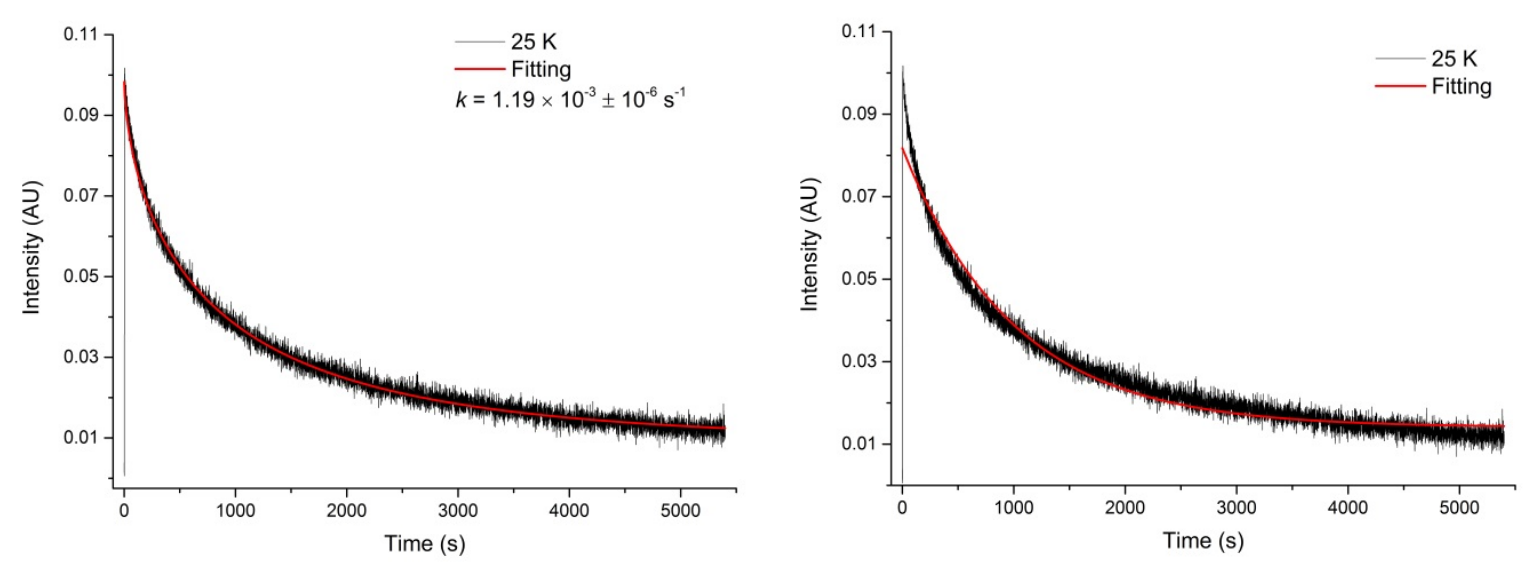

Figure S6. Decay of EPR signal of T-DR at $2840 \mathrm{G}$ in glassy MTHF matrix at $30 \mathrm{~K}$ under dark conditions. The decay was fitted using stretched exponential (left) and simple single exponential (right) equations.
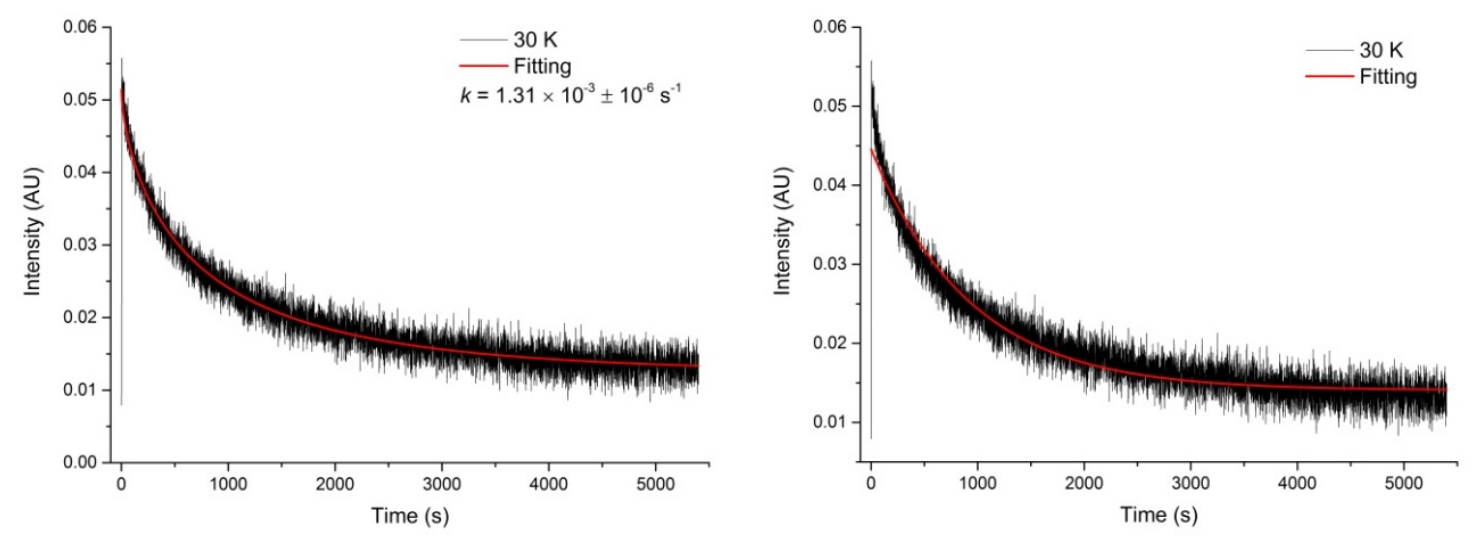

Figure S7. Decay rates of EPR signal of T-DR at $2840 \mathrm{G}$ in glassy MTHF matrix at different temperatures were used to obtain an Arrhenius plot of $\ln (k)$ vs. 1/T.

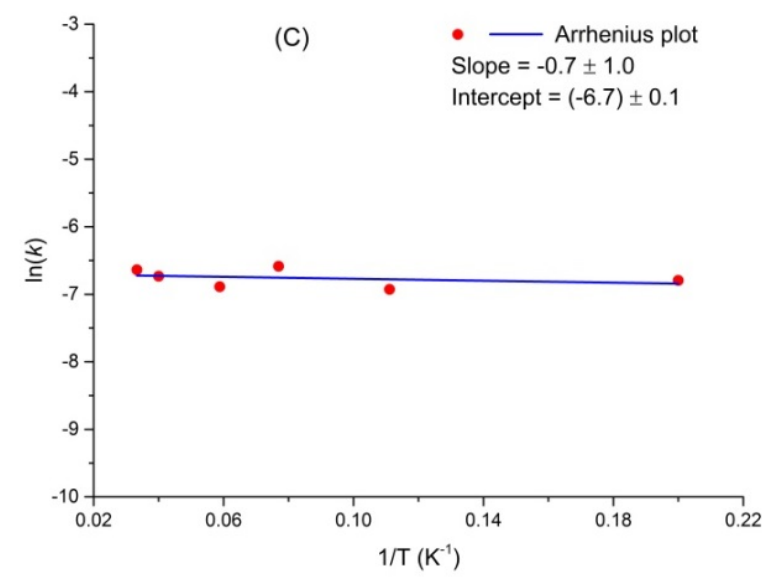

We obtained the thermodynamic parameters of T-DR decay in glassy MTHF using the Eyring equation. Assuming that the reaction goes through a transition state $(\kappa=1)$, the slope and intercept of an Eyring plot (Figure S8) yielded the standard enthalpy $\left(\Delta^{*} \mathrm{H}^{\circ}=-79.8 \mathrm{~J} \mathrm{~mol}^{-1}\right)$ and standard entropy $\left(\Delta^{\ddagger} \mathrm{S}^{\circ}=-282.3 \mathrm{~J} \mathrm{~mol}^{-1} \mathrm{~K}^{-1}\right)$ of activation, respectively. 
Figure S8. Decay rates of EPR signal of T-DR at $2840 \mathrm{G}$ in glassy MTHF matrix at different temperatures were used to obtain an Eyring plot of $\ln (k / \mathrm{T})$ vs. $1 / \mathrm{T}$.

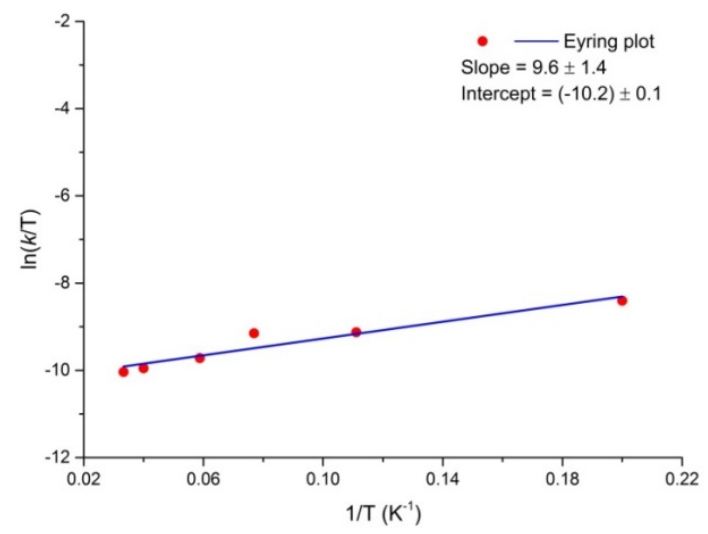

Figure S9. EPR spectrum $(9.40 \mathrm{GHz})$ of T-DR in glassy 3MP at $5 \mathrm{~K}$ during $7 \mathrm{~m}$ photolysis of $0.1 \mathrm{M}$ solution of $\mathbf{A Z}$ using a high-pressure $\mathrm{Hg}$ lamp.

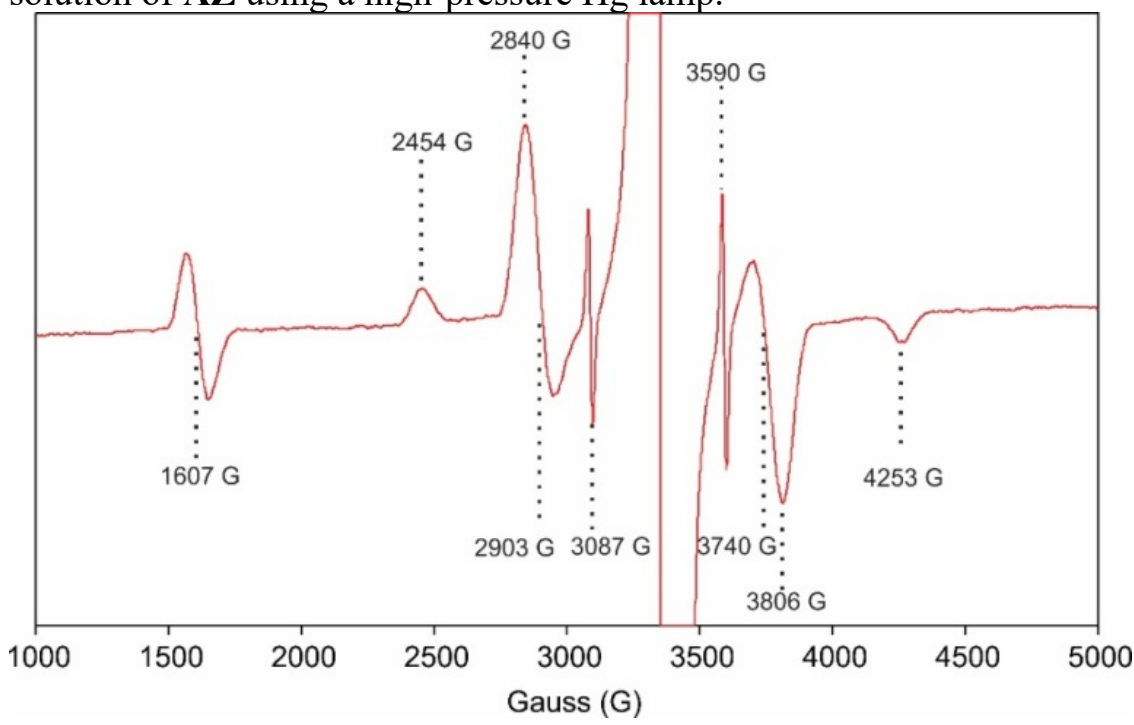

Figure S10. Decay of EPR signal of T-DR at $2840 \mathrm{G}$ in glassy 3MP matrix at 5, 9, 13, 17, 25, and 30 $\mathrm{K}$ under dark conditions. The decay was fitted using simple single exponential equation.
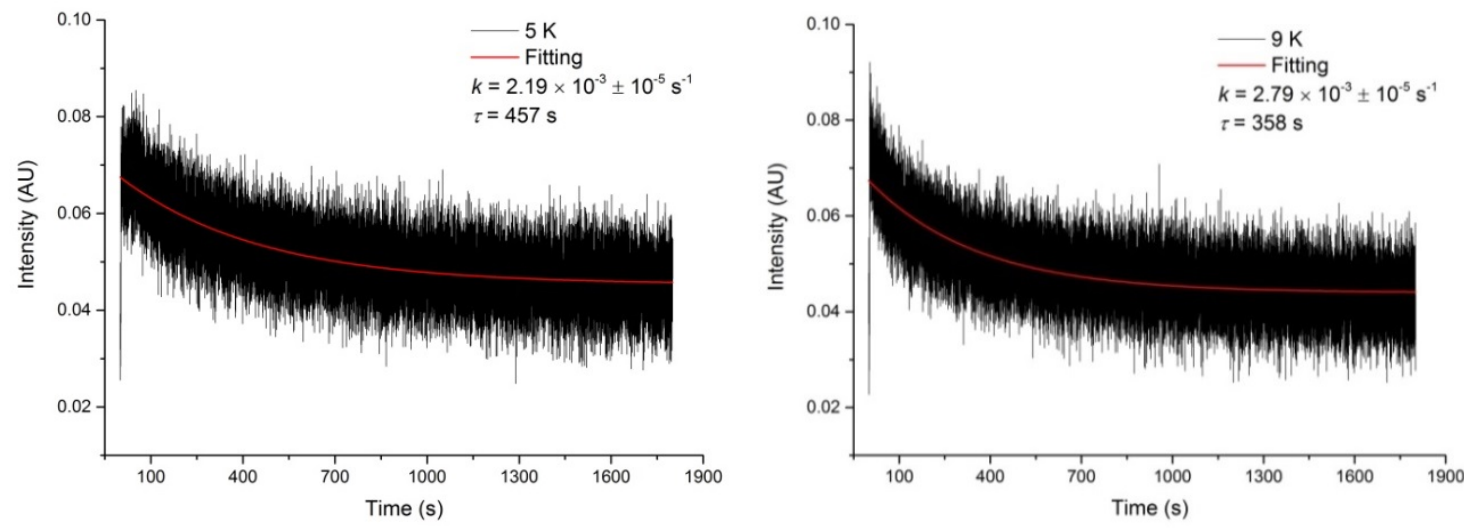

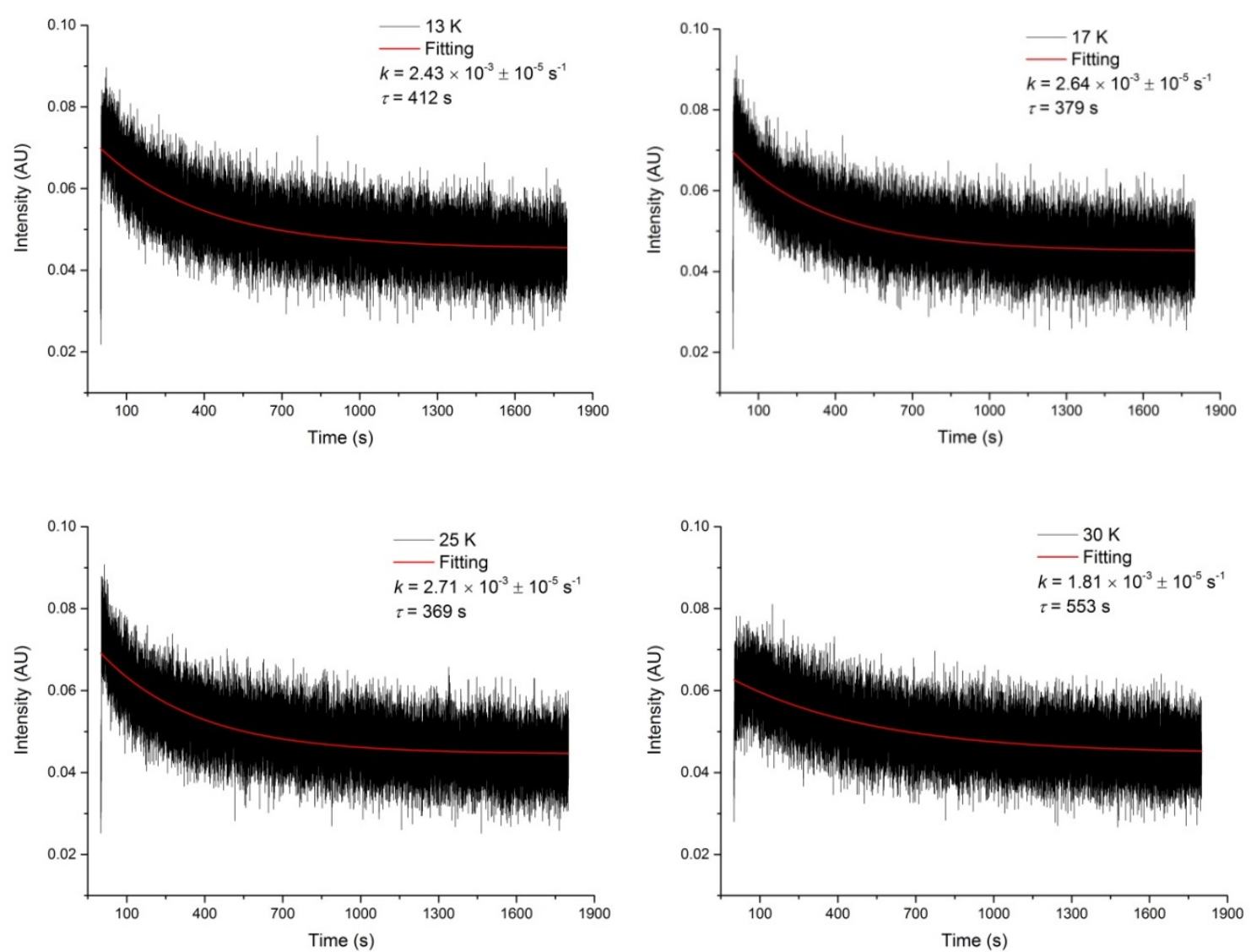

Figure S11. Decay rates of EPR signal of T-DR at $2840 \mathrm{G}$ in glassy $3 \mathrm{MP}$ matrix at different temperatures were used to obtain an Arrhenius plot of $\ln (k)$ vs. 1/T.

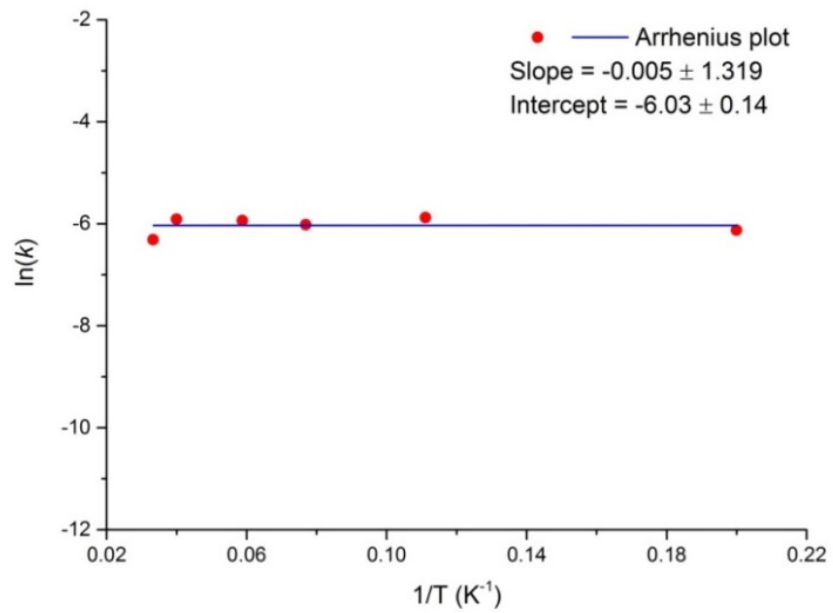

From Eyring plot in 3MP (Figure S12), the slope and intercept yielded the standard enthalpy $\left(\Delta^{\dagger} \mathrm{H}^{\circ}=-85.88 \mathrm{~J} \mathrm{~mol}^{-1}\right)$ and standard entropy $\left(\Delta^{\star} \mathrm{S}^{\circ}=-277.02 \mathrm{~J} \mathrm{~mol}^{-1} \mathrm{~K}^{-1}\right)$ of activation, respectively. The enthalpy of activation of T-DR decay was found to be almost zero in 3MP matrix.

Figure S12. Decay rates of EPR signal of T-DR at $2840 \mathrm{G}$ in glassy $3 \mathrm{MP}$ matrix at different temperatures were used to obtain an Eyring plot of $\ln (k / \mathrm{T})$ vs. $1 / \mathrm{T}$. 


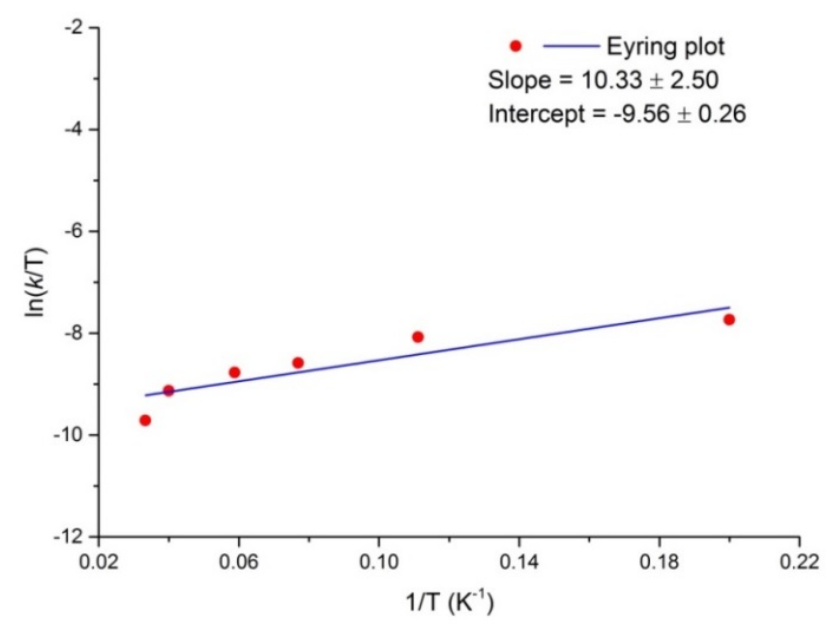

Figure S13. Decay of T-DR- $\mathrm{d}_{6}$ at $2831 \mathrm{G}$ in glassy MTHF matrix at 5 (left) and 9 (right) K under dark conditions. Decay time profiles were fitted using stretched exponential equation [Eq. (1)]. The dispersive coefficients obtained after fitting the decay at 5 and $9 \mathrm{~K}$ are 0.37323 and 0.40864 , respectively
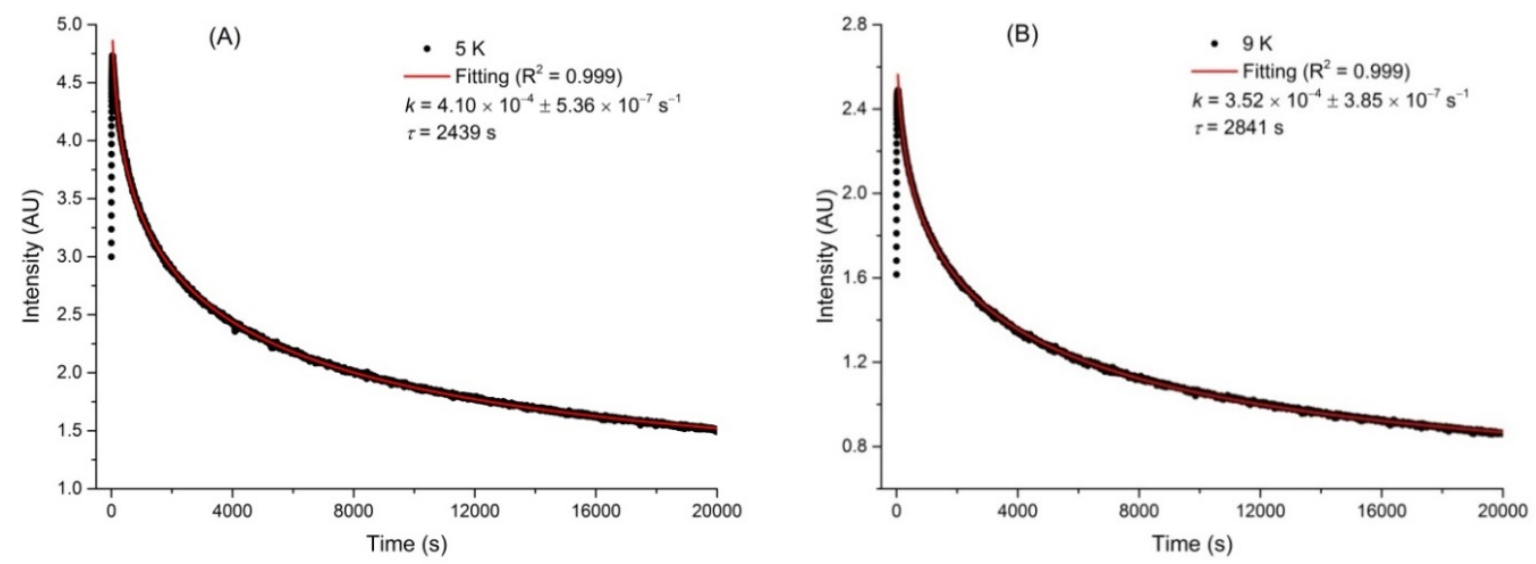

\subsection{Low-Temperature EPR Spectroscopy in Ar Matrix}

Figure S14. Decay of EPR signal of T-DR at $2918 \mathrm{G}$ in Ar matrix at $5 \mathrm{~K}$ (left) and $20 \mathrm{~K}$ (right) under dark conditions. The decay was fitted using simple single exponential equation.
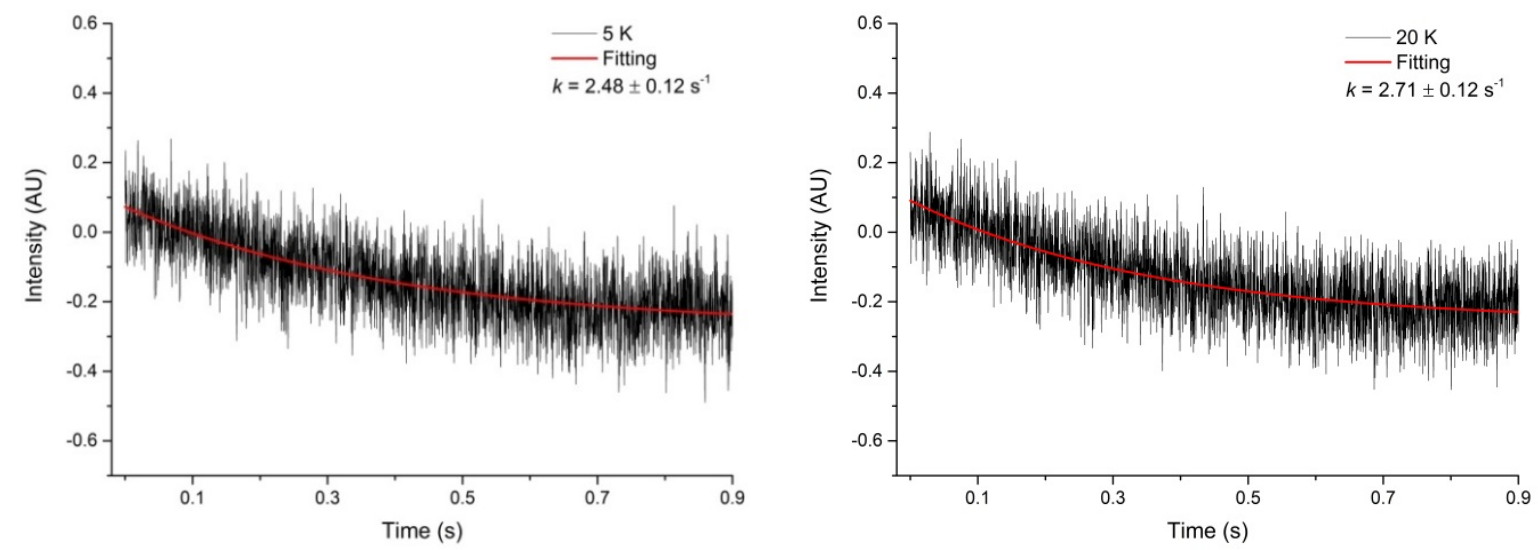
Table S1. Summarized zfs parameters of T-DR and T-DR- $\mathrm{d}_{6}$ in different matrices, and literature value of T-DR in cyclohexane.

\begin{tabular}{|c|c|c|c|}
\hline Species & Matrix & $\begin{array}{c}|D / h \mathbf{c}| \\
\text { in } \mathbf{c m}^{-} \\
1\end{array}$ & $\begin{array}{c}|E / h \mathrm{c}| \\
\text { in } \mathrm{cm}^{-} \\
1\end{array}$ \\
\hline $\begin{array}{c}\text { T-DR } \\
\left(\mathrm{x}_{2}, \mathrm{y}_{2}\right)\end{array}$ & MTHF & 0.0830 & 0.0020 \\
\hline $\begin{array}{c}\text { T-DR } \\
\left(\mathrm{z}_{1}, \mathrm{z}_{2}\right)\end{array}$ & MTHF & 0.0841 & - \\
\hline $\begin{array}{c}\text { T-DR } \\
\left(\mathrm{x}_{2}, \mathrm{y}_{2}\right)\end{array}$ & $3 \mathrm{MP}$ & 0.0830 & 0.0020 \\
\hline $\begin{array}{c}\text { T-DR } \\
\left(\mathrm{x}_{2}, \mathrm{y}_{2}\right)\end{array}$ & $\mathrm{Ar}$ & 0.0835 & 0.0019 \\
\hline $\begin{array}{c}\text { T-DR- } \\
\mathrm{d}_{6}\left(\mathrm{x}_{2},\right. \\
\left.\mathrm{y}_{2}\right)\end{array}$ & MTHF & 0.0855 & 0.0023 \\
\hline $\begin{array}{c}\text { T-DR } \\
(\text { main } \\
\text { text }{ }^{41} \text {, } \\
42 \text { ) }\end{array}$ & Cyclohexane & 0.084 & 0.0020 \\
\hline
\end{tabular}

Table S2. Experimental and calcualted (after scaling factor $=0.9613$ ) IR peaks, and vibrational modes of $\mathbf{A Z}, \mathbf{C P}, \mathbf{A Z}-\mathrm{d}_{6}$, ret-CP-d 6 , and inv-CP-d 6 .

\begin{tabular}{|c|c|c|c|}
\hline Species & $\begin{array}{l}\text { Experimental Peaks } \\
\qquad\left(\mathrm{cm}^{-1}\right)\end{array}$ & $\begin{array}{c}\text { Calculated Peaks } \\
\left(\mathrm{cm}^{-1}\right)\end{array}$ & Modes \\
\hline \multirow[t]{8}{*}{$A Z$} & 1494 & 1540 & $\mathrm{~N}=\mathrm{N}$ stretching \\
\hline & 1446 & 1456 & $\mathrm{H}-\mathrm{C}-\mathrm{H}$ scissoring \\
\hline & 1282 & 1275 & $\mathrm{H}-\mathrm{C}-\mathrm{H}$ wagging \\
\hline & 1260 & 1252 & $\mathrm{~N}-\mathrm{C}-\mathrm{H}$ bending \\
\hline & 1198 & 1184 & $\mathrm{H}-\mathrm{C}-\mathrm{H}$ twisting \\
\hline & 1122 & 1106 & $\mathrm{H}-\mathrm{C}-\mathrm{H}$ twisting \\
\hline & 950 & 924 & C-C stretching \\
\hline & 884 & 859 & C-C stretching \\
\hline \multirow[t]{6}{*}{ CP } & 1275 & 1272 & $\mathrm{H}-\mathrm{C}-\mathrm{H}$ wagging \\
\hline & 1049 & 1049 & $\mathrm{H}-\mathrm{C}-\mathrm{H}$ wagging \\
\hline & 968 & 960 & $\mathrm{H}-\mathrm{C}-\mathrm{C}-\mathrm{H}$ twisting \\
\hline & 916 & 911 & $\mathrm{H}-\mathrm{C}-\mathrm{C}-\mathrm{H}$ scissoring \\
\hline & 786 & 769 & C-C stretching \\
\hline & 761 & 748 & $\mathrm{H}-\mathrm{C}-\mathrm{C}-\mathrm{H}$ scissoring \\
\hline \multirow[t]{7}{*}{ AZ- $\mathrm{d}_{6}$} & 1482 & 1531 & $\mathrm{~N}=\mathrm{N}$ stretching \\
\hline & 1306 & 1313 & H-C-D scissoring \\
\hline & 1300 & 1303 & $\mathrm{H}-\mathrm{C}-\mathrm{C}-\mathrm{H}$ rocking \\
\hline & 1244 & 1235 & $\mathrm{H}-\mathrm{C}-\mathrm{C}-\mathrm{H}$ rocking \\
\hline & 1161 & 1148 & D-C-D scissoring \\
\hline & 1144 & 1121 & C-C stretching \\
\hline & 961 & 944 & D-C-C-D twisting \\
\hline
\end{tabular}




\begin{tabular}{cccc}
\hline & 821 & 802 & D-C--C-D wagging \\
& 812 & 795 & D-C-D rocking \\
\hline ret-CP-d $_{6}$ & 820 & 839 & H-C-C-H coupled with D-C-C-D scissoring \\
& & & D-C-C-D scissoring \\
& 788 & 771 & D-C-C-D twisting \\
& 656 & 636 & D-C-C-D scissoring \\
& 583 & 570 & \\
\hline inv-CP-d $_{6}$ & 811 & 814 & D-C-C-D wagging \\
& 692 & 672 & D-C-C-D twisting \\
& 583 & 567 & D-C-C-D scissoring \\
\hline
\end{tabular}

\subsection{Wavelength Dependent Product Distribution}

\section{Photoirradiation of $\mathrm{AZ}$ at $172 \mathrm{~nm}$}

Adam and coworkers studied solution phase denitrogenation of azoalkanes using a $185 \mathrm{~nm}$ light source. ${ }^{9}$ They proposed that $\pi-\pi^{*}$ excitation is facilitated by $185 \mathrm{~nm}$ irradiation, compared to $\mathrm{n}-\pi^{*}$ excitation at $350 \mathrm{~nm}$. They demonstrated that some azoalkanes show wavelength dependent photoproduct formation. ${ }^{10}$ To understand and characterize wavelength dependent product formation during photolysis of $\mathbf{A Z}$, Ar matrix isolated $\mathbf{A Z}$ was irradiated using a $172 \mathrm{~nm}$ light source for $20 \mathrm{~min}$ at $7 \mathrm{~K}$. This experiment resulted in the formation of $\mathbf{C P}$ (characterized as in Figure 8) and new peaks which were not assignable to $\mathbf{C P}$. The new peaks at 908 and $700 \mathrm{~cm}^{-1}$ were in good agreement with those calculated (scaling factor $=0.9613)$ as the major peaks of cyclopentene (3) at $891(\mathrm{H}-\mathrm{C}=\mathrm{C}-\mathrm{H}$ rocking, $\mathrm{i}=5.2)$ and $690 \mathrm{~cm}^{-1}(\mathrm{H}-\mathrm{C}=\mathrm{C}-\mathrm{H}$ wagging, $\mathrm{i}=25.3)$ (Figures $\mathrm{S} 15 \mathrm{~B}$ and $\mathrm{D}$, Scheme $\mathrm{S} 1$ ).

\section{Photoirradiation of AZ at 310-350 nm}

Ar matrix containing $\mathbf{A Z}$ was irradiated at different wavelengths using a Xe lamp with a 310-350 $\mathrm{nm}$ filter. After $18 \mathrm{~h}$ photolysis at $7 \mathrm{~K}$, IR spectra showed $\mathbf{C P}$ to be the sole photoproduct (Figures S15C and E, Scheme S1); no peaks corresponding to the formation of $\mathbf{3}$ were observed. The IR spectra were consistent with that of irradiation by a high-pressure $\mathrm{Hg}$ lamp, with predominating formation of $\mathbf{C P}$ (Figure 8), versus photolysis at $7 \mathrm{~K}$ using the $172 \mathrm{~nm}$ light source yielding both $\mathbf{C P}$ and 3. Therefore, the product distribution of the photolysis of $\mathbf{A Z}$ was determined to be wavelength dependent.

Figure S15. IR spectra of: (A) AZ in Ar matrix at $20 \mathrm{~K}$; (B) difference between (A) and 20 min photolysis using a $172 \mathrm{~nm}$ light source at $7 \mathrm{~K}$; and (C) difference between (A) and $18 \mathrm{~h}$ photolysis using a Xe lamp with a $310-350 \mathrm{~nm}$ filter at $7 \mathrm{~K}$. Calculated IR spectra (scaling factor $=0.9613$ ) of: (D) 3 and (E) CP at the B3LYP/6-31G(d) level of theory. 


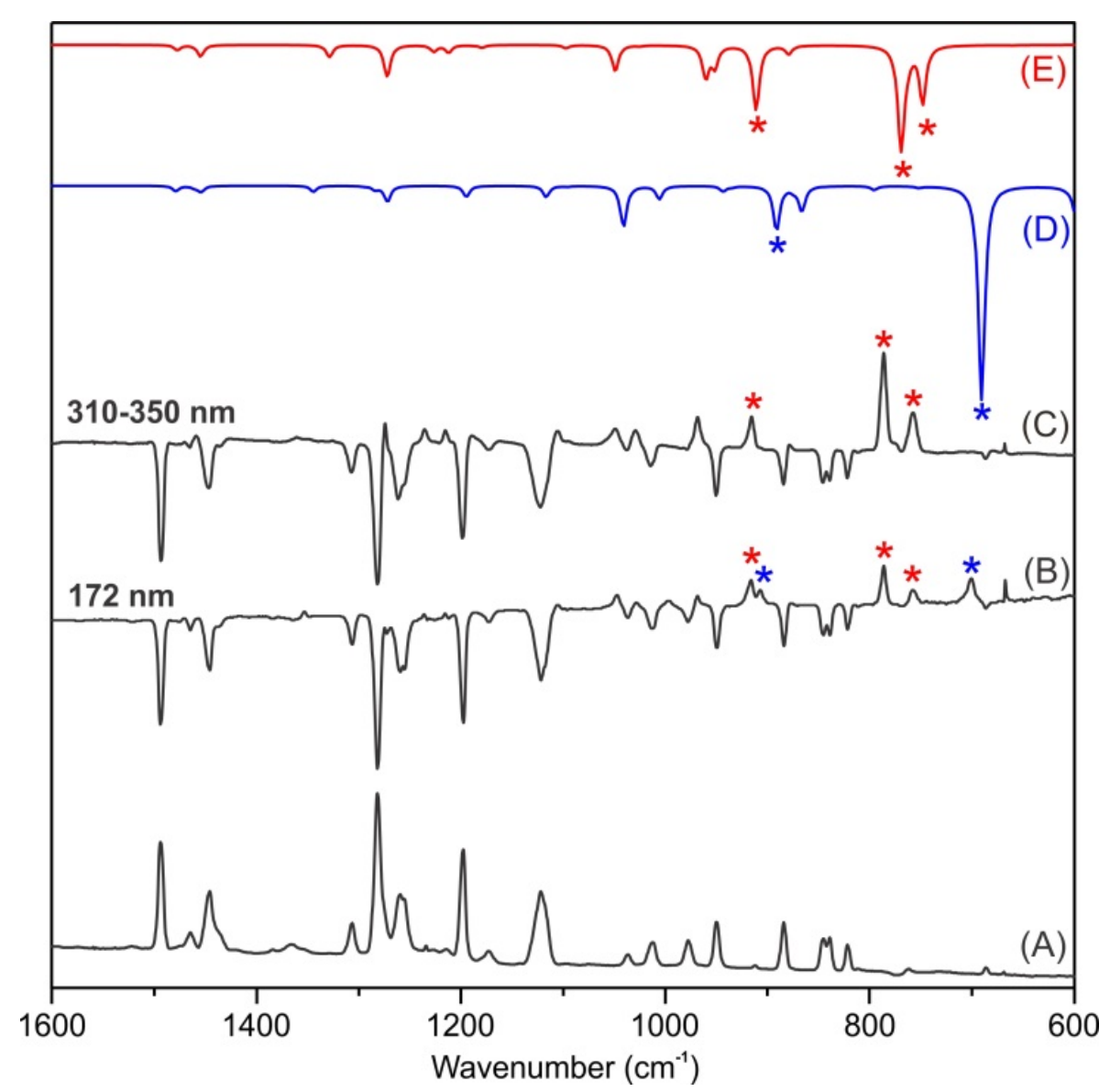

Scheme S1. Wavelength-dependent selectivity in the photolysis of 2,3-diazabicyclo[2.2.1]hept-2-ene (AZ).

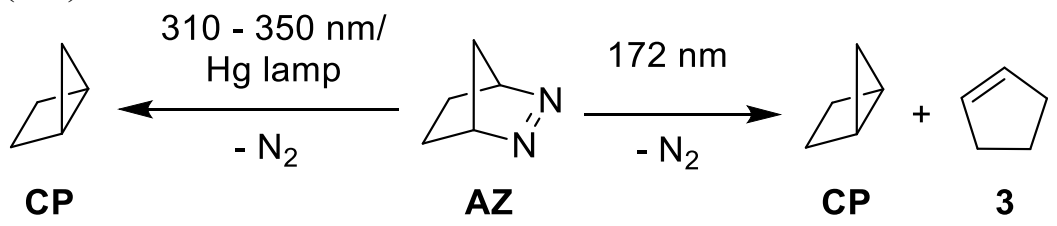

\subsection{Photoproducts' Stereoselectivity at Room Temperature in Solution (Characterization of CP)}

In $\mathbf{C P}$, the bridgehead proton $\mathrm{H}_{\mathrm{b}}(\delta=1.44 \mathrm{ppm})$ is coupled with $\mathrm{H}_{\text {exo }}$, but not with $\mathrm{H}_{\text {endo, }}$, because of the dihedral angle of $\approx 90^{\circ}$ between $\mathrm{H}_{\mathrm{b}}$ and $\mathrm{H}_{\text {endo }}$ (Figure S16). Thus, the signals at $\delta=1.97$ and 1.29 ppm were assigned to inv-CP- $\mathrm{d}_{6}\left(\mathrm{H}_{\text {exo }}\right)$ and ret- $\mathbf{C P}-\mathrm{d}_{6}\left(\mathrm{H}_{\text {endo }}\right)$, respectively. The assignments were also supported by NMR computations of inv-CP- $\mathrm{d}_{6}$ and ret-CP- $\mathrm{d}_{6}$ at the B3LYP/6-31G(d) level of theory using the gauge-independent atomic orbital (GIAO) method. Using tetramethylsilane (TMS) as a reference, the calculated ${ }^{1} \mathrm{H}-\mathrm{NMR}$ spectra of inv-CP- $\mathrm{d}_{6}$ and ret-CP- $\mathrm{d}_{6}$ had peaks at $\delta=2.44$ and $1.83 \mathrm{ppm}$, respectively. Through comparisons with Figure 9, the higher experimental peak (1.97 ppm) was assigned to inv-CP- $\mathrm{d}_{6}$, while the lower peak $(1.29 \mathrm{ppm})$ was assigned to ret-CP- $\mathrm{d}_{6}$, in a ratio of 67:33 (Table 2, entries 1, 2).

Figure S16. Bridge-head, exo, and endo protons in $\mathbf{C P}$. 


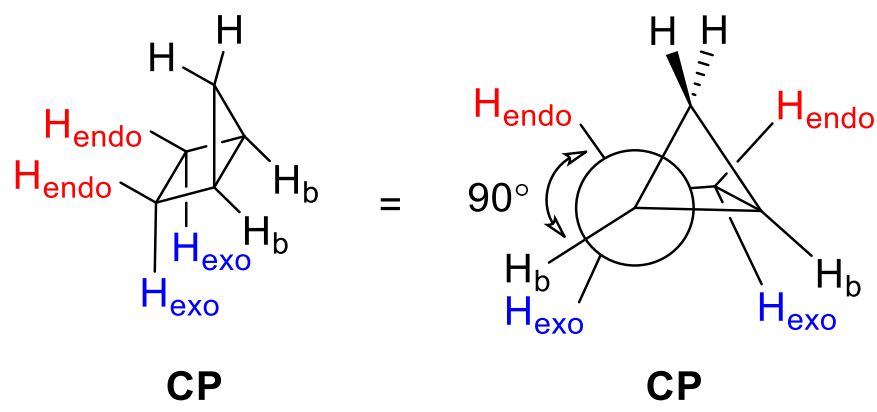

Figure S17. ${ }^{1} \mathrm{H}-\mathrm{NMR}\left(400 \mathrm{MHz}, \mathrm{CDCl}_{3}\right),{ }^{13} \mathrm{C}\left\{{ }^{1} \mathrm{H}\right\}$-NMR (100 MHz, $\mathrm{CDCl}_{3}$ ), and COSY-NMR spectra of $\mathbf{C P}$.

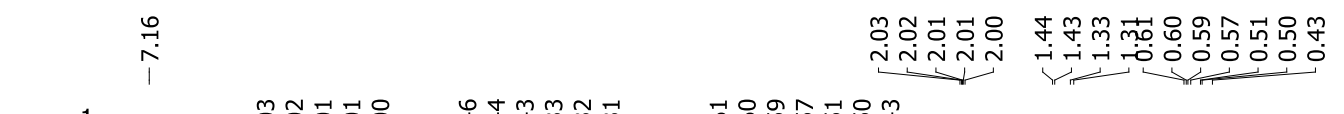

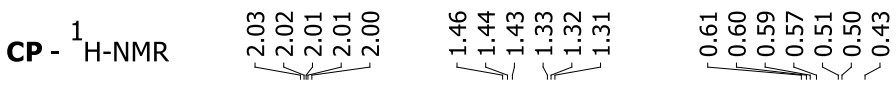
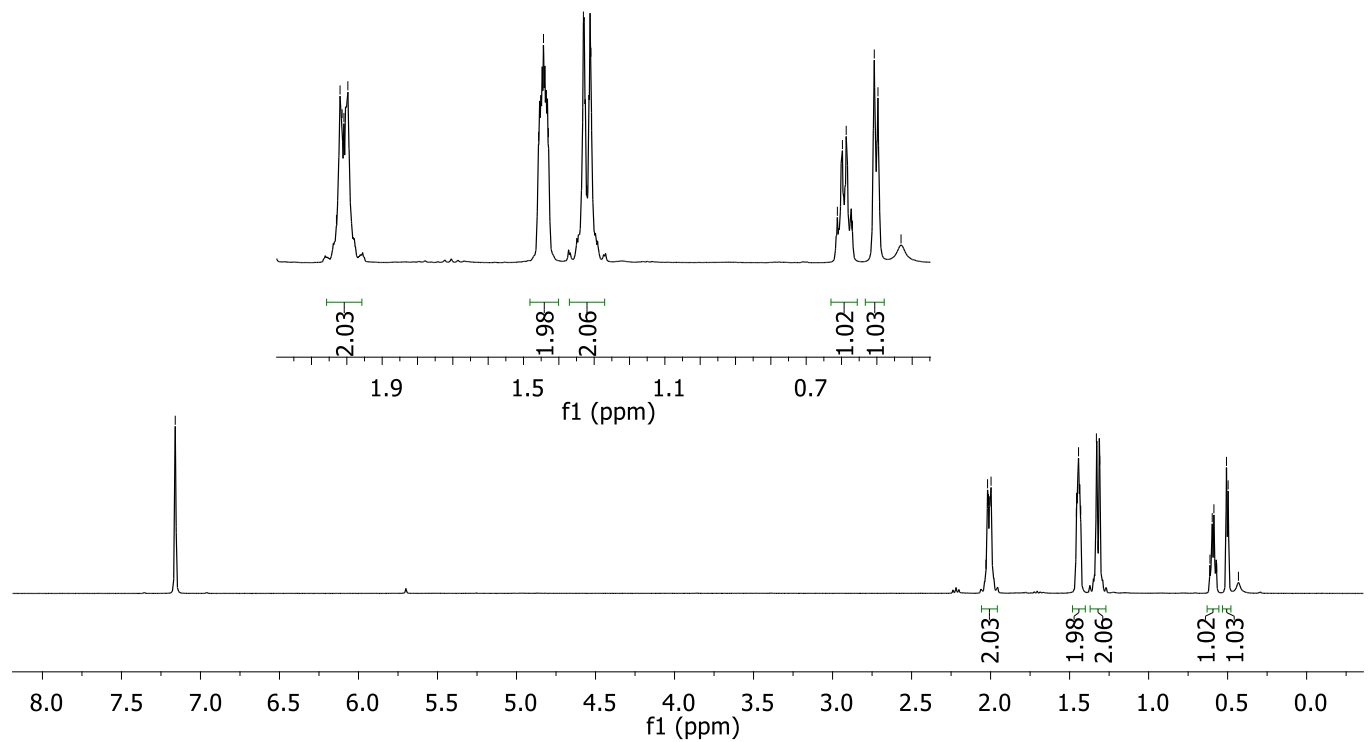


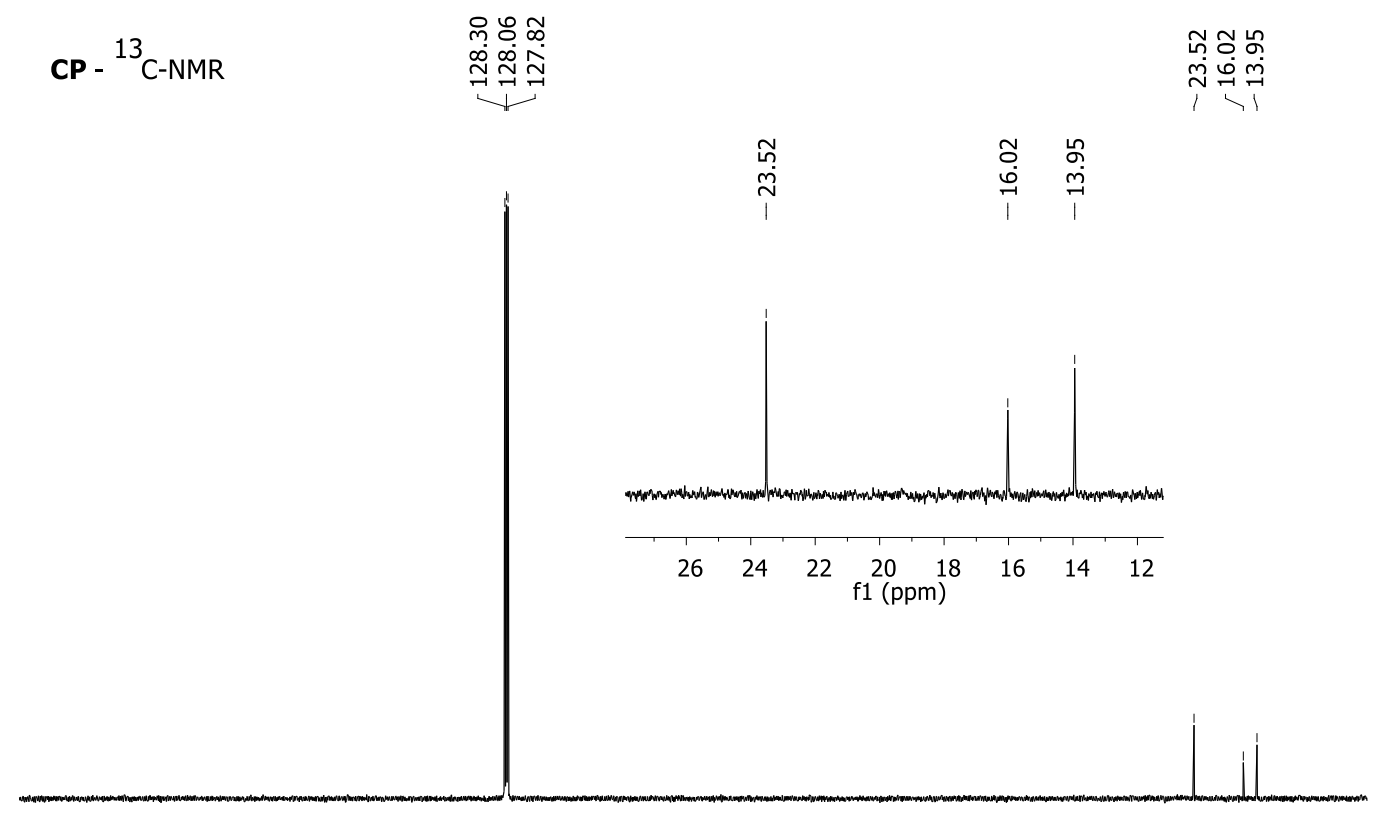

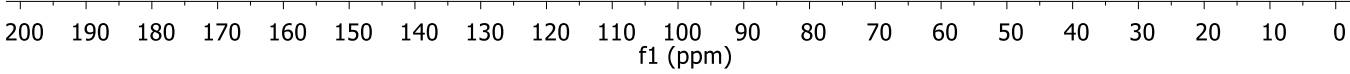

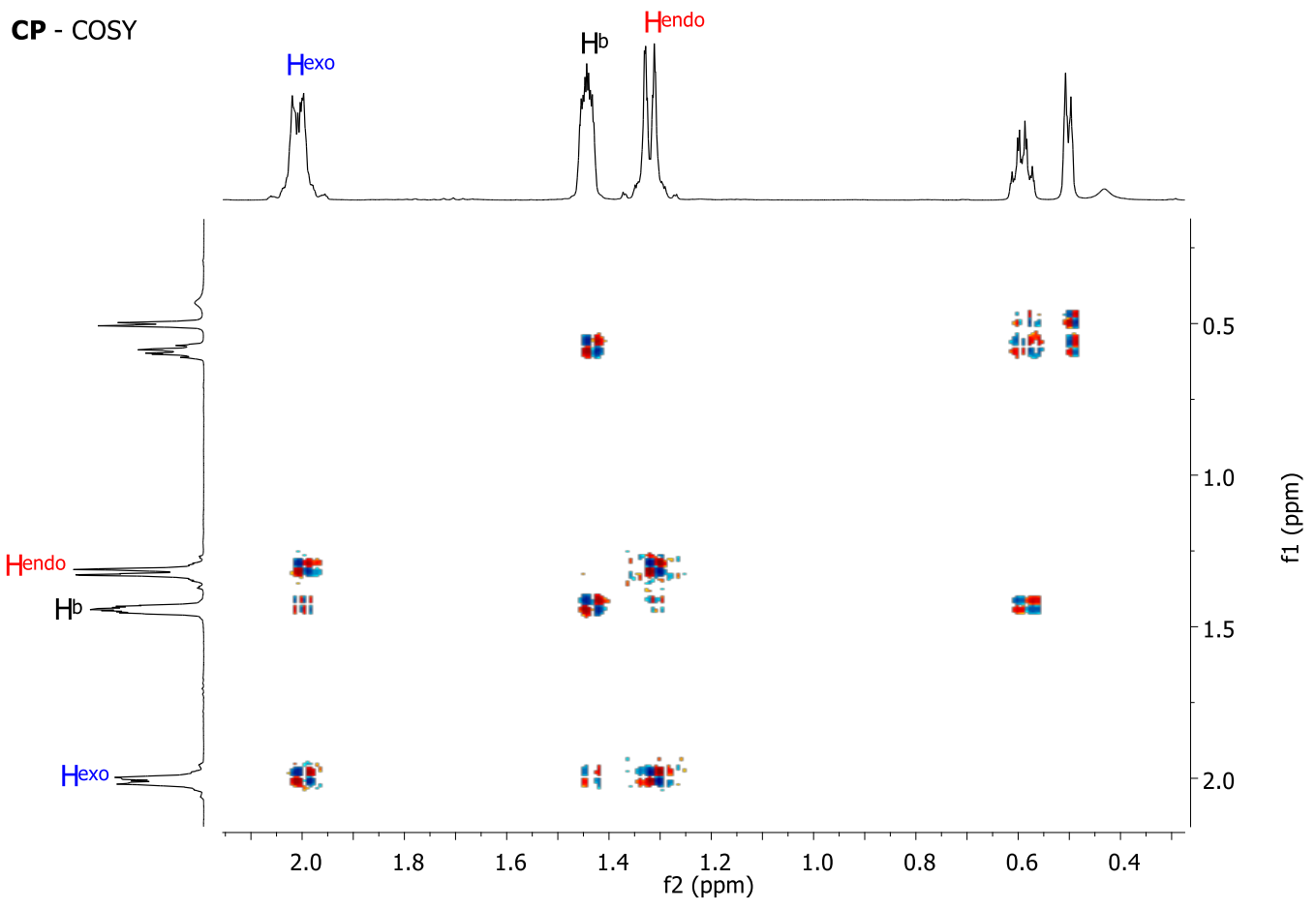




\subsection{Photoproducts' Stereoselectivity in Low-Temperature Photolysis in Ar Matrix}

Figure S18. Photolysis of AZ- $\mathrm{d}_{6}$ in solid state monitored using ${ }^{1} \mathrm{H}-\mathrm{NMR}$ (400 $\mathrm{MHz}, \mathrm{C}_{6} \mathrm{D}_{6}$ ) spectroscopy after dissolving the photolyzed sample mixture in $\mathrm{C}_{6} \mathrm{D}_{6}$.
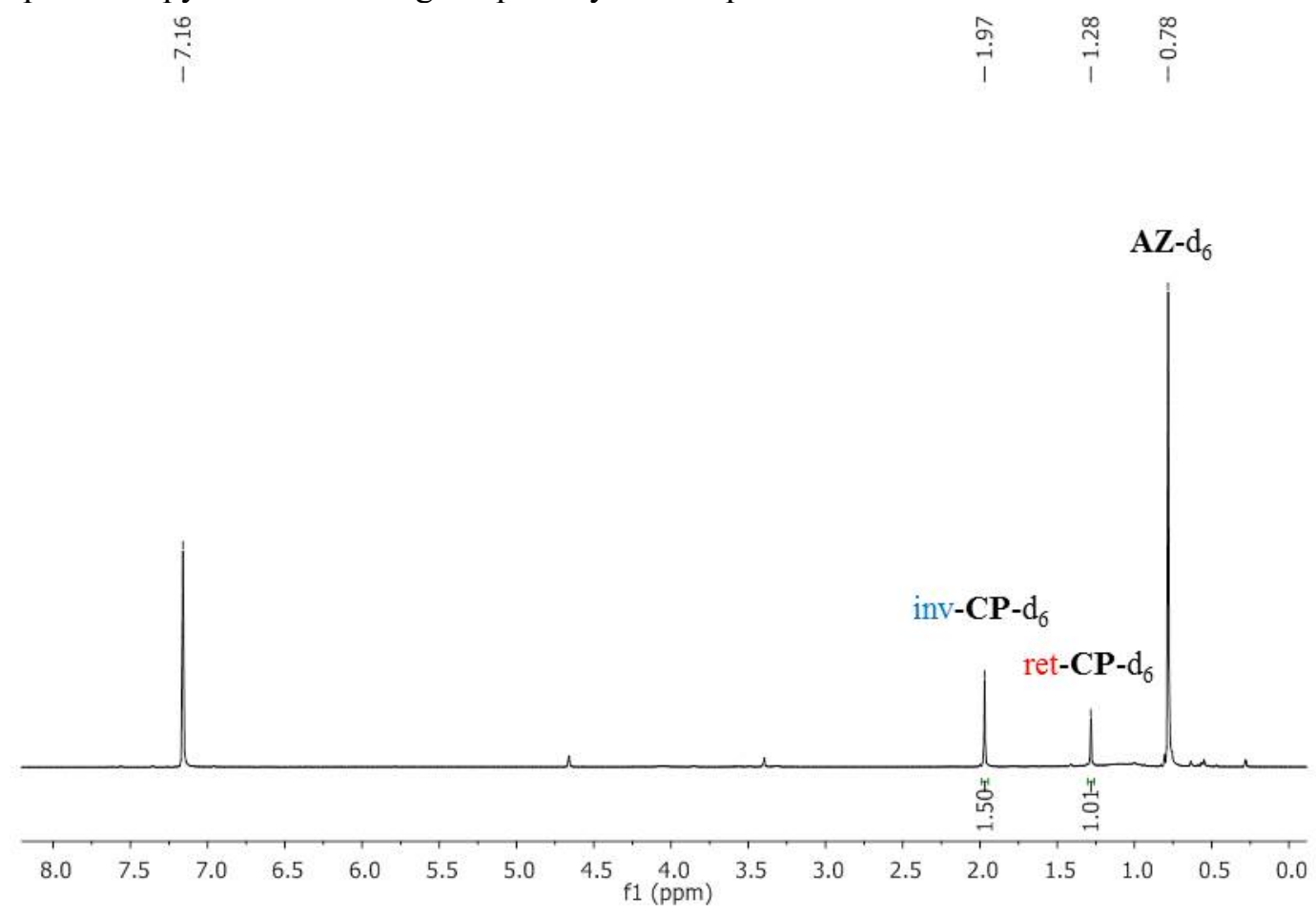

\subsection{Spin State and Temperature Effects on Stereoselectivity in Solution}

Figure S19. Photolysis of AZ- $\mathrm{d}_{6}$ in presence of 10 equivalents benzophenone (BP) photosensitizer in $\mathrm{C}_{6} \mathrm{D}_{6}$ solvent using $365 \mathrm{~nm}$ LED lamp and monitored using ${ }^{1} \mathrm{H}-\mathrm{NMR}$ (400 MHz, $\mathrm{C}_{6} \mathrm{D}_{6}$ ) spectroscopy. Bottom: 0 min photolysis and top: 2 min photolysis.
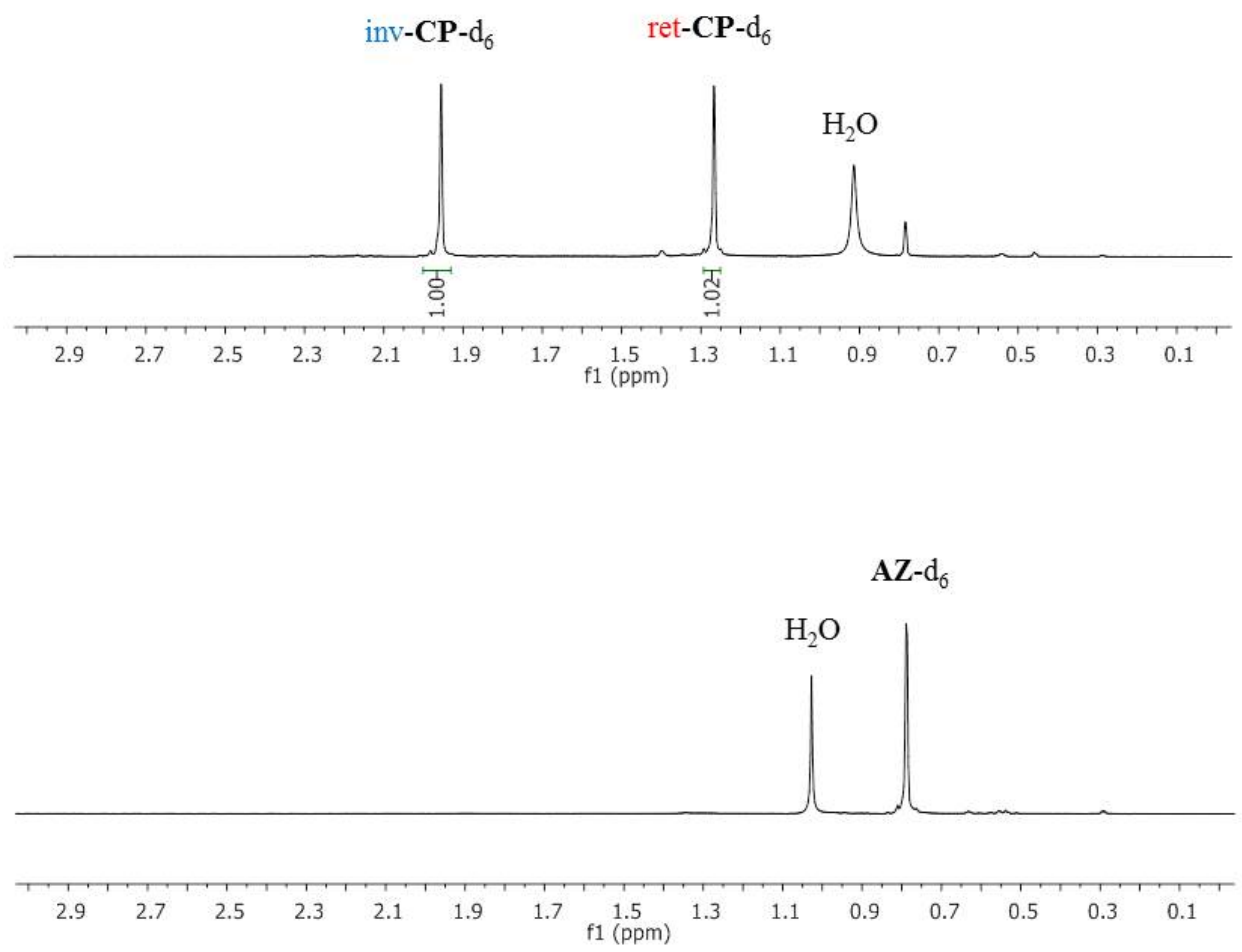
Figure S20. Photolysis of AZ- $\mathrm{d}_{6}$ at $-80{ }^{\circ} \mathrm{C}$ (bottom) and $+80{ }^{\circ} \mathrm{C}$ (top) in toluene- $\mathrm{d}_{8}$ solvent using a high-pressure $\mathrm{Hg}$ lamp and monitored using ${ }^{1} \mathrm{H}-\mathrm{NMR}\left(400 \mathrm{MHz}\right.$, Toluene- $\left.\mathrm{d}_{8}\right)$ spectroscopy.

$+80{ }^{\circ} \mathrm{C}$

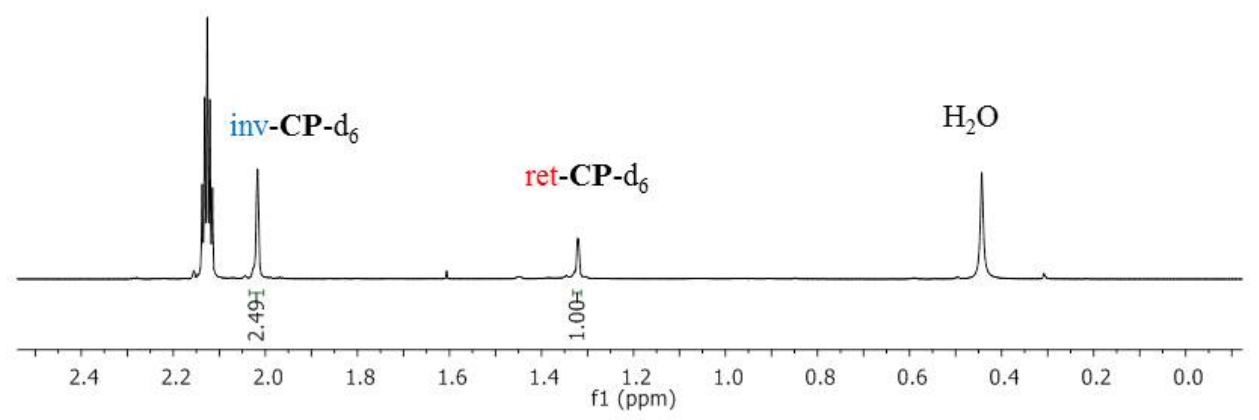

$-80{ }^{\circ} \mathrm{C}$

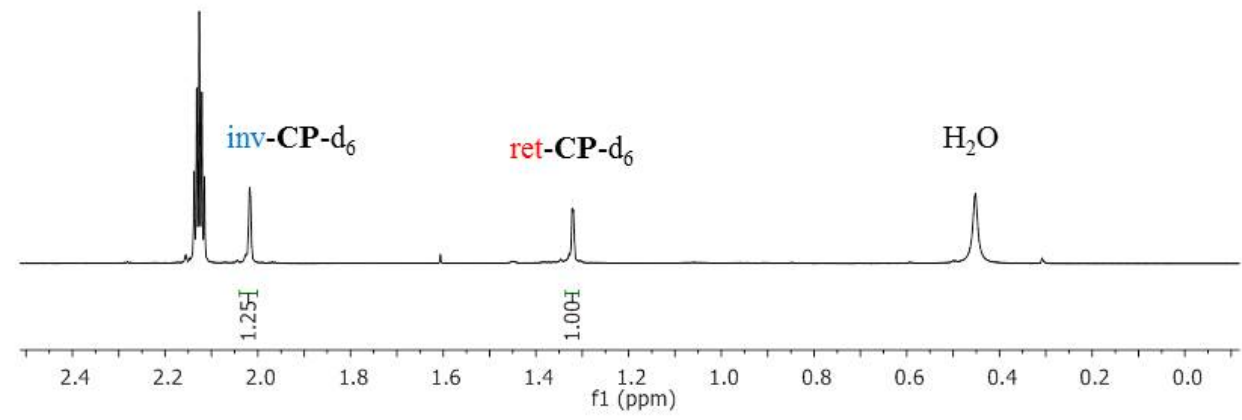

Figure S21. Comparison of ${ }^{1} \mathrm{H}-\mathrm{NMR}\left(400 \mathrm{MHz}, \mathrm{CDCl}_{3}\right.$ ) spectra of $\mathbf{A Z}$ (bottom) and $\mathbf{A Z}-\mathrm{d}_{6}$ (top) in $\mathrm{CDCl}_{3}$ solvent.

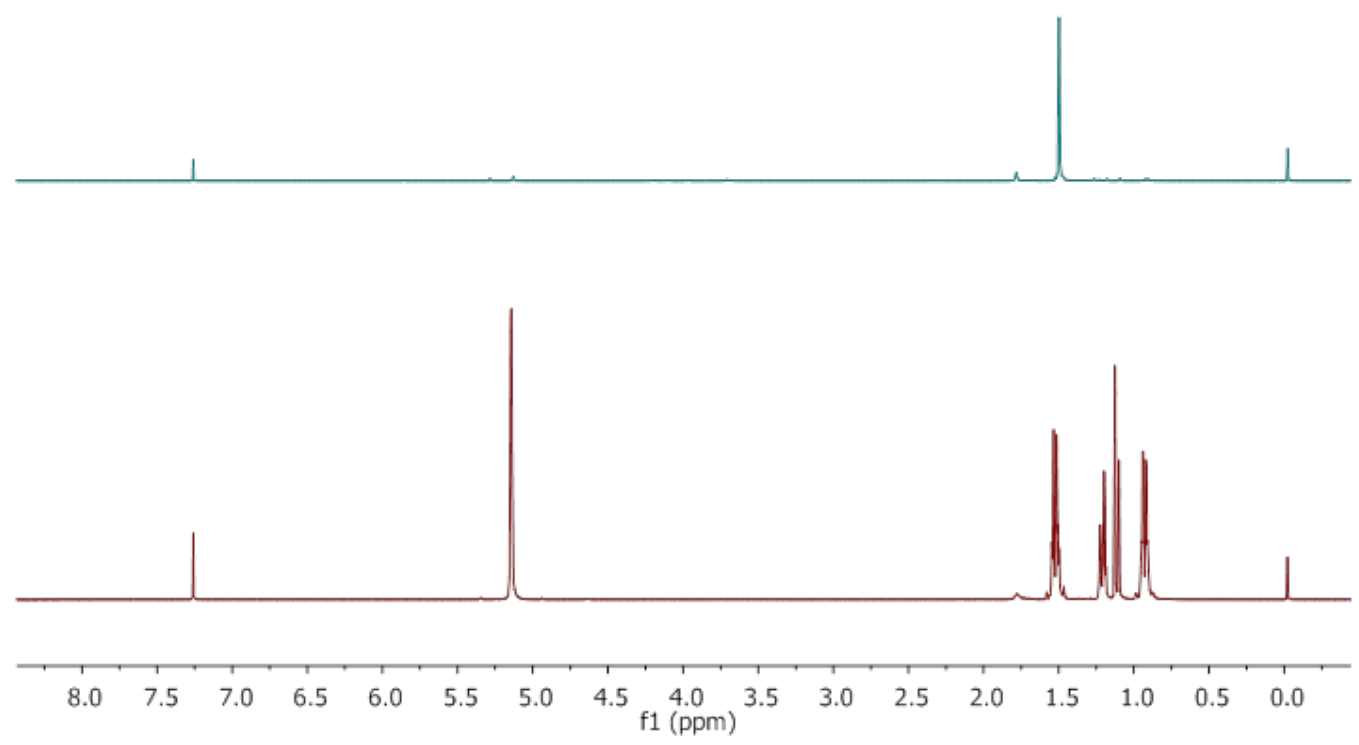




\subsection{Synthesis}

Scheme S2. Synthesis process of AZ.

$\triangle+\stackrel{\mathrm{N}}{\mathrm{N}}_{\mathrm{NO}_{2} \mathrm{Et}}^{\mathrm{N}^{-\mathrm{CO}_{2} \mathrm{Et}}} \stackrel{\text { dry Et}}{2} \mathrm{O}$<smiles>CCOC(=O)N1C2C=CC(C2)N1C(=O)OCC</smiles>

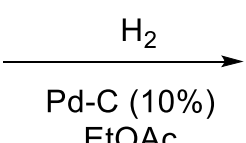<smiles>CCOC(=O)N1C2CCC(C2)N1C(=O)OCC</smiles><smiles>C1CC2CC1N=N2</smiles><smiles>[GeH3]</smiles>
$A Z$

Cracking of dicyclopentadiene (dimer) was done using a fractional distillation setup to break down the dimer into monomer (cyclopentadiene).

Figure S22. ${ }^{1} \mathrm{H}-\mathrm{NMR}\left(400 \mathrm{MHz}, \mathrm{CDCl}_{3}\right)$ and ${ }^{13} \mathrm{C}\left\{{ }^{1} \mathrm{H}\right\}-\mathrm{NMR}\left(100 \mathrm{MHz}, \mathrm{CDCl}_{3}\right)$ spectra of diethyl2,3-diazabicyclo[2.2.1] hept-5-ene-2,3-dicarboxylate

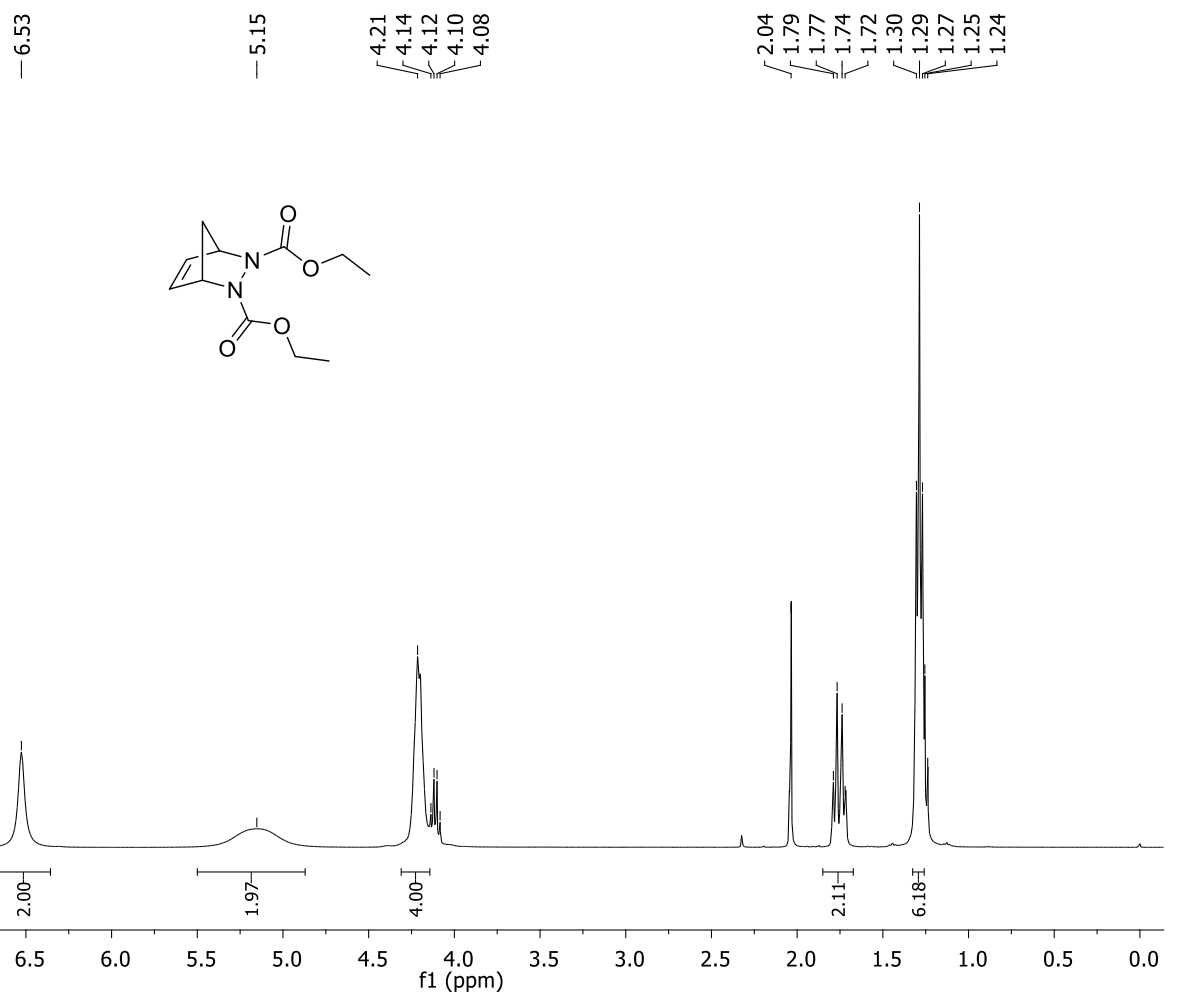




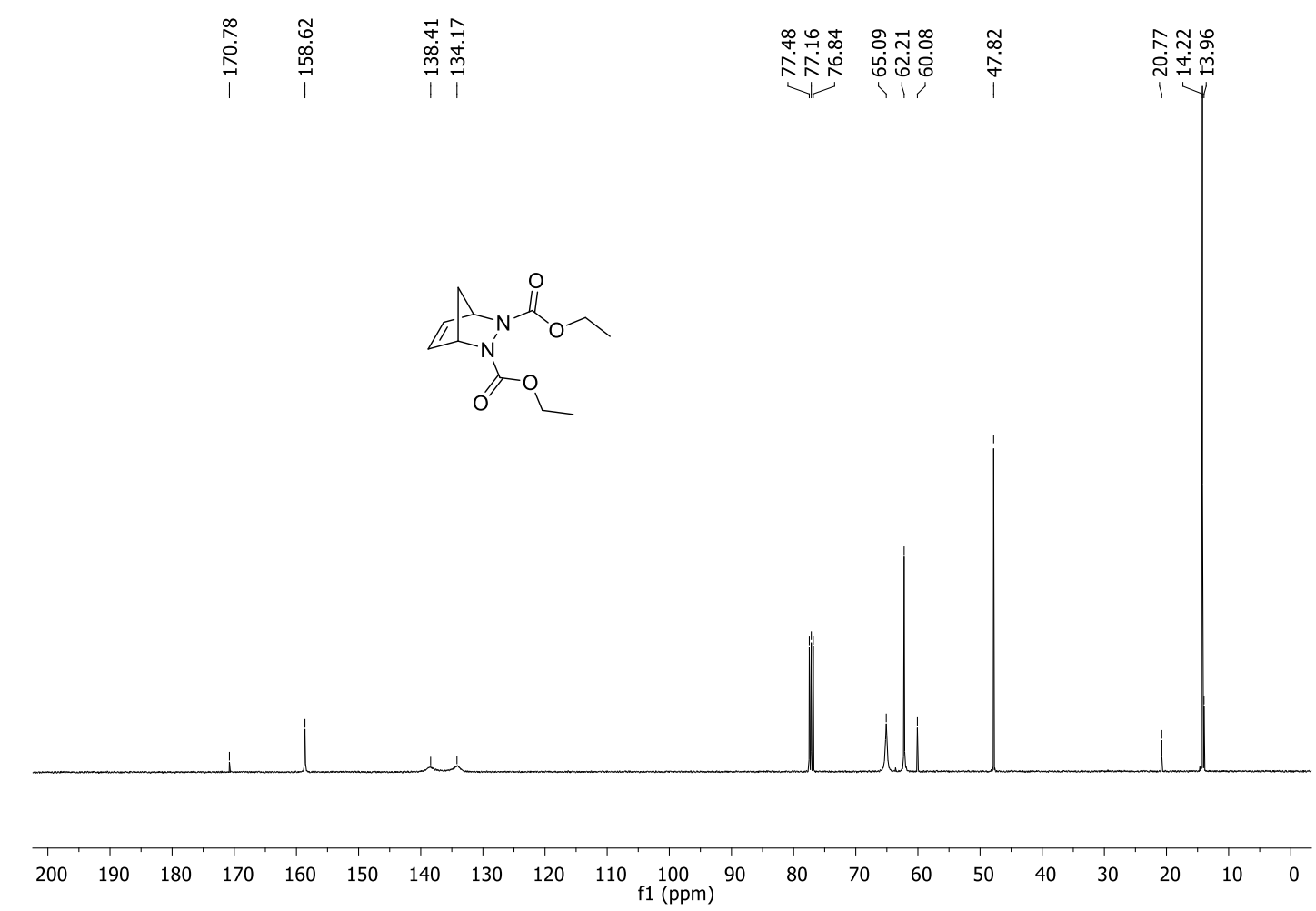

Figure S23. ${ }^{1} \mathrm{H}-\mathrm{NMR}\left(400 \mathrm{MHz}, \mathrm{CDCl}_{3}\right)$ and ${ }^{13} \mathrm{C}\left\{{ }^{1} \mathrm{H}\right\}-\mathrm{NMR}\left(100 \mathrm{MHz}, \mathrm{CDCl}_{3}\right)$ spectra of diethyl2,3-diazabicyclo[2.2.1] heptane-2,3-dicarboxylate

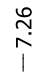

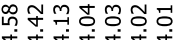

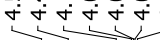

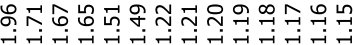
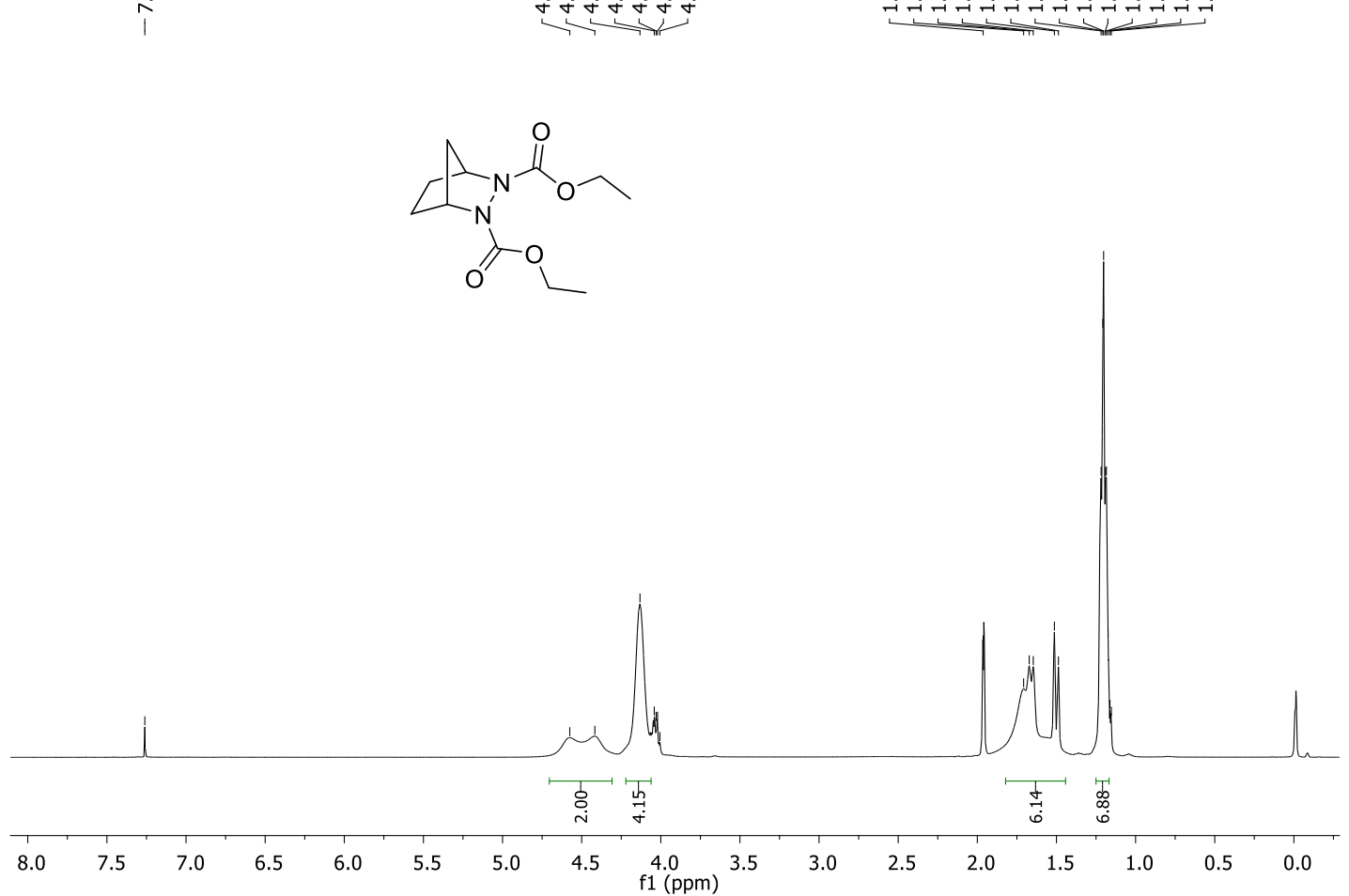

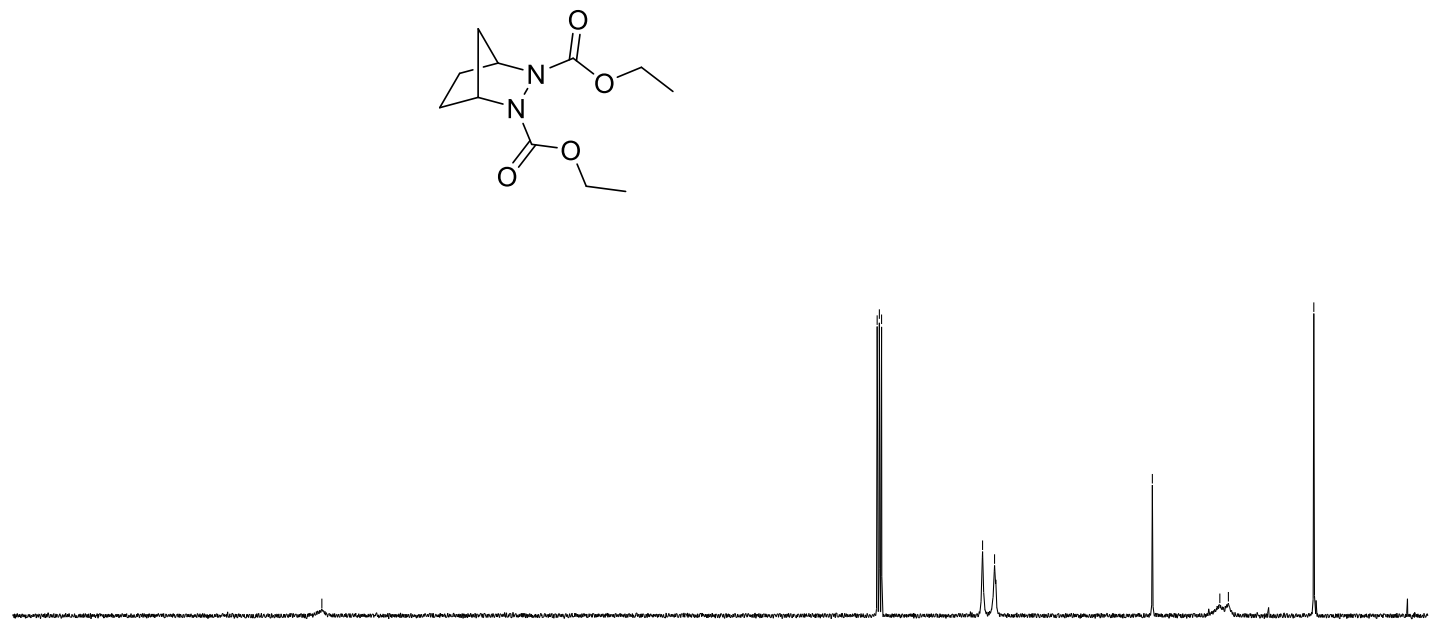

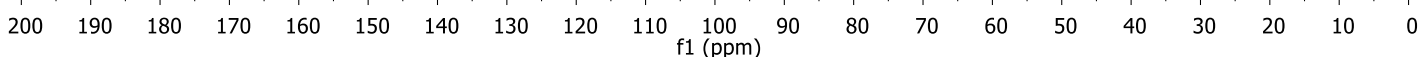

Figure S24. ${ }^{1} \mathrm{H}-\mathrm{NMR}\left(400 \mathrm{MHz}, \mathrm{CDCl}_{3}\right),{ }^{13} \mathrm{C}\left\{{ }^{1} \mathrm{H}\right\}$-NMR $\left(100 \mathrm{MHz}, \mathrm{CDCl}_{3}\right)$, and DEPT $135\left\{{ }^{1} \mathrm{H}\right\}$ NMR (100 MHz, $\mathrm{CDCl}_{3}$ ), and IR spectra of 2,3-diazabicyclo[2.2.1]hept-2-ene (AZ)

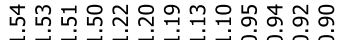<smiles>C1CC2CC1N=N2</smiles>

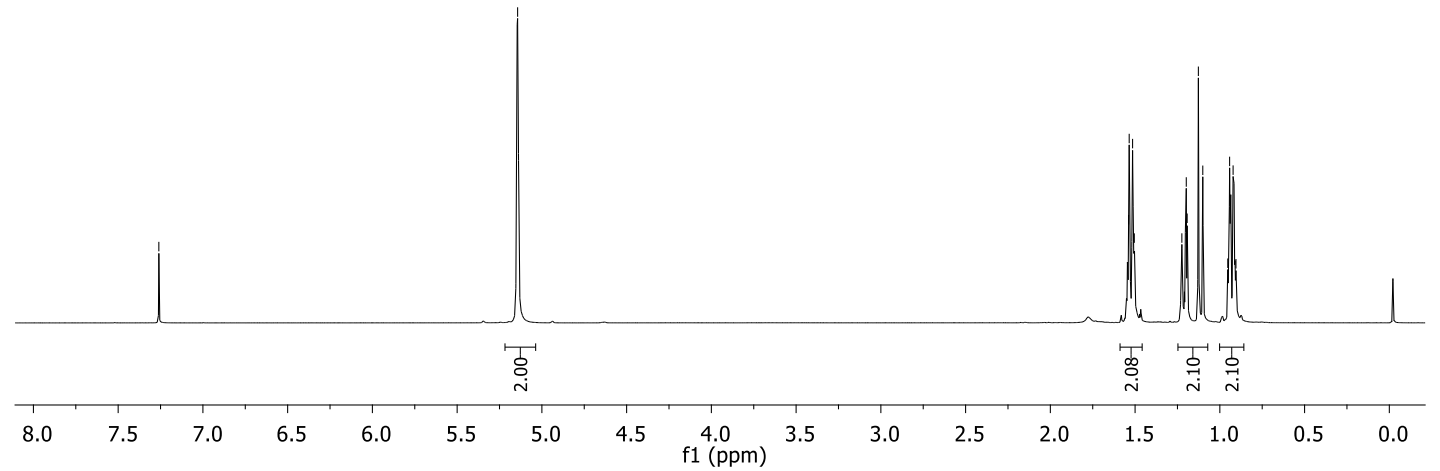




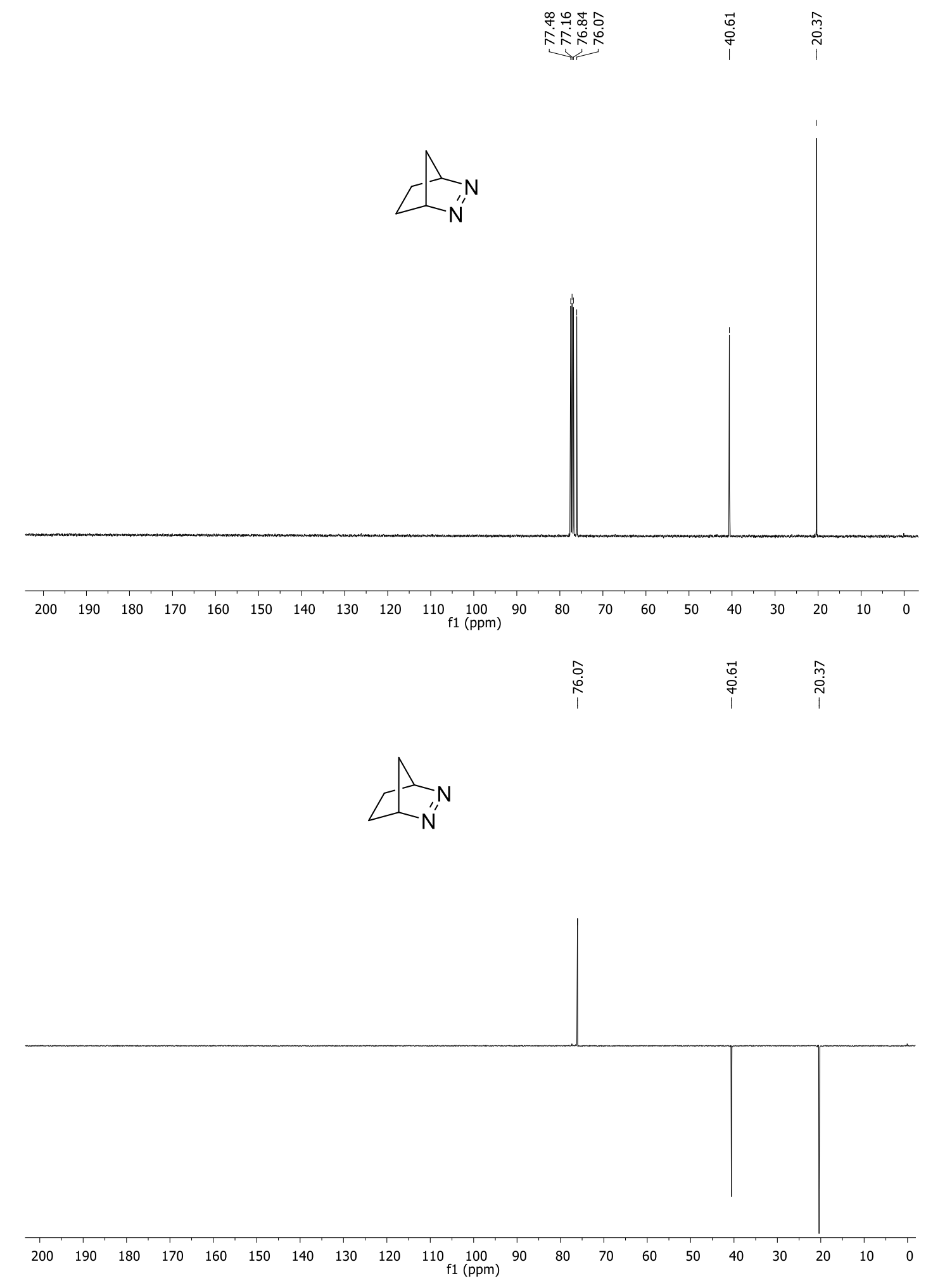




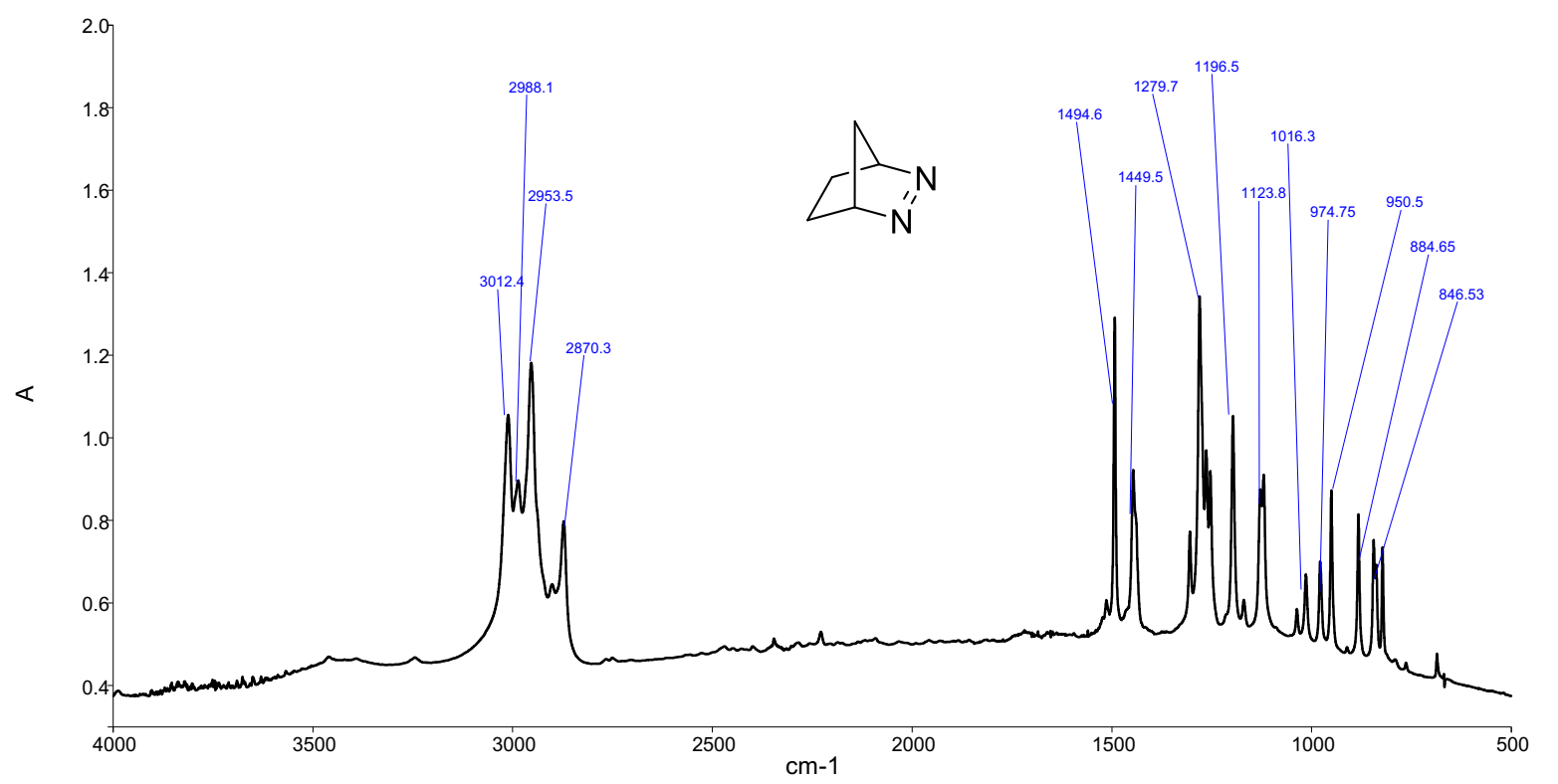

Figure S25. ${ }^{1} \mathrm{H}-\mathrm{NMR}\left(400 \mathrm{MHz}, \mathrm{CDCl}_{3}\right)$ and ${ }^{13} \mathrm{C}\left\{{ }^{1} \mathrm{H}\right\}-\mathrm{NMR}\left(100 \mathrm{MHz}, \mathrm{CDCl}_{3}\right)$ spectra, and HRMS (ESI) of AZ-d 6

$\stackrel{\stackrel{0}{i}}{i}$
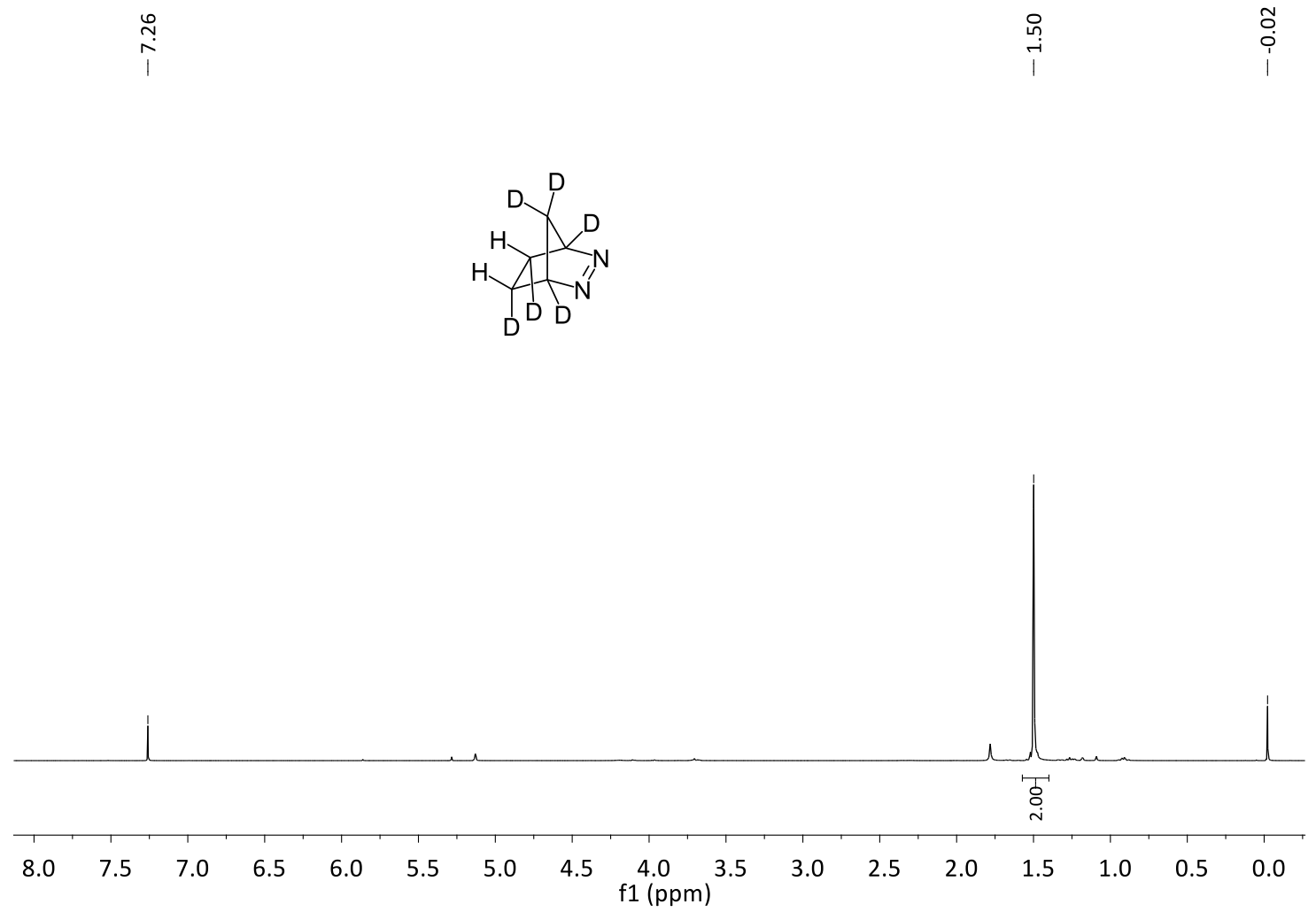


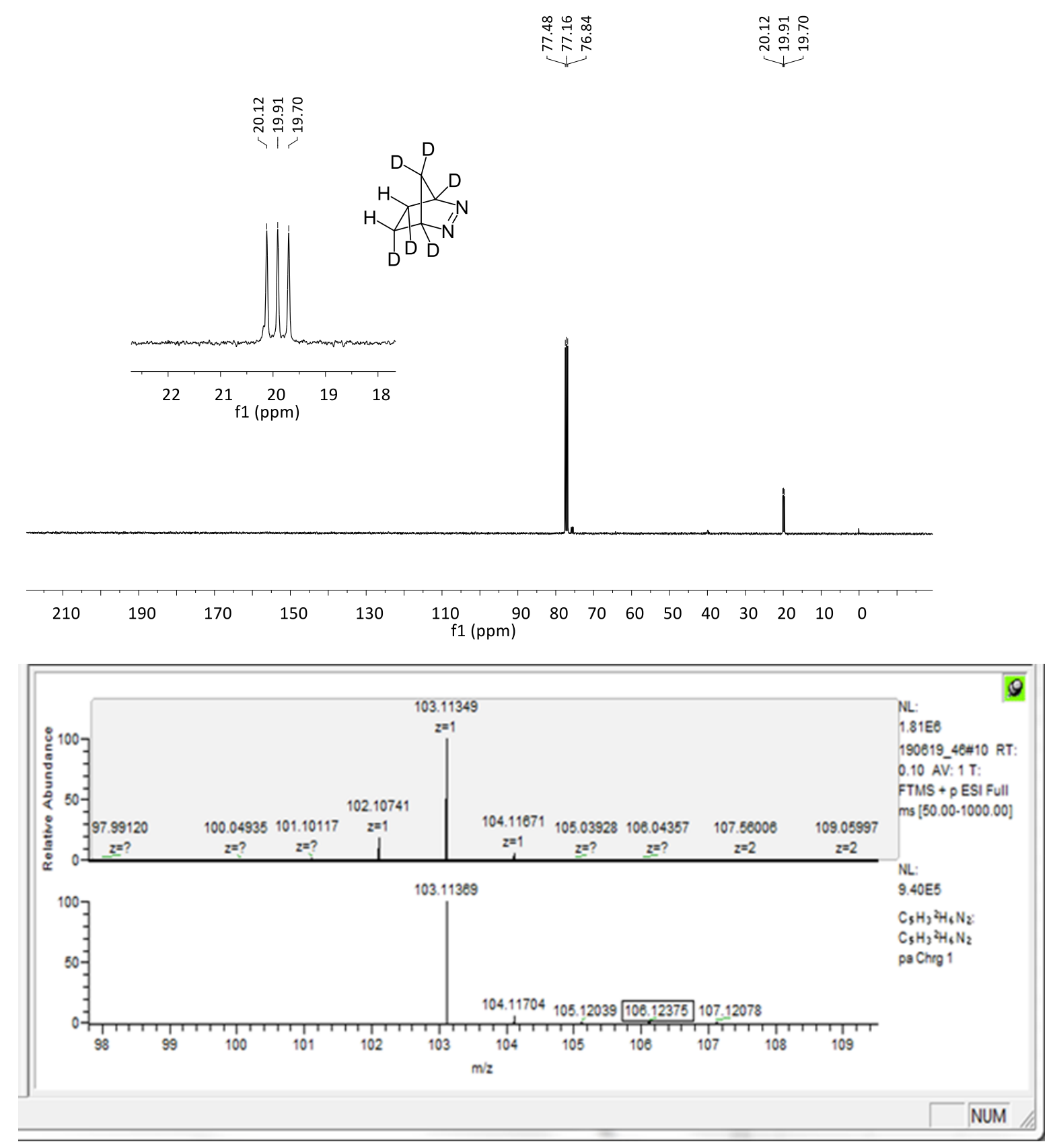

\section{Quantum Chemical Calculation}

The obtained values of $\mathrm{S}_{1}$ and $\mathrm{T}_{1}$ of $\mathbf{A Z}$ were 82.89 and $58.84 \mathrm{kcal} \mathrm{mol}^{-1}$ over $\mathrm{S}_{0}$, respectively. TD-DFT gave a value of $84.37 \mathrm{kcal} \mathrm{mol}^{-1}$ for the vertical excitation from $\mathrm{S}_{0}$ of $\mathbf{A Z}$ to $\mathrm{S}_{1}$ of $\mathbf{A Z}$. In the literature, a fluorescence spectrum ${ }^{11}$ of $\mathbf{A Z}$ in gas phase showed a 0 '-0" band at $84.5 \mathrm{kcal} \mathrm{mol}^{-1}$, and a phosphorescence spectrum $^{12}$ of $\mathbf{A Z}$ in EPA (ether:isopentane:alcohol $=5: 5: 2 \mathrm{v} / \mathrm{v}$ ) showed the triplet energy to be $62 \mathrm{kcal} \mathrm{mol}^{-1}$. Thus, the computations reproduced the experimental observations. In the triplet state $\mathrm{T}_{1}$ of $\mathbf{A Z}$, the $\mathrm{N}=\mathrm{N}$ bond was elongated compared to $\mathrm{S}_{0}(1.31$ vs. $1.25 \AA)$ and spin densities were located primarily on the two nitrogen atoms (0.91 and 0.84) (Figure 13, Table S3). The optimized T-1 structure, which is formed after the first $\mathrm{C}-\mathrm{N}$ bond cleavage, showed a much shorter $\mathrm{N}=\mathrm{N}$ bond $(1.17 \AA)$. 


\subsection{Alternative Reactions}

Hydrogen atoms were detected in the EPR spectra in MTHF, 3MP, and Ar matrices at cryogenic temperatures using a high-pressure $\mathrm{Hg}$ lamp (> $250 \mathrm{~nm}$ ). TD-DFT calculations of T-DR detected all electronic transitions at $\lambda<200 \mathrm{~nm}$, with the highest wavelength at $195 \mathrm{~nm}$ (Figure S33). Therefore, it is unlikely that T-DR absorbed light to yield cyclopentene 3. However, TD-DFT calculations of S-DR showed main absorptions at $\lambda>200 \mathrm{~nm}$, occurring at 429 and $410 \mathrm{~nm}$ (Figure S35). Therefore, light can be absorbed by S-DR to result in the secondary photolysis and produce $\mathbf{3}$ via cyclopentene radical (2) and hydrogen atom pair (Scheme 4). During $172 \mathrm{~nm}$ photolysis, photochemistry can also occur from higher excited singlet states of $\mathbf{A Z}\left(\mathrm{S}_{2}=145 \mathrm{kcal} \mathrm{mol}^{-1}\right.$, TD-DFT) to form $\mathbf{3}$. Furthermore, there is a possibility that $\mathrm{S}-\mathbf{1}$ yields the radical pair that combines to form $\mathbf{3}$.

\subsection{Optimized Structures}

Figure S26. Optimization of AZ.

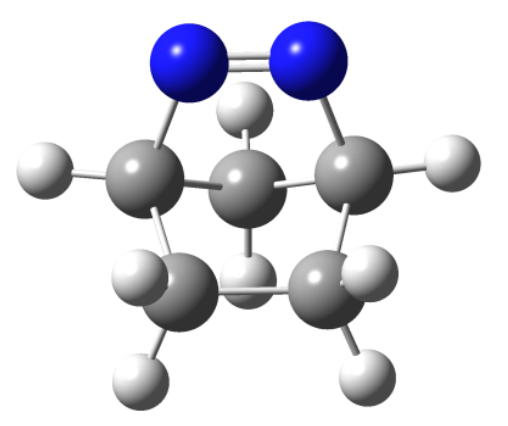

DFT/B3LYP 6-31G(d)

$\mathrm{E}=-304.79347541$ a.u.

Zero-point correction $=0.129978$ a.u.

Standard orientation:

\begin{tabular}{|c|c|c|c|c|}
\hline Center & Atomic & Atomic & Coordinat & tes (Angstroms) \\
\hline Number & Numbe & Tyре & $\mathrm{X}$ & $\mathrm{Z}$ \\
\hline 1 & 6 & 1.226108 & 0.778532 & -0.328232 \\
\hline 2 & 6 & -0.167657 & 1.098865 & 0.289032 \\
\hline 3 & 6 & -0.168409 & -1.098699 & 0.289133 \\
\hline 4 & 6 & 1.225696 & -0.779136 & -0.328093 \\
\hline 5 & 1 & 1.323868 & 1.208374 & -1.328582 \\
\hline 6 & 1 & 2.023364 & 1.191194 & 0.297863 \\
\hline 7 & 1 & 1.323392 & -1.209240 & -1.328352 \\
\hline 8 & 1 & 2.022540 & -1.192133 & 0.298257 \\
\hline 9 & 6 & -0.320037 & 0.000259 & 1.348775 \\
\hline
\end{tabular}




$\begin{array}{rrrrrr}10 & 1 & 0 & -1.299173 & 0.000637 & 1.837604 \\ 11 & 1 & 0 & 0.473899 & -0.000078 & 2.101313 \\ 12 & 1 & 0 & -0.348280 & 2.139425 & 0.557408 \\ 13 & 1 & 0 & -0.349246 & -2.139130 & 0.557917 \\ 14 & 7 & 0 & -1.139001 & -0.625312 & -0.758259 \\ 15 & 7 & 0 & -1.138794 & 0.625600 & -0.758473\end{array}$

Figure S27. TD-DFT calculation of AZ.

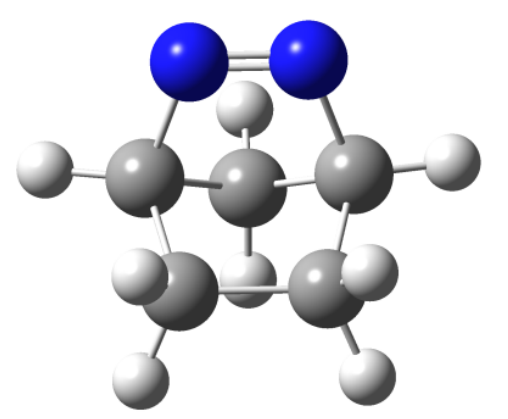

Excitation energies and oscillator strengths:

Excited State 1: $\quad$ Singlet-A $\quad 3.6580 \mathrm{eV} 338.94 \mathrm{~nm} \mathrm{f}=0.0004<\mathrm{S}^{* *} 2>=0.000$ $26->27 \quad 0.70762$

Excited State 2: $\quad$ Singlet-A $\quad 6.2913 \mathrm{eV} 197.07 \mathrm{~nm} \mathrm{f}=0.0000<\mathrm{S}^{* *} 2>=0.000$

$$
\begin{array}{ll}
21->27 & -0.12470 \\
24->27 & 0.65999 \\
25->27 & 0.21734
\end{array}
$$

Excited State 3: $\quad$ Singlet-A $\quad 7.0512 \mathrm{eV} 175.83 \mathrm{~nm} \mathrm{f}=0.0289<\mathrm{S}^{* *} 2>=0.000$

$$
\begin{array}{ll}
21->27 & -0.17512 \\
24->27 & -0.23598 \\
25->27 & 0.62276
\end{array}
$$

Excited State 4: $\quad$ Singlet-A $\quad 7.2582 \mathrm{eV} 170.82 \mathrm{~nm} \mathrm{f}=0.0059<\mathrm{S}^{* *} 2>=0.000$ $23->27 \quad 0.70060$

Excitation energies and oscillator strengths:

Excited State 1: Triplet-A $2.5563 \mathrm{eV} 485.02 \mathrm{~nm} \mathrm{f}=0.0000<\mathrm{S} * * 2>=2.000$

$$
26->27 \quad 0.70688
$$

Excited State 2: $\quad$ Triplet-A $\quad 4.3056 \mathrm{eV} 287.96 \mathrm{~nm} \mathrm{f}=0.0000<\mathrm{S}^{* *} 2>=2.000$

$$
\begin{array}{ll}
17->27 & -0.13872 \\
19->27 & -0.14121 \\
21->27 & 0.20685 \\
25->27 & 0.65149
\end{array}
$$


$25<-27 \quad 0.11024$

Excited State 3: $\quad$ Triplet-A $\quad 5.3105 \mathrm{eV} 233.47 \mathrm{~nm} \mathrm{f}=0.0000<\mathrm{S} * * 2>=2.000$

$$
\begin{array}{ll}
21->27 & -0.22586 \\
24->27 & 0.65872
\end{array}
$$

Figure S28. Optimization of $\mathrm{T}_{1}$ of $\mathbf{A Z}$.

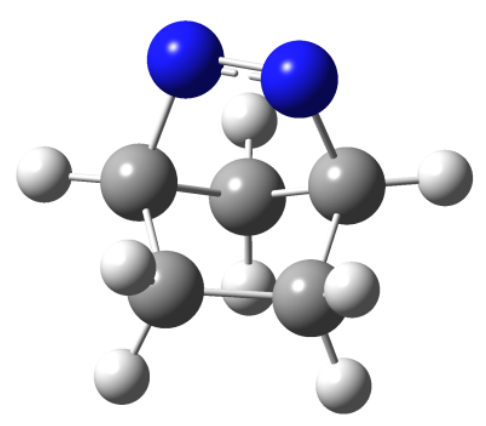

DFT/UB3LYP 6-31G(d)

$\mathrm{E}=-304.69971398$ a.u.

Zero-point correction $=0.127171$ a.u.

\begin{tabular}{|c|c|c|c|c|}
\hline Center & Atomic & Atomic & Coordinate & es (Angstroms) \\
\hline Number & Numbe & Type & $X$ & Z \\
\hline 1 & 6 & 1.072435 & 0.950416 & -0.385561 \\
\hline 2 & 6 & -0.358613 & 1.089658 & 0.185179 \\
\hline 3 & 6 & 0.018939 & -1.106155 & 0.329670 \\
\hline 4 & 6 & 1.377692 & -0.570981 & -0.196843 \\
\hline 5 & 1 & 1.111498 & 1.259674 & -1.433275 \\
\hline 6 & 1 & 1.768935 & 1.581067 & 0.177825 \\
\hline 7 & 1 & 1.672483 & -1.066642 & -1.123989 \\
\hline 8 & 1 & 2.158647 & -0.742111 & 0.556774 \\
\hline 9 & 6 & -0.376497 & 0.015264 & 1.320995 \\
\hline 10 & 1 & -1.369040 & -0.105405 & 1.759825 \\
\hline 11 & 1 & 0.367954 & 0.184011 & 2.107538 \\
\hline 12 & 1 & -0.702767 & 2.095526 & 0.423340 \\
\hline 13 & 1 & 0.004205 & -2.145089 & 0.660996 \\
\hline 14 & 7 & -0.848754 & -0.832059 & -0.870613 \\
\hline 15 & 7 & -1.353482 & 0.356310 & -0.650768 \\
\hline
\end{tabular}

Standard orientation: 
Table S3. Spin density of $T_{1}$ of AZ.

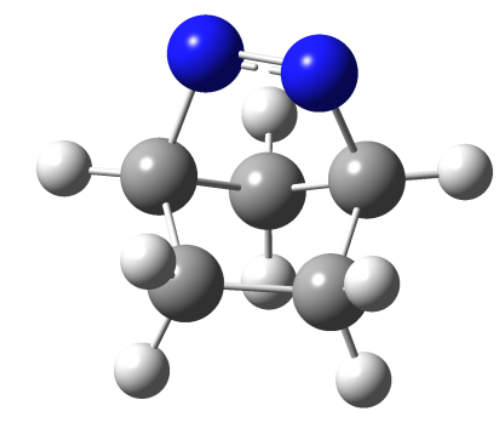

\begin{tabular}{|c|c|c|c|c|}
\hline Atom & No & $\begin{array}{l}\text { Alpha } \\
\text { Charge }\end{array}$ & $\begin{array}{l}\text { Beta } \\
\text { Charge }\end{array}$ & $\begin{array}{l}\text { Spin } \\
\text { Density }\end{array}$ \\
\hline $\mathrm{C}$ & 1 & -0.24953 & -0.23529 & 0.01424 \\
\hline $\mathrm{C}$ & 2 & -0.08381 & -0.0277 & 0.05611 \\
\hline $\mathrm{C}$ & 3 & -0.08951 & -0.01657 & 0.07294 \\
\hline $\mathrm{C}$ & 4 & -0.24755 & -0.21631 & 0.03124 \\
\hline $\mathrm{H}$ & 5 & 0.12655 & 0.12788 & 0.00133 \\
\hline $\mathrm{H}$ & 6 & 0.12218 & 0.12238 & 0.0002 \\
\hline $\mathrm{H}$ & 7 & 0.12849 & 0.12817 & -0.00032 \\
\hline $\mathrm{H}$ & 8 & 0.11008 & 0.12915 & 0.01907 \\
\hline $\mathrm{C}$ & 9 & -0.2642 & -0.21159 & 0.05261 \\
\hline $\mathrm{H}$ & 10 & 0.12986 & 0.12798 & -0.00188 \\
\hline $\mathrm{H}$ & 11 & 0.11965 & 0.11936 & -0.00029 \\
\hline $\mathrm{H}$ & 12 & 0.13185 & 0.12811 & -0.00374 \\
\hline $\mathrm{H}$ & 13 & 0.12836 & 0.13054 & 0.00218 \\
\hline $\mathrm{N}$ & 14 & -0.52773 & 0.31451 & 0.84224 \\
\hline $\mathrm{N}$ & 15 & -0.5347 & 0.37938 & 0.91408 \\
\hline
\end{tabular}


Figure S29. TS and IRC from $T_{1}$ of $\mathbf{A Z}$ to $T-1$

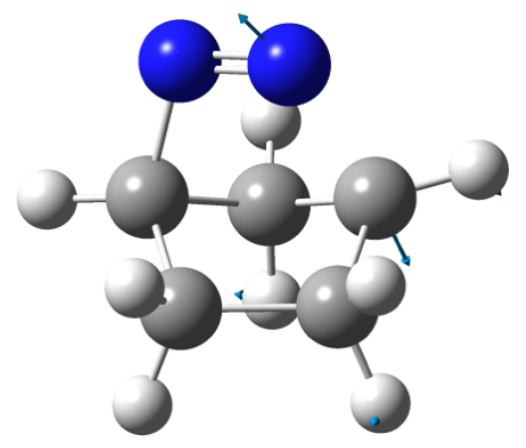

DFT/UB3LYP 6-31G(d)

$\mathrm{E}=-304.68731401$ a.u.

Zero-point correction $=0.125062$ a.u.

\# Imaginary frequency $=1$

Standard orientation:

\begin{tabular}{|c|c|c|c|c|}
\hline \multirow{2}{*}{$\begin{array}{l}\text { Center } \\
\text { Number }\end{array}$} & Atomic & \multirow{2}{*}{$\begin{array}{l}\text { Atomic } \\
\text { Type }\end{array}$} & \multicolumn{2}{|c|}{ Coordinates (Angstroms } \\
\hline & Numbe & & $\mathrm{X}$ & $\mathrm{Z}$ \\
\hline 1 & 6 & 0.871068 & 1.104095 & -0.383940 \\
\hline 2 & 6 & -0.484392 & 0.975254 & 0.349724 \\
\hline 3 & 6 & 0.350824 & -1.136830 & 0.338255 \\
\hline 4 & 6 & 1.425053 & -0.359409 & -0.396488 \\
\hline 5 & 1 & 0.739469 & 1.504747 & -1.392248 \\
\hline 6 & 1 & 1.532184 & 1.783229 & 0.165656 \\
\hline 7 & 1 & 1.591122 & -0.743784 & -1.405432 \\
\hline 8 & 1 & 2.375398 & -0.435375 & 0.154561 \\
\hline 9 & 6 & -0.209004 & -0.178053 & 1.367750 \\
\hline 10 & 1 & -1.119988 & -0.524829 & 1.860300 \\
\hline 11 & 1 & 0.537690 & 0.097871 & 2.123648 \\
\hline 12 & 1 & -0.914744 & 1.894856 & 0.745639 \\
\hline 13 & 1 & 0.417199 & -2.209439 & 0.490441 \\
\hline 14 & 7 & -0.929110 & -0.757994 & -1.014180 \\
\hline 15 & 7 & -1.482265 & 0.215476 & -0.470730 \\
\hline
\end{tabular}




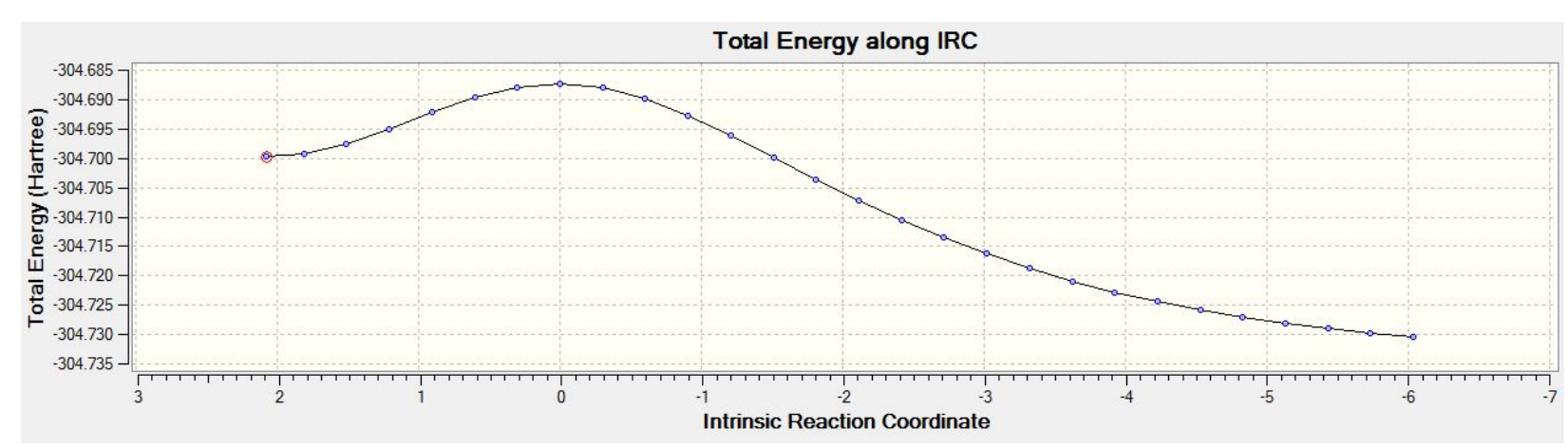

Figure S30. Optimization of T-1.

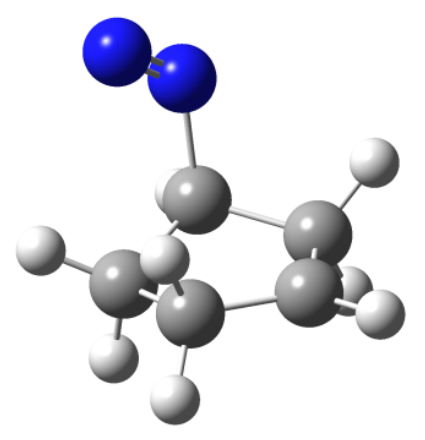

DFT/UB3LYP 6-31G(d)

$\mathrm{E}=-304.73316532$ a.u.

Zero-point correction $=0.122888$ a.u.

$<\mathrm{S} * * 2>=2.0088$

Standard orientation:

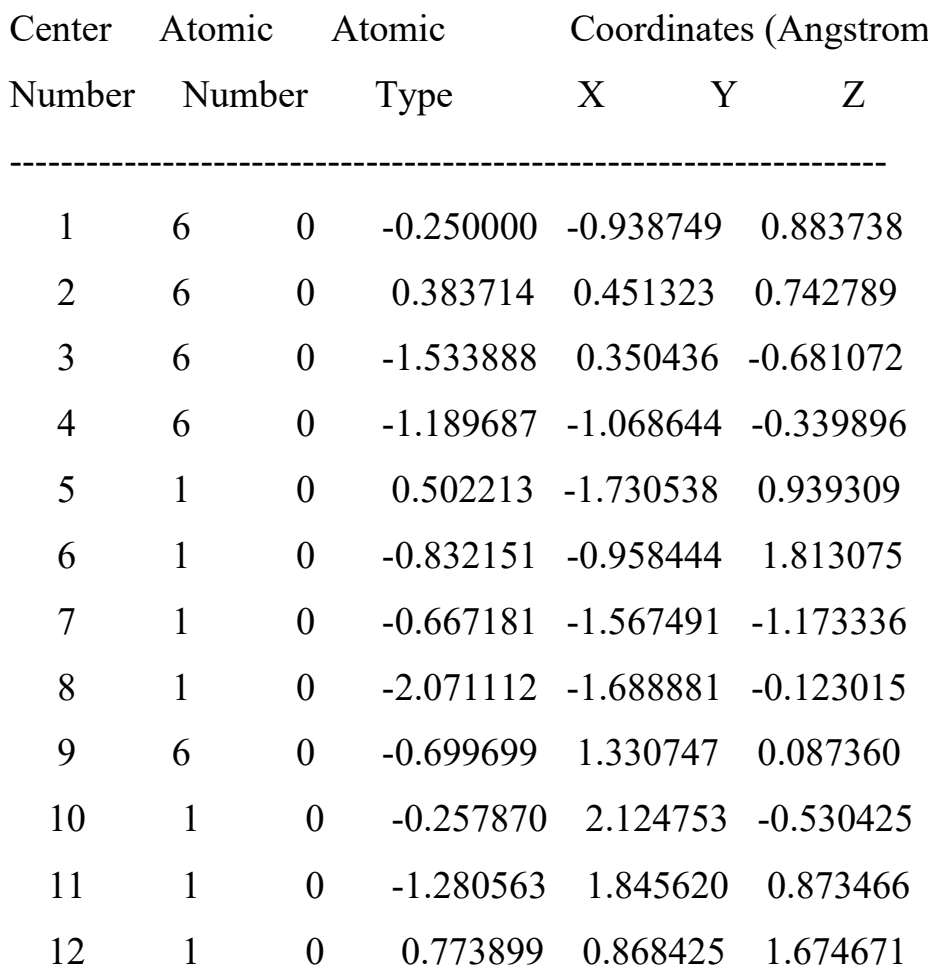




$\begin{array}{rrrrrr}13 & 1 & 0 & -2.275102 & 0.638526 & -1.418476 \\ 14 & 7 & 0 & 2.071520 & -0.502364 & -0.692431 \\ 15 & 7 & 0 & 1.620655 & 0.461986 & -0.195110\end{array}$

Table S4. Spin density of T-1.

\begin{tabular}{|c|c|c|c|c|}
\hline Atom & No & $\begin{array}{l}\text { Alpha } \\
\text { Charge }\end{array}$ & $\begin{array}{l}\text { Beta } \\
\text { Charge }\end{array}$ & $\begin{array}{l}\text { Spin } \\
\text { Density }\end{array}$ \\
\hline $\mathrm{C}$ & 1 & -0.24708 & -0.23386 & 0.01322 \\
\hline $\mathrm{C}$ & 2 & -0.13005 & 0.01986 & 0.14991 \\
\hline $\mathrm{C}$ & 3 & -0.54232 & 0.41396 & 0.95628 \\
\hline $\mathrm{C}$ & 4 & -0.2389 & -0.28501 & -0.04611 \\
\hline $\mathrm{H}$ & 5 & 0.12585 & 0.12896 & 0.00311 \\
\hline $\mathrm{H}$ & 6 & 0.12152 & 0.12322 & 0.0017 \\
\hline $\mathrm{H}$ & 7 & 0.10068 & 0.14946 & 0.04878 \\
\hline $\mathrm{H}$ & 8 & 0.10761 & 0.13921 & 0.0316 \\
\hline $\mathrm{C}$ & 9 & -0.23854 & -0.29221 & -0.05367 \\
\hline $\mathrm{H}$ & 10 & 0.11269 & 0.14573 & 0.03304 \\
\hline $\mathrm{H}$ & 11 & 0.09733 & 0.15157 & 0.05424 \\
\hline $\mathrm{H}$ & 12 & 0.13323 & 0.12752 & -0.00571 \\
\hline $\mathrm{H}$ & 13 & 0.11924 & 0.09136 & -0.02788 \\
\hline $\mathrm{N}$ & 14 & -0.36297 & 0.31916 & 0.68213 \\
\hline $\mathrm{N}$ & 15 & -0.1583 & 0.00107 & 0.15937 \\
\hline
\end{tabular}


Figure S31. TS and IRC from T-1 to T-DR.

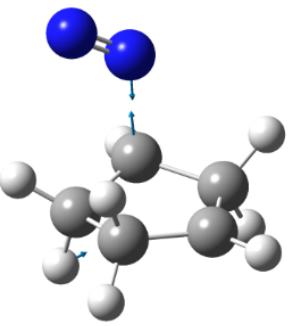

DFT/UB3LYP 6-31G(d)

$\mathrm{E}=-304.73107007$ a.u.

Zero-point correction $=0.120817$ a.u.

\# Imaginary frequency $=1$

Standard orientation:

\begin{tabular}{cccccc} 
Center & Atomic & \multicolumn{2}{c}{ Atomic } & \multicolumn{2}{c}{ Coordinates (Angstroms) } \\
Number & Number & Type & X & Y & $Z$ \\
- & & & & & \\
1 & 6 & 0 & -0.250351 & -0.974106 & 0.835094 \\
2 & 6 & 0 & 0.273039 & 0.448779 & 0.785314 \\
3 & 6 & 0 & -1.604350 & 0.331340 & -0.662638 \\
4 & 6 & 0 & -1.195804 & -1.085223 & -0.387944 \\
5 & 1 & 0 & 0.559353 & -1.711761 & 0.826552 \\
6 & 1 & 0 & -0.812079 & -1.113035 & 1.769266 \\
7 & 1 & 0 & -0.659045 & -1.522536 & -1.247393 \\
8 & 1 & 0 & -2.047789 & -1.752914 & -0.197130 \\
9 & 6 & 0 & -0.786673 & 1.314226 & 0.120829 \\
10 & 1 & 0 & -0.334545 & 2.107828 & -0.493714 \\
11 & 1 & 0 & -1.385490 & 1.840283 & 0.888209 \\
12 & 1 & 0 & 0.743563 & 0.843894 & 1.685491 \\
13 & 1 & 0 & -2.359457 & 0.616925 & -1.386900 \\
14 & 7 & 0 & 2.276612 & -0.442671 & -0.545921 \\
15 & 7 & 0 & 1.677720 & 0.511417 & -0.309553
\end{tabular}




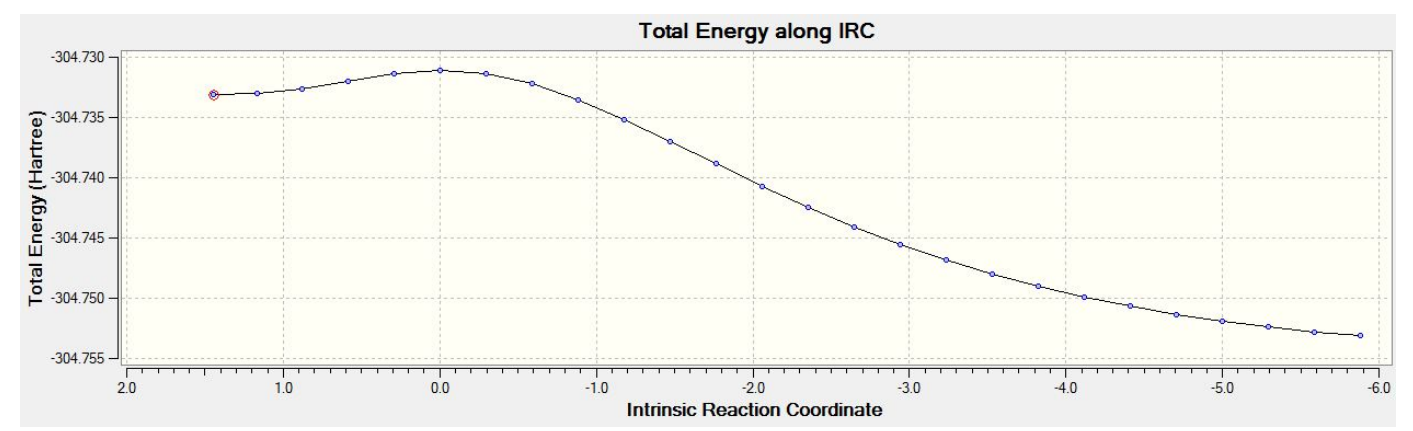

Figure S32. Optimization of T-DR.

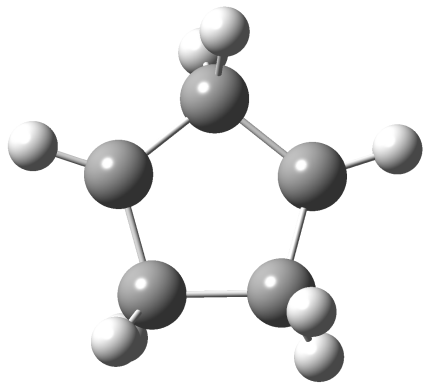

DFT/UB3LYP 6-31G(d)

$E=-195.22990749$ a.u.

Zero-point correction $=0.111089$ a.u.

$<\mathrm{S} * * 2>=2.0088$

Standard orientation:

\begin{tabular}{|c|c|c|c|c|c|}
\hline Center & Atomic & & omic & Coordinate & es (Angstro \\
\hline Number & Number & & Type & $X$ & $\mathrm{Z}$ \\
\hline 1 & 6 & 0 & -1.024807 & -0.772527 & 0.089782 \\
\hline 2 & 6 & 0 & 0.409993 & -1.173985 & -0.078394 \\
\hline 3 & 6 & 0 & 0.409825 & 1.174097 & 0.077871 \\
\hline 4 & 6 & 0 & -1.024988 & 0.772354 & -0.089655 \\
\hline 5 & 1 & 0 & -1.695583 & -1.277456 & -0.619841 \\
\hline 6 & 1 & 0 & -1.399889 & -1.041589 & 1.093574 \\
\hline 7 & 1 & 0 & -1.400568 & 1.041432 & -1.093253 \\
\hline 8 & 1 & 0 & -1.695558 & 1.277109 & 0.620307 \\
\hline 9 & 6 & 0 & 1.338626 & 0.000081 & 0.000189 \\
\hline 10 & 1 & 0 & 2.024772 & 0.053516 & -0.870685 \\
\hline 11 & 1 & 0 & 2.023713 & -0.053310 & 0.871966 \\
\hline 12 & 1 & 0 & 0.745769 & -2.205332 & -0.117743 \\
\hline 13 & 1 & 0 & 0.745448 & 2.205508 & 0.116915 \\
\hline
\end{tabular}


Figure S33. TD-DFT Calculation of T-DR.
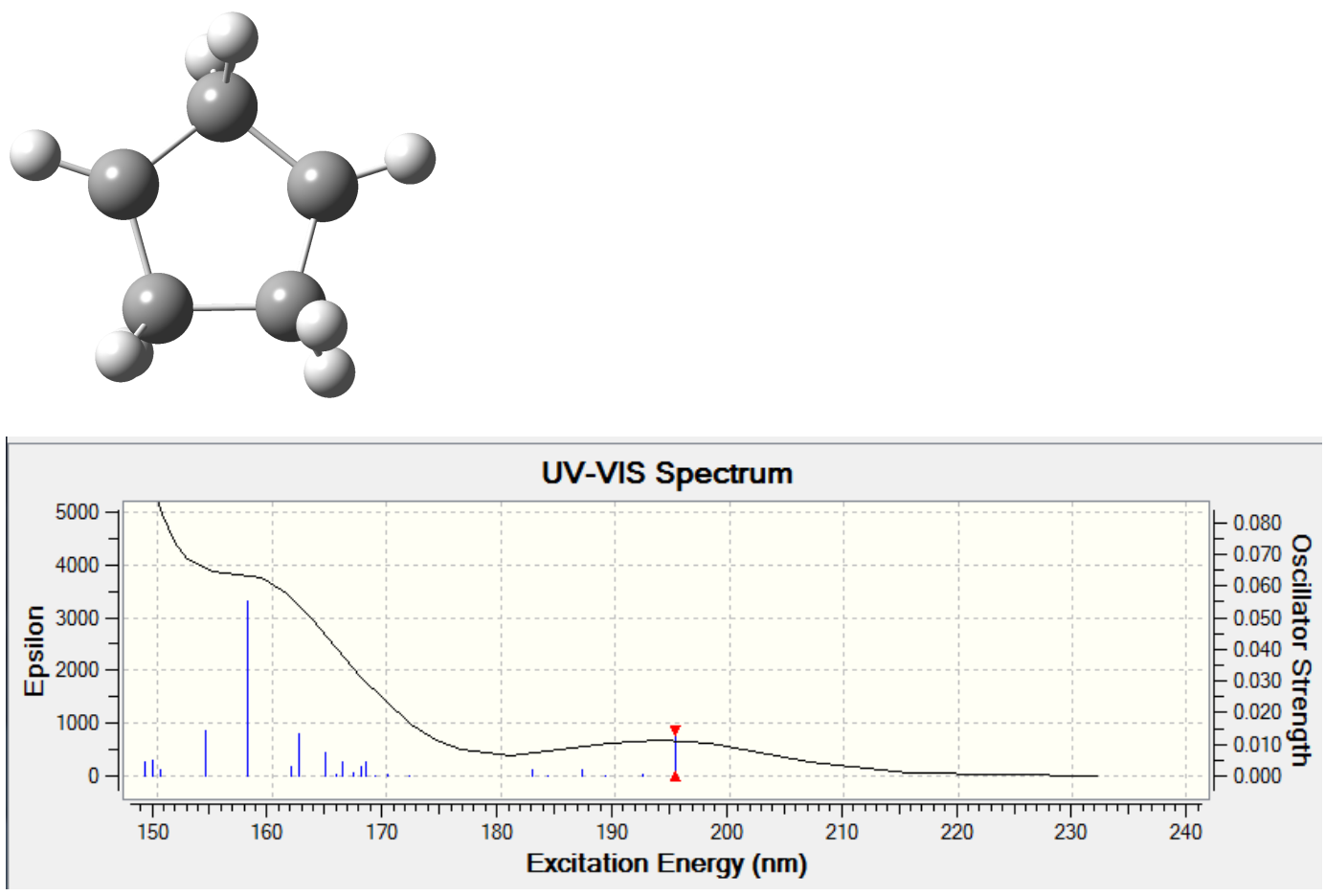

Table S5. Spin density of T-DR.

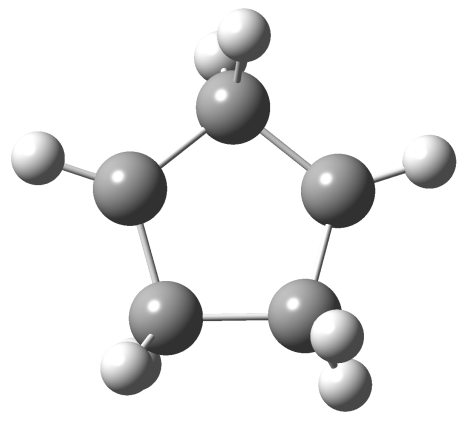

\begin{tabular}{lllll}
\hline Atom & No & $\begin{array}{l}\text { Alpha } \\
\text { Charge }\end{array}$ & Beta Charge & $\begin{array}{l}\text { Spin } \\
\text { Density }\end{array}$ \\
\hline $\mathrm{C}$ & 1 & -0.23652 & -0.27955 & -0.04303 \\
$\mathrm{C}$ & 2 & -0.5396 & 0.41608 & 0.95568 \\
$\mathrm{C}$ & 3 & -0.53961 & 0.41608 & 0.95569 \\
$\mathrm{C}$ & 4 & -0.23652 & -0.27954 & -0.04302 \\
$\mathrm{H}$ & 5 & 0.10698 & 0.13474 & 0.02776 \\
$\mathrm{H}$ & 6 & 0.09233 & 0.14553 & 0.0532 \\
$\mathrm{H}$ & 7 & 0.09234 & 0.14552 & 0.05318 \\
\hline
\end{tabular}




\begin{tabular}{lcccc}
\hline $\mathrm{H}$ & 8 & 0.10696 & 0.13475 & 0.02779 \\
$\mathrm{C}$ & 9 & -0.2437 & -0.33455 & -0.09085 \\
$\mathrm{H}$ & 10 & 0.08266 & 0.16267 & 0.08001 \\
$\mathrm{H}$ & 11 & 0.08258 & 0.16273 & 0.08015 \\
$\mathrm{H}$ & 12 & 0.11605 & 0.08777 & -0.02828 \\
$\mathrm{H}$ & 13 & 0.11605 & 0.08777 & -0.02828 \\
\hline
\end{tabular}

Figure S34. Optimization of S-DR.

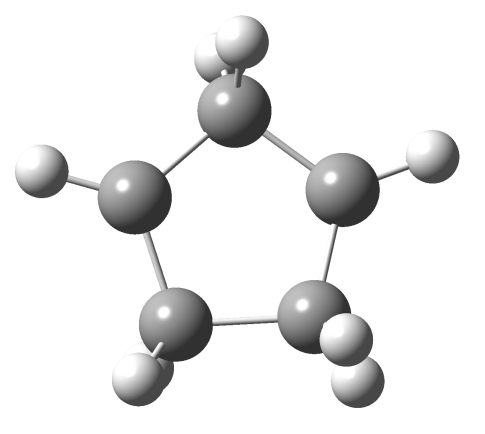

DFT/B3LYP 6-31G(d)

$\mathrm{E}=-195.22887915$ a.u.

Zero-point correction $=0.111193$ a.u.

$<\mathrm{S} * * 2>=1.0068$

Standard orientation:

\begin{tabular}{|c|c|c|c|c|}
\hline \multirow{2}{*}{$\begin{array}{l}\text { Center } \\
\text { Number }\end{array}$} & Atomic & \multirow{2}{*}{$\begin{array}{l}\text { Atomic } \\
\text { Type }\end{array}$} & \multicolumn{2}{|c|}{ Coordinates (Angstrom } \\
\hline & Numbe & & $X$ & Z \\
\hline 1 & 6 & -1.024841 & -0.772091 & 0.090696 \\
\hline 2 & 6 & 0.408574 & -1.174664 & -0.078850 \\
\hline 3 & 6 & 0.408406 & 1.174777 & 0.078289 \\
\hline 4 & 6 & -1.025023 & 0.771918 & -0.090561 \\
\hline 5 & 1 & -1.696980 & -1.278452 & -0.616749 \\
\hline 6 & 1 & -1.399191 & -1.038464 & 1.095661 \\
\hline 7 & 1 & -1.399913 & 1.038309 & -1.095315 \\
\hline 8 & 1 & -1.696933 & 1.278101 & 0.617252 \\
\hline 9 & 6 & 1.343292 & 0.000084 & 0.000194 \\
\hline 10 & 1 & 2.023503 & 0.053730 & -0.872452 \\
\hline
\end{tabular}




$\begin{array}{llllll}11 & 1 & 0 & 2.022343 & -0.053539 & 0.873839 \\ 12 & 1 & 0 & 0.742514 & -2.206615 & -0.119004 \\ 13 & 1 & 0 & 0.742203 & 2.206784 & 0.118167\end{array}$

Figure S35. TD-DFT Calculation of S-DR.

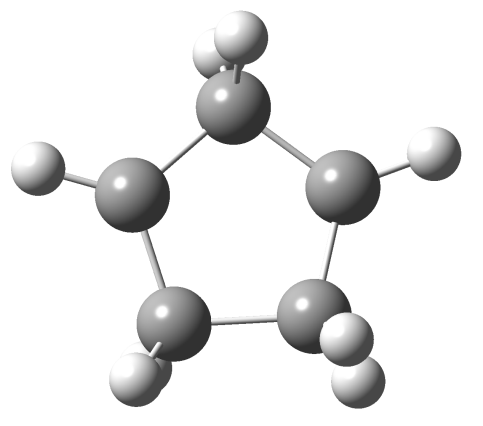

Excitation energies and oscillator strengths:

Excited State 1: $1.125-\mathrm{A} \quad 2.8894 \mathrm{eV} \quad 429.10 \mathrm{~nm} \mathrm{f}=0.0001<\mathrm{S} * * 2>=0.066$

$$
\begin{array}{cc}
19 \mathrm{~A}->20 \mathrm{~A} & -0.70493 \\
19 \mathrm{~B}->20 \mathrm{~B} & 0.70821
\end{array}
$$

Excited State $2: 1.115-\mathrm{A} \quad 3.0210 \mathrm{eV} \quad 410.41 \mathrm{~nm} \mathrm{f}=0.0032<\mathrm{S} * * 2>=0.061$

$$
\begin{array}{ll}
19 \mathrm{~A}->20 \mathrm{~A} & 0.70859 \\
19 \mathrm{~B}->20 \mathrm{~B} & 0.70531
\end{array}
$$

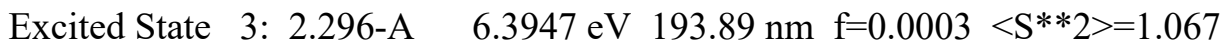

$$
\begin{array}{ll}
19 \mathrm{~A}->21 \mathrm{~A} & -0.68548 \\
19 \mathrm{~B}->21 \mathrm{~B} & 0.68900
\end{array}
$$

Excited State $4: 2.243-\mathrm{A} \quad 6.4467 \mathrm{eV} 192.32 \mathrm{~nm} \mathrm{f}=0.0147<\mathrm{S} * * 2>=1.008$

$$
\begin{array}{ll}
19 \mathrm{~A}->21 \mathrm{~A} & 0.68727 \\
19 \mathrm{~B}->21 \mathrm{~B} & 0.68377
\end{array}
$$

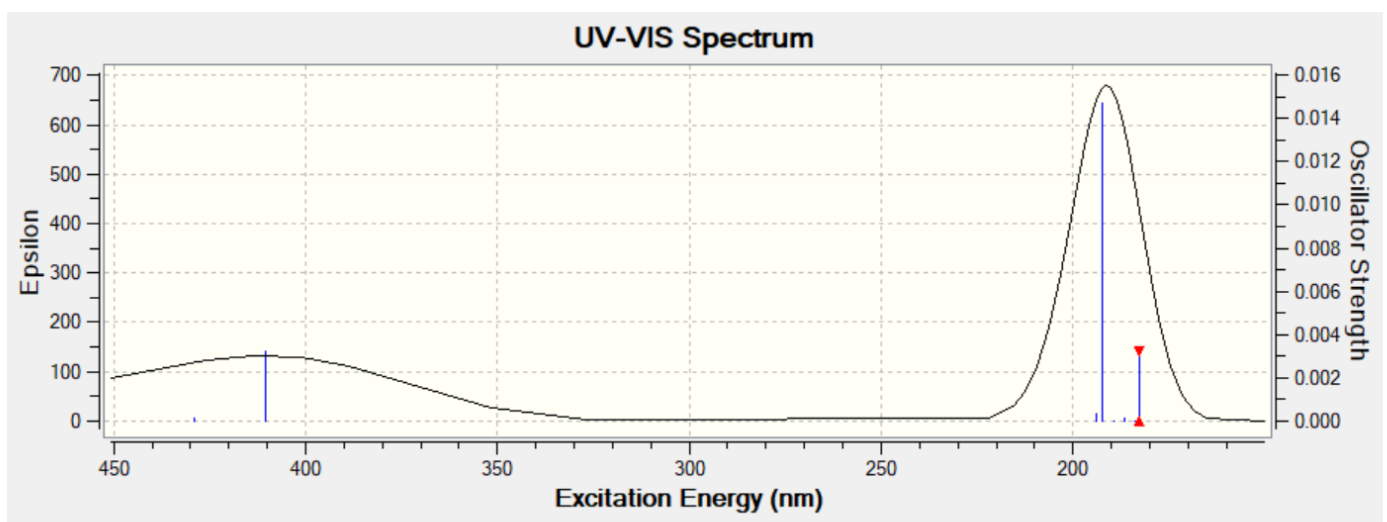


Figure S36. Optimization of CP.

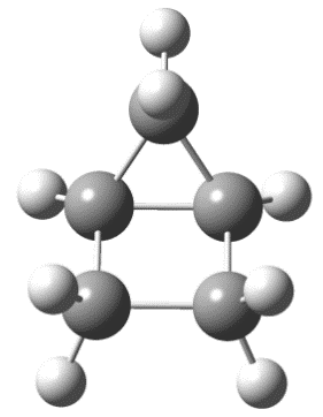

DFT/B3LYP 6-31G(d)

$\mathrm{E}=-195.28023453$ a.u.

Zero-point correction $=0.116865$ a.u.

Standard orientation:

\begin{tabular}{cccccc} 
Center & \multicolumn{2}{c}{ Atomic } & \multicolumn{2}{c}{ Atomic } & \multicolumn{2}{c}{ Coordinates (Angstroms) } \\
Number & Number & Type & X & Y & $Z$ \\
\hline 1 & 6 & 0 & 1.016629 & 0.783096 & 0.137087 \\
2 & 6 & 0 & -0.410352 & 0.766112 & -0.425820 \\
3 & 6 & 0 & -0.410118 & -0.766232 & -0.425709 \\
4 & 6 & 0 & 1.016780 & -0.782989 & 0.137108 \\
5 & 1 & 0 & 1.750749 & 1.194001 & -0.562290 \\
6 & 1 & 0 & 1.143556 & 1.280650 & 1.106764 \\
7 & 1 & 0 & 1.750866 & -1.193555 & -0.562498 \\
8 & 1 & 0 & 1.143981 & -1.280548 & 1.106741 \\
9 & 6 & 0 & -1.323682 & -0.000031 & 0.491319 \\
10 & 1 & 0 & -2.374443 & -0.000230 & 0.211015 \\
11 & 1 & 0 & -1.152191 & 0.000214 & 1.568906 \\
12 & 1 & 0 & -0.799074 & 1.448800 & -1.176428 \\
13 & 1 & 0 & -0.798980 & -1.449070 & -1.176124
\end{tabular}


Figure S37. Optimization of 3.

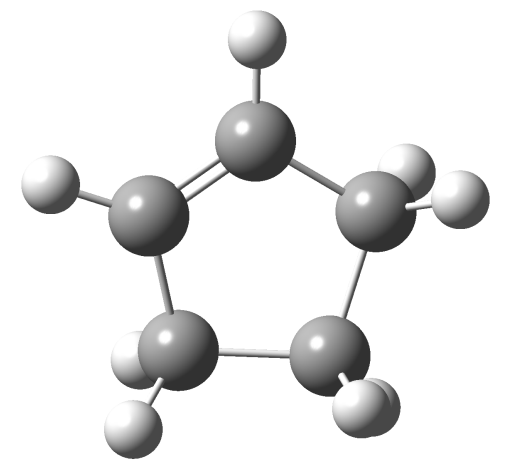

DFT/B3LYP 6-31G(d)

$E=-195.32714235$ a.u.

Zero-point correction $=0.117303$ a.u.

Standard orientation:

\begin{tabular}{crrrrr} 
Center & Atomic & \multicolumn{2}{c}{ Atomic } & \multicolumn{2}{c}{ Coordinates (Angstroms) } \\
Number & Number & Type & X & Y & $Z$ \\
\hline 1 & 6 & 0 & -0.256581 & -1.207826 & 0.000000 \\
2 & 6 & 0 & 0.066251 & 1.075000 & -0.667675 \\
3 & 6 & 0 & 0.066251 & -0.326241 & -1.237130 \\
4 & 1 & 0 & 0.293214 & -2.154206 & 0.000000 \\
5 & 1 & 0 & -1.324406 & -1.453983 & 0.000000 \\
6 & 1 & 0 & 1.050346 & -0.568189 & -1.667508 \\
7 & 1 & 0 & -0.662479 & -0.460139 & -2.046954 \\
8 & 6 & 0 & 0.066251 & 1.075000 & 0.667675 \\
9 & 1 & 0 & 0.102460 & 1.963349 & 1.293008 \\
10 & 1 & 0 & 0.102460 & 1.963349 & -1.293008 \\
11 & 6 & 0 & 0.066251 & -0.326241 & 1.237130 \\
12 & 1 & 0 & -0.662479 & -0.460139 & 2.046954 \\
13 & 1 & 0 & 1.050346 & -0.568189 & 1.667508 \\
& & & & &
\end{tabular}


Figure S38. Optimization of $S_{1}$ of $\mathbf{A Z}$.

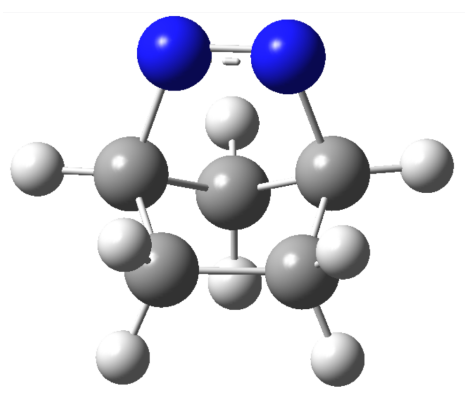

TD-DFT/B3LYP 6-31G(d)

$\mathrm{E}=-304.661394011$ a.u.

Zero-point correction $=0.126314$ a.u.

Standard orientation:

\begin{tabular}{|c|c|c|c|c|}
\hline Center & Atomic & Atomic & Coordinate & s (Angstroms \\
\hline Number & Number & Type & $\mathrm{X}$ & Z \\
\hline 1 & 6 & 0.217472 & -1.115694 & 0.240122 \\
\hline 2 & 1 & 0.453733 & -2.146106 & 0.499170 \\
\hline 3 & 6 & 0.333676 & -0.014618 & 1.336770 \\
\hline 4 & 1 & -0.434525 & -0.071344 & 2.116418 \\
\hline 5 & 1 & 1.328798 & 0.021226 & 1.784265 \\
\hline 6 & 6 & -1.177980 & -0.841974 & -0.366841 \\
\hline 7 & 1 & -1.245921 & -1.220248 & -1.389596 \\
\hline 8 & 1 & -1.949976 & -1.341821 & 0.232058 \\
\hline 9 & 6 & 0.079152 & 1.112093 & 0.306310 \\
\hline 10 & 1 & 0.203413 & 2.151514 & 0.607326 \\
\hline 11 & 6 & -1.289803 & 0.711014 & -0.283338 \\
\hline 12 & 1 & -1.464225 & 1.184235 & -1.251776 \\
\hline 13 & 1 & -2.087863 & 1.026649 & 0.404593 \\
\hline 14 & 7 & 1.246332 & -0.535166 & -0.692331 \\
\hline 15 & 7 & 1.071019 & 0.719590 & -0.793468 \\
\hline
\end{tabular}


Figure S39. TS from $S_{1}$ of $\mathbf{A Z}$ to $\mathrm{S}-\mathbf{1}$.

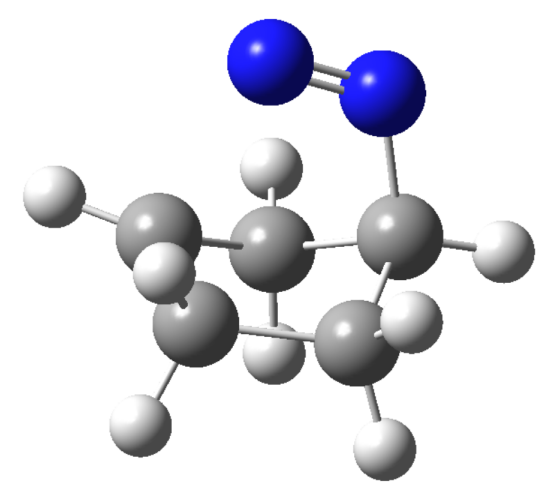

TD-DFT/B3LYP 6-31G(d)

$\mathrm{E}=-304.650288275$ a.u.

Zero-point correction $=0.124142$ a.u.

Standard orientation:

\begin{tabular}{ccccccc} 
Center & \multicolumn{2}{c}{ Atomic } & \multicolumn{2}{l}{ Atomic } & \multicolumn{2}{c}{ Coordinates (Angstroms) } \\
Number & Number & Type & X & $Y$ & $Z$ \\
\hline 1 & 6 & 0 & -0.565093 & -1.156064 & 0.255443 \\
2 & 1 & 0 & -0.648409 & -2.235996 & 0.249550 \\
3 & 6 & 0 & -0.007549 & -0.349356 & 1.367715 \\
4 & 1 & 0 & -0.784677 & -0.012938 & 2.070588 \\
5 & 1 & 0 & 0.799856 & -0.830605 & 1.925460 \\
6 & 6 & 0 & -1.349748 & -0.252110 & -0.650292 \\
7 & 1 & 0 & -1.227889 & -0.532075 & -1.702971 \\
8 & 1 & 0 & -2.429478 & -0.324387 & -0.426634 \\
9 & 6 & 0 & 0.475249 & 0.883612 & 0.515980 \\
10 & 1 & 0 & 0.870726 & 1.731143 & 1.074968 \\
11 & 6 & 0 & -0.794237 & 1.168618 & -0.339644 \\
12 & 1 & 0 & -0.551968 & 1.735041 & -1.242048 \\
13 & 1 & 0 & -1.504409 & 1.757891 & 0.252981 \\
14 & 7 & 0 & 1.195005 & -0.701936 & -0.990080 \\
15 & 7 & 0 & 1.508497 & 0.265326 & -0.309507
\end{tabular}


Figure S40. Optimization of S-1.

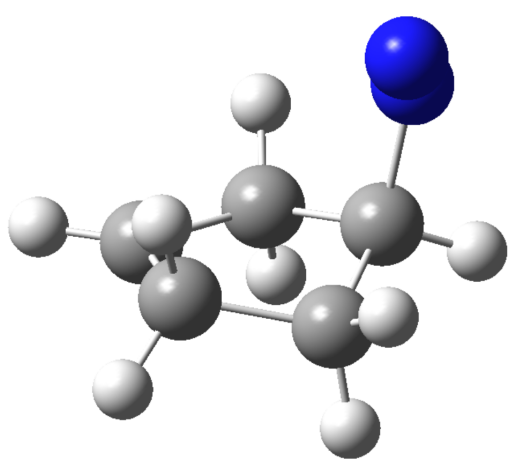

DFT/UB3LYP 6-31G(d)

$\mathrm{E}=-304.733413741$ a.u.

Zero-point correction $=0.122986$ a.u.

Standard orientation:

\begin{tabular}{cccccc} 
Center & \multicolumn{2}{c}{ Atomic } & \multicolumn{2}{c}{ Atomic } & \multicolumn{2}{c}{ Coordinates (Angstroms) } \\
Number & Number & Type & X & Y & $Z$ \\
\hline & & & & & \\
\hline & 6 & 0 & 1.548531 & 0.338619 & -0.666177 \\
2 & 1 & 0 & 2.324528 & 0.618854 & -1.370265 \\
3 & 6 & 0 & 0.704285 & 1.331010 & 0.077174 \\
4 & 1 & 0 & 1.276980 & 1.874134 & 0.849067 \\
5 & 1 & 0 & 0.252870 & 2.103265 & -0.562806 \\
6 & 6 & 0 & 1.171399 & -1.075280 & -0.345400 \\
7 & 1 & 0 & 0.624423 & -1.548295 & -1.179583 \\
8 & 1 & 0 & 2.038065 & -1.721419 & -0.147899 \\
9 & 6 & 0 & -0.379347 & 0.454572 & 0.740635 \\
10 & 1 & 0 & -0.767614 & 0.877352 & 1.670757 \\
11 & 6 & 0 & 0.246631 & -0.937575 & 0.886449 \\
12 & 1 & 0 & -0.510238 & -1.724280 & 0.952732 \\
13 & 1 & 0 & 0.838924 & -0.955638 & 1.809299 \\
14 & 7 & 0 & -2.077063 & -0.493901 & -0.687599 \\
15 & 7 & 0 & -1.612499 & 0.466465 & -0.194886
\end{tabular}


Figure S41. TS and IRC from S-1 to S-DR.

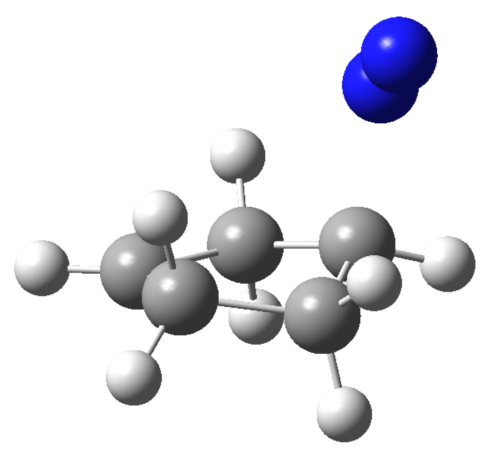

DFT/UB3LYP 6-31G(d)

$\mathrm{E}=-304.730894513$ a.u.

Zero-point correction $=0.120733$ a.u.

Standard orientation:

\begin{tabular}{cccccc} 
Center & \multicolumn{2}{c}{ Atomic } & \multicolumn{2}{c}{ Atomic } & \multicolumn{2}{c}{ Coordinates (Angstroms) } \\
Number & Number & Type & X & Y & $Z$ \\
\hline-1 & 6 & 0 & 1.605309 & 0.324896 & -0.661773 \\
2 & 1 & 0 & 2.385828 & 0.602684 & -1.361899 \\
3 & 6 & 0 & 0.782610 & 1.319346 & 0.105317 \\
4 & 1 & 0 & 1.380801 & 1.875277 & 0.850224 \\
5 & 1 & 0 & 0.310175 & 2.087627 & -0.527097 \\
6 & 6 & 0 & 1.184431 & -1.087365 & -0.390160 \\
7 & 1 & 0 & 0.629696 & -1.515538 & -1.243373 \\
8 & 1 & 0 & 2.031360 & -1.765054 & -0.213834 \\
9 & 6 & 0 & -0.263821 & 0.452410 & 0.793535 \\
10 & 1 & 0 & -0.730039 & 0.853050 & 1.693226 \\
11 & 6 & 0 & 0.255632 & -0.970213 & 0.843710 \\
12 & 1 & 0 & -0.556156 & -1.705928 & 0.847596 \\
13 & 1 & 0 & 0.829505 & -1.107896 & 1.770726 \\
14 & 7 & 0 & -2.264794 & -0.447876 & -0.557446 \\
15 & 7 & 0 & -1.687511 & 0.510923 & -0.293888
\end{tabular}




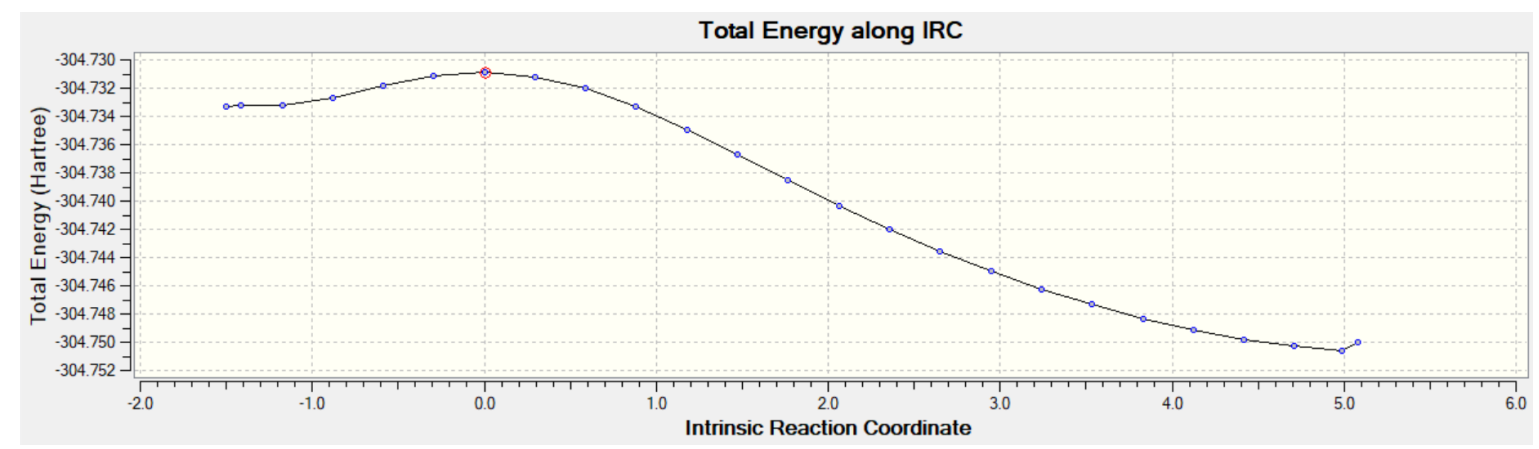

Figure S42. TS from S-DR to CP.

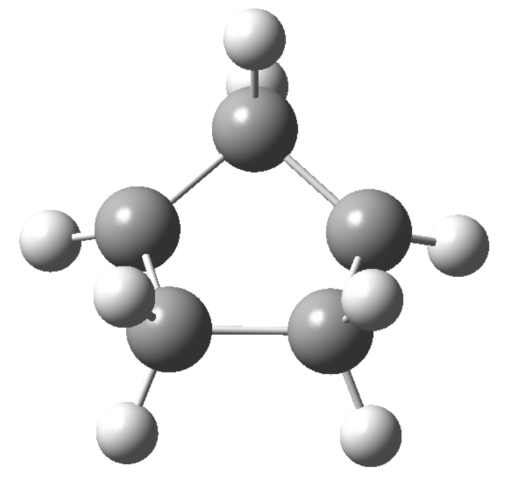

DFT/UB3LYP 6-31G(d)

$E=-195.224068335$ a.u.

Zero-point correction $=0.111200$ a.u.

Standard orientation:

\begin{tabular}{cccccc} 
Center & \multicolumn{2}{c}{ Atomic } & \multicolumn{2}{c}{ Atomic } & \multicolumn{2}{c}{ Coordinates (Angstrom } \\
Number & Number & Type & X & Y & Z \\
& & & & & \\
- & & & & & \\
1 & 6 & 0 & -1.334298 & -0.000000 & 0.239031 \\
2 & 1 & 0 & -1.568306 & -0.000000 & 1.323589 \\
3 & 1 & 0 & -2.295056 & -0.000000 & -0.289913 \\
4 & 6 & 0 & -0.421503 & 1.116647 & -0.188740 \\
5 & 1 & 0 & -0.744553 & 1.965322 & -0.783016 \\
6 & 6 & 0 & -0.421503 & -1.116647 & -0.188740 \\
7 & 1 & 0 & -0.744553 & -1.965322 & -0.783016 \\
8 & 6 & 0 & 1.022259 & -0.778614 & 0.063978 \\
9 & 1 & 0 & 1.680401 & -1.174812 & -0.718682 \\
10 & 1 & 0 & 1.394188 & -1.195315 & 1.016336 \\
11 & 6 & 0 & 1.022259 & 0.778614 & 0.063978 \\
12 & 1 & 0 & 1.394188 & 1.195315 & 1.016336
\end{tabular}


$\begin{array}{llllll}13 & 1 & 0 & 1.680401 & 1.174812 & -0.718682\end{array}$

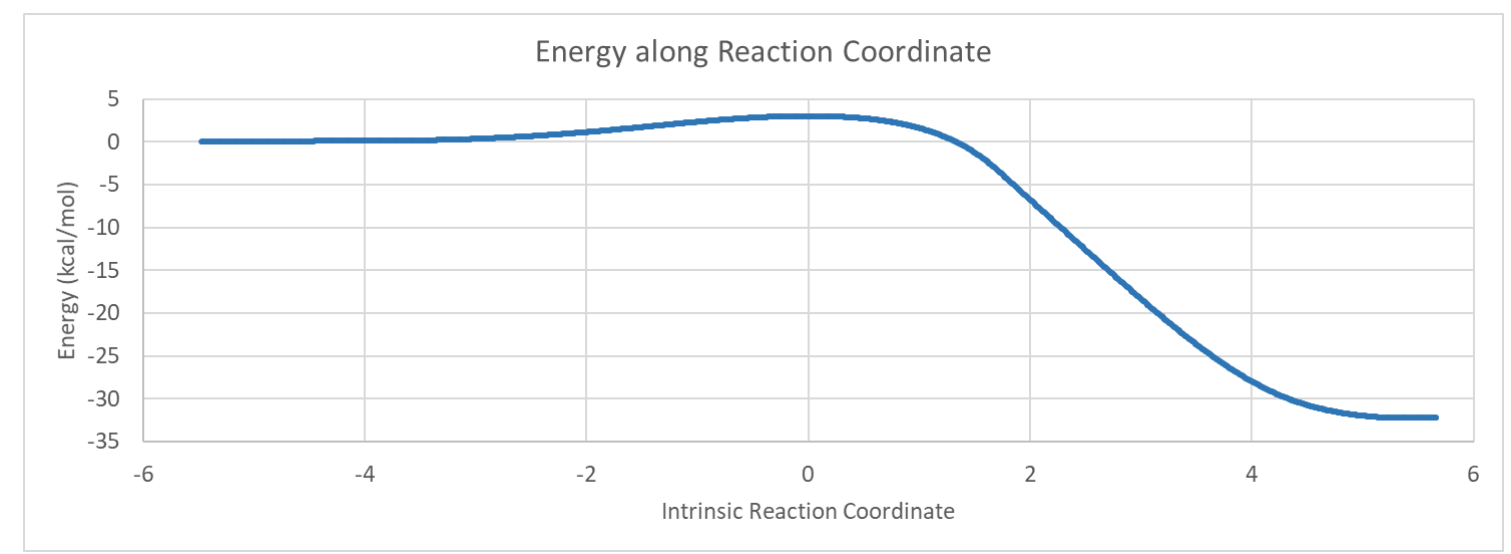

\section{Polyrate Output Files (poly.fu15):}

$\mathrm{S}_{1}$ of $\mathbf{A Z}$ reaction on the excited singlet surface:

Summary of forward rate constants $\left(\mathrm{s}^{-1}\right)$ :

\section{$\mathrm{T}(\mathrm{K}) \quad \mathrm{TST} \quad \mathrm{CVT} \quad \mathrm{CVT} / \mathrm{ZCT} \quad \mathrm{CVT} / \mathrm{SCT}$}

6.00 9.16E-194 3.98E-196 8.24E-12 1.46E-10

$8.00 \quad 1.35 \mathrm{E}-142 \quad 2.29 \mathrm{E}-144 \quad 8.24 \mathrm{E}-12 \quad 1.46 \mathrm{E}-10$

10.00 7.16E-112 2.73E-113 8.24E-12 1.46E-10

20.00 2.57E-50 4.98E-51 8.26E-12 1.46E-10

30.00 1.01E-29 3.36E-30 8.70E-12 1.51E-10

$40.00 \quad 2.16 \mathrm{E}-19$ 9.47E-20 $1.15 \mathrm{E}-11 \quad 1.80 \mathrm{E}-10$

50.00 3.57E-13 1.84E-13 3.83E-11 3.32E-10

75.00 7.55E-05 4.83E-05 1.50E-04 1.59E-04

77.36 2.44E-04 1.58E-04 4.62E-04 4.86E-04

$100.00 \quad 1.16 \mathrm{E}+00 \quad 8.29 \mathrm{E}-01 \quad 1.71 \mathrm{E}+00 \quad 1.74 \mathrm{E}+00$

$\begin{array}{lll}125.00 & 3.93 \mathrm{E}+02 \quad 2.97 \mathrm{E}+02 \quad 5.09 \mathrm{E}+02 \quad 5.15 \mathrm{E}+02\end{array}$

$\begin{array}{lllll}150.00 & 1.95 \mathrm{E}+04 & 1.53 \mathrm{E}+04 & 2.36 \mathrm{E}+04 & 2.37 \mathrm{E}+04\end{array}$

$\begin{array}{llll}175.00 & 3.22 \mathrm{E}+05 \quad 2.61 \mathrm{E}+05 \quad 3.72 \mathrm{E}+05 \quad 3.74 \mathrm{E}+05\end{array}$

$194.70 \quad 1.79 \mathrm{E}+06 \quad 1.47 \mathrm{E}+06 \quad 2.01 \mathrm{E}+06 \quad 2.01 \mathrm{E}+06$

$200.00 \quad 2.68 \mathrm{E}+06 \quad 2.21 \mathrm{E}+06 \quad 2.99 \mathrm{E}+06 \quad 3.00 \mathrm{E}+06$

$225.00 \quad 1.40 \mathrm{E}+07 \quad 1.17 \mathrm{E}+07 \quad 1.53 \mathrm{E}+07 \quad 1.53 \mathrm{E}+07$

$250.00 \quad 5.32 \mathrm{E}+07 \quad 4.49 \mathrm{E}+07 \quad 5.67 \mathrm{E}+07 \quad 5.68 \mathrm{E}+07$ 
$273.15 \quad 1.48 \mathrm{E}+08 \quad 1.26 \mathrm{E}+08 \quad 1.55 \mathrm{E}+08 \quad 1.55 \mathrm{E}+08$ $275.00 \quad 1.60 \mathrm{E}+08 \quad 1.36 \mathrm{E}+08 \quad 1.67 \mathrm{E}+08 \quad 1.67 \mathrm{E}+08$

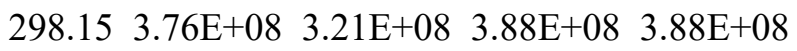
$300.00 \quad 4.01 \mathrm{E}+08 \quad 3.42 \mathrm{E}+08 \quad 4.13 \mathrm{E}+08 \quad 4.13 \mathrm{E}+08$ $325.00 \quad 8.77 \mathrm{E}+08 \quad 7.51 \mathrm{E}+08 \quad 8.90 \mathrm{E}+08 \quad 8.91 \mathrm{E}+08$

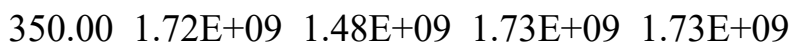

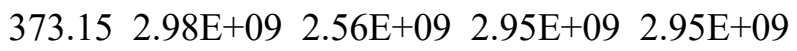

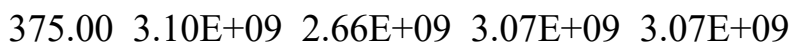
$\begin{array}{lllll}400.00 & 5.20 \mathrm{E}+09 & 4.47 \mathrm{E}+09 & 5.09 \mathrm{E}+09 & 5.09 \mathrm{E}+09\end{array}$

$\mathrm{T}_{1}$ of $\mathbf{A Z}$ to $\mathrm{T}-1$ :

Summary of forward rate constants $\left(\mathrm{s}^{-1}\right)$ :

$\mathrm{T}(\mathrm{K}) \quad \mathrm{TST} \quad \mathrm{CVT} \quad \mathrm{CVT} / \mathrm{ZCT} \quad \mathrm{CVT} / \mathrm{SCT}$

$6.00 \quad 2.09 \mathrm{E}-224 \quad 9.62 \mathrm{E}-225 \quad 4.69 \mathrm{E}+01 \quad 1.31 \mathrm{E}+02$ $8.00 \quad 1.40 \mathrm{E}-165 \quad 7.80 \mathrm{E}-166 \quad 4.69 \mathrm{E}+01 \quad 1.31 \mathrm{E}+02$ $\begin{array}{llll}10.00 & 2.90 \mathrm{E}-130 & 1.82 \mathrm{E}-130 & 4.69 \mathrm{E}+01 \quad 1.31 \mathrm{E}+02\end{array}$ $20.00 \quad 1.60 \mathrm{E}-59 \quad 1.26 \mathrm{E}-59 \quad 4.69 \mathrm{E}+01 \quad 1.31 \mathrm{E}+02$ $30.00 \quad 7.24 \mathrm{E}-36 \quad 6.19 \mathrm{E}-36 \quad 4.70 \mathrm{E}+01 \quad 1.31 \mathrm{E}+02$ $40.00 \quad 5.31 \mathrm{E}-24 \quad 4.72 \mathrm{E}-24 \quad 4.71 \mathrm{E}+01 \quad 1.32 \mathrm{E}+02$ $50.00 \quad 7.34 \mathrm{E}-17 \quad 6.68 \mathrm{E}-17 \quad 4.79 \mathrm{E}+01 \quad 1.33 \mathrm{E}+02$

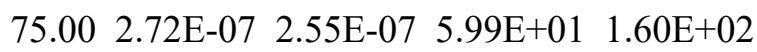

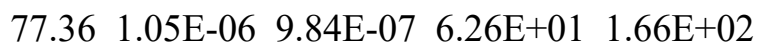
$100.00 \quad 1.81 \mathrm{E}-02 \quad 1.72 \mathrm{E}-02 \quad 1.29 \mathrm{E}+02 \quad 2.94 \mathrm{E}+02$ $125.00 \quad 1.49 \mathrm{E}+01 \quad 1.43 \mathrm{E}+01 \quad 7.97 \mathrm{E}+02 \quad 1.28 \mathrm{E}+03$ $\begin{array}{lllll}150.00 & 1.36 \mathrm{E}+03 \quad 1.32 \mathrm{E}+03 & 1.07 \mathrm{E}+04 & 1.32 \mathrm{E}+04\end{array}$ $\begin{array}{llll}175.00 & 3.52 \mathrm{E}+04 & 3.42 \mathrm{E}+04 & 1.21 \mathrm{E}+05 \quad 1.36 \mathrm{E}+05\end{array}$ $\begin{array}{llll}194.70 & 2.57 \mathrm{E}+05 \quad 2.50 \mathrm{E}+05 \quad 6.20 \mathrm{E}+05 \quad 6.71 \mathrm{E}+05\end{array}$ $200.00 \quad 4.11 \mathrm{E}+05 \quad 4.00 \mathrm{E}+05 \quad 9.23 \mathrm{E}+05 \quad 9.91 \mathrm{E}+05$ $225.00 \quad 2.83 \mathrm{E}+06 \quad 2.75 \mathrm{E}+06 \quad 4.91 \mathrm{E}+06 \quad 5.16 \mathrm{E}+06$ $250.00 \quad 1.34 \mathrm{E}+07 \quad 1.30 \mathrm{E}+07 \quad 1.97 \mathrm{E}+07 \quad 2.04 \mathrm{E}+07$ $273.15 \quad 4.40 \mathrm{E}+07 \quad 4.30 \mathrm{E}+07 \quad 5.85 \mathrm{E}+07 \quad 6.02 \mathrm{E}+07$ $\begin{array}{lllll}275.00 & 4.80 \mathrm{E}+07 & 4.69 \mathrm{E}+07 & 6.33 \mathrm{E}+07 & 6.52 \mathrm{E}+07\end{array}$ $298.15 \quad 1.31 \mathrm{E}+08 \quad 1.28 \mathrm{E}+08 \quad 1.60 \mathrm{E}+08 \quad 1.64 \mathrm{E}+08$ $300.00 \quad 1.41 \mathrm{E}+08 \quad 1.37 \mathrm{E}+08 \quad 1.71 \mathrm{E}+08 \quad 1.75 \mathrm{E}+08$

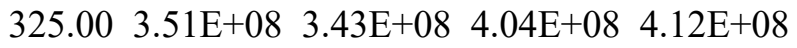


$350.00 \quad 7.71 \mathrm{E}+08 \quad 7.54 \mathrm{E}+08 \quad 8.52 \mathrm{E}+08 \quad 8.66 \mathrm{E}+08$ $373.15 \quad 1.46 \mathrm{E}+09 \quad 1.43 \mathrm{E}+09 \quad 1.57 \mathrm{E}+09 \quad 1.59 \mathrm{E}+09$

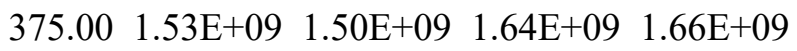
$\begin{array}{llll}400.00 & 2.80 \mathrm{E}+09 & 2.74 \mathrm{E}+09 & 2.93 \mathrm{E}+09 \\ 2.96 \mathrm{E}+09\end{array}$

\section{S-1 to S-DR:}

Summary of forward rate constants $\left(\mathrm{s}^{-1}\right)$ :

$$
\begin{array}{llllll}
\mathrm{T}(\mathrm{K}) & \mathrm{TST} & \mathrm{CVT} & \mathrm{CVT} / \mathrm{ZCT} & \mathrm{CVT} / \mathrm{SCT} \\
& & & & & \\
6.00 & 1.11 \mathrm{E}+05 & 7.59 \mathrm{E}+03 & 5.68 \mathrm{E}+11 & 5.71 \mathrm{E}+11 \\
8.00 & 4.92 \mathrm{E}+06 & 6.56 \mathrm{E}+05 & 5.68 \mathrm{E}+11 & 5.71 \mathrm{E}+11 \\
10.00 & 5.03 \mathrm{E}+07 & 1.00 \mathrm{E}+07 & 5.68 \mathrm{E}+11 & 5.71 \mathrm{E}+11 \\
20.00 & 6.78 \mathrm{E}+09 & 3.01 \mathrm{E}+09 & 5.73 \mathrm{E}+11 & 5.76 \mathrm{E}+11 \\
30.00 & 4.20 \mathrm{E}+10 & 2.43 \mathrm{E}+10 & 5.93 \mathrm{E}+11 & 5.96 \mathrm{E}+11 \\
40.00 & 1.15 \mathrm{E}+11 & 7.60 \mathrm{E}+10 & 6.36 \mathrm{E}+11 & 6.39 \mathrm{E}+11 \\
50.00 & 2.25 \mathrm{E}+11 & 1.60 \mathrm{E}+11 & 7.04 \mathrm{E}+11 & 7.07 \mathrm{E}+11 \\
75.00 & 6.28 \mathrm{E}+11 & 4.90 \mathrm{E}+11 & 9.76 \mathrm{E}+11 & 9.79 \mathrm{E}+11 \\
77.36 & 6.74 \mathrm{E}+11 & 5.29 \mathrm{E}+11 & 1.01 \mathrm{E}+12 & 1.01 \mathrm{E}+12 \\
100.00 & 1.17 \mathrm{E}+12 & 9.52 \mathrm{E}+11 & 1.37 \mathrm{E}+12 & 1.37 \mathrm{E}+12 \\
125.00 & 1.81 \mathrm{E}+12 & 1.51 \mathrm{E}+12 & 1.86 \mathrm{E}+12 & 1.87 \mathrm{E}+12 \\
150.00 & 2.54 \mathrm{E}+12 & 2.13 \mathrm{E}+12 & 2.42 \mathrm{E}+12 & 2.43 \mathrm{E}+12 \\
175.00 & 3.31 \mathrm{E}+12 & 2.21 \mathrm{E}+12 & 1.07 \mathrm{E}+12 & 1.07 \mathrm{E}+12 \\
194.70 & 3.95 \mathrm{E}+12 & 2.22 \mathrm{E}+12 & 1.14 \mathrm{E}+12 & 1.14 \mathrm{E}+12 \\
200.00 & 4.13 \mathrm{E}+12 & 2.23 \mathrm{E}+12 & 1.16 \mathrm{E}+12 & 1.16 \mathrm{E}+12 \\
225.00 & 4.97 \mathrm{E}+12 & 2.23 \mathrm{E}+12 & 1.16 \mathrm{E}+12 & 1.16 \mathrm{E}+12 \\
250.00 & 5.82 \mathrm{E}+12 & 2.22 \mathrm{E}+12 & 1.23 \mathrm{E}+12 & 1.23 \mathrm{E}+12 \\
273.15 & 6.61 \mathrm{E}+12 & 2.22 \mathrm{E}+12 & 1.28 \mathrm{E}+12 & 1.28 \mathrm{E}+12 \\
275.00 & 6.67 \mathrm{E}+12 & 2.22 \mathrm{E}+12 & 1.29 \mathrm{E}+12 & 1.29 \mathrm{E}+12 \\
298.15 & 7.46 \mathrm{E}+12 & 2.22 \mathrm{E}+12 & 1.33 \mathrm{E}+12 & 1.33 \mathrm{E}+12 \\
300.00 & 7.53 \mathrm{E}+12 & 2.22 \mathrm{E}+12 & 1.34 \mathrm{E}+12 & 1.34 \mathrm{E}+12 \\
325.00 & 8.37 \mathrm{E}+12 & 2.21 \mathrm{E}+12 & 1.38 \mathrm{E}+12 & 1.39 \mathrm{E}+12 \\
350.00 & 9.20 \mathrm{E}+12 & 2.21 \mathrm{E}+12 & 1.43 \mathrm{E}+12 & 1.43 \mathrm{E}+12 \\
373.15 & 9.96 \mathrm{E}+12 & 2.21 \mathrm{E}+12 & 1.46 \mathrm{E}+12 & 1.46 \mathrm{E}+12 \\
375.00 & 1.00 \mathrm{E}+13 & 2.21 \mathrm{E}+12 & 1.46 \mathrm{E}+12 & 1.47 \mathrm{E}+12 \\
400.00 & 1.08 \mathrm{E}+13 & 2.21 \mathrm{E}+12 & 1.50 \mathrm{E}+12 & 1.50 \mathrm{E}+12
\end{array}
$$




\section{T-1 to T-DR:}

Summary of forward rate constants $\left(\mathrm{s}^{-1}\right)$ :

\section{T(K) TST CVT CVT/ZCT $\quad$ CVT/SCT}

$6.00 \quad 1.92 \mathrm{E}+12 \quad 1.12 \mathrm{E}+11 \quad 9.87 \mathrm{E}+11 \quad 9.87 \mathrm{E}+11$

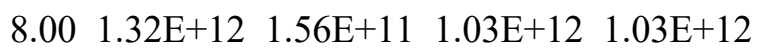

$10.00 \quad 1.11 \mathrm{E}+12 \quad 2.00 \mathrm{E}+11 \quad 1.07 \mathrm{E}+12 \quad 1.07 \mathrm{E}+12$

$20.00 \quad 9.91 \mathrm{E}+11 \quad 4.20 \mathrm{E}+11 \quad 1.29 \mathrm{E}+12 \quad 1.29 \mathrm{E}+12$

$30.00 \quad 1.13 \mathrm{E}+12 \quad 6.35 \mathrm{E}+11 \quad 1.49 \mathrm{E}+12 \quad 1.49 \mathrm{E}+12$

$40.00 \quad 1.32 \mathrm{E}+12 \quad 8.48 \mathrm{E}+11 \quad 1.70 \mathrm{E}+12 \quad 1.70 \mathrm{E}+12$

$50.00 \quad 1.53 \mathrm{E}+12 \quad 1.06 \mathrm{E}+12 \quad 1.91 \mathrm{E}+12 \quad 1.91 \mathrm{E}+12$

$\begin{array}{llll}75.00 & 2.10 \mathrm{E}+12 \quad 1.62 \mathrm{E}+12 \quad 2.47 \mathrm{E}+12 \quad 2.47 \mathrm{E}+12\end{array}$

$\begin{array}{llll}77.36 & 2.16 \mathrm{E}+12 \quad 1.67 \mathrm{E}+12 \quad 2.53 \mathrm{E}+12 \quad 2.53 \mathrm{E}+12\end{array}$

$100.00 \quad 2.73 \mathrm{E}+12 \quad 1.80 \mathrm{E}+12 \quad 1.66 \mathrm{E}+12 \quad 1.66 \mathrm{E}+12$

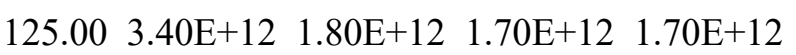

$\begin{array}{lll}150.00 & 4.10 \mathrm{E}+12 \quad 1.81 \mathrm{E}+12 \quad 1.73 \mathrm{E}+12 \quad 1.73 \mathrm{E}+12\end{array}$

$175.00 \quad 4.81 \mathrm{E}+12 \quad 1.81 \mathrm{E}+12 \quad 1.75 \mathrm{E}+12 \quad 1.75 \mathrm{E}+12$

$194.70 \quad 5.37 \mathrm{E}+12 \quad 1.82 \mathrm{E}+12 \quad 1.76 \mathrm{E}+12 \quad 1.76 \mathrm{E}+12$

$200.00 \quad 5.52 \mathrm{E}+12 \quad 1.82 \mathrm{E}+12 \quad 1.68 \mathrm{E}+12 \quad 1.68 \mathrm{E}+12$

$225.00 \quad 6.23 \mathrm{E}+12 \quad 1.81 \mathrm{E}+12 \quad 1.69 \mathrm{E}+12 \quad 1.69 \mathrm{E}+12$

$250.00 \quad 6.92 \mathrm{E}+12 \quad 1.80 \mathrm{E}+12 \quad 1.70 \mathrm{E}+12 \quad 1.70 \mathrm{E}+12$

$273.15 \quad 7.55 \mathrm{E}+12 \quad 1.79 \mathrm{E}+12 \quad 1.70 \mathrm{E}+12 \quad 1.70 \mathrm{E}+12$

$275.00 \quad 7.60 \mathrm{E}+12 \quad 1.79 \mathrm{E}+12 \quad 1.70 \mathrm{E}+12 \quad 1.70 \mathrm{E}+12$

$298.15 \quad 8.22 \mathrm{E}+12 \quad 1.79 \mathrm{E}+12 \quad 1.71 \mathrm{E}+12 \quad 1.71 \mathrm{E}+12$

$300.00 \quad 8.27 \mathrm{E}+12 \quad 1.79 \mathrm{E}+12 \quad 1.71 \mathrm{E}+12 \quad 1.71 \mathrm{E}+12$

$325.00 \quad 8.91 \mathrm{E}+12 \quad 1.79 \mathrm{E}+12 \quad 1.71 \mathrm{E}+12 \quad 1.71 \mathrm{E}+12$

$350.00 \quad 9.53 \mathrm{E}+12 \quad 1.78 \mathrm{E}+12 \quad 1.71 \mathrm{E}+12 \quad 1.71 \mathrm{E}+12$

$373.15 \quad 1.01 \mathrm{E}+13 \quad 1.78 \mathrm{E}+12 \quad 1.72 \mathrm{E}+12 \quad 1.72 \mathrm{E}+12$

$375.00 \quad 1.01 \mathrm{E}+13 \quad 1.78 \mathrm{E}+12 \quad 1.72 \mathrm{E}+12 \quad 1.72 \mathrm{E}+12$

$\begin{array}{lllll}400.00 & 1.07 \mathrm{E}+13 \quad 1.78 \mathrm{E}+12 & 1.72 \mathrm{E}+12 & 1.72 \mathrm{E}+12\end{array}$

\section{S-DR to CP:}

Summary of forward rate constants $\left(\mathrm{s}^{-1}\right)$ :

T(K) TST CVT CVT/ZCT CVT/SCT 


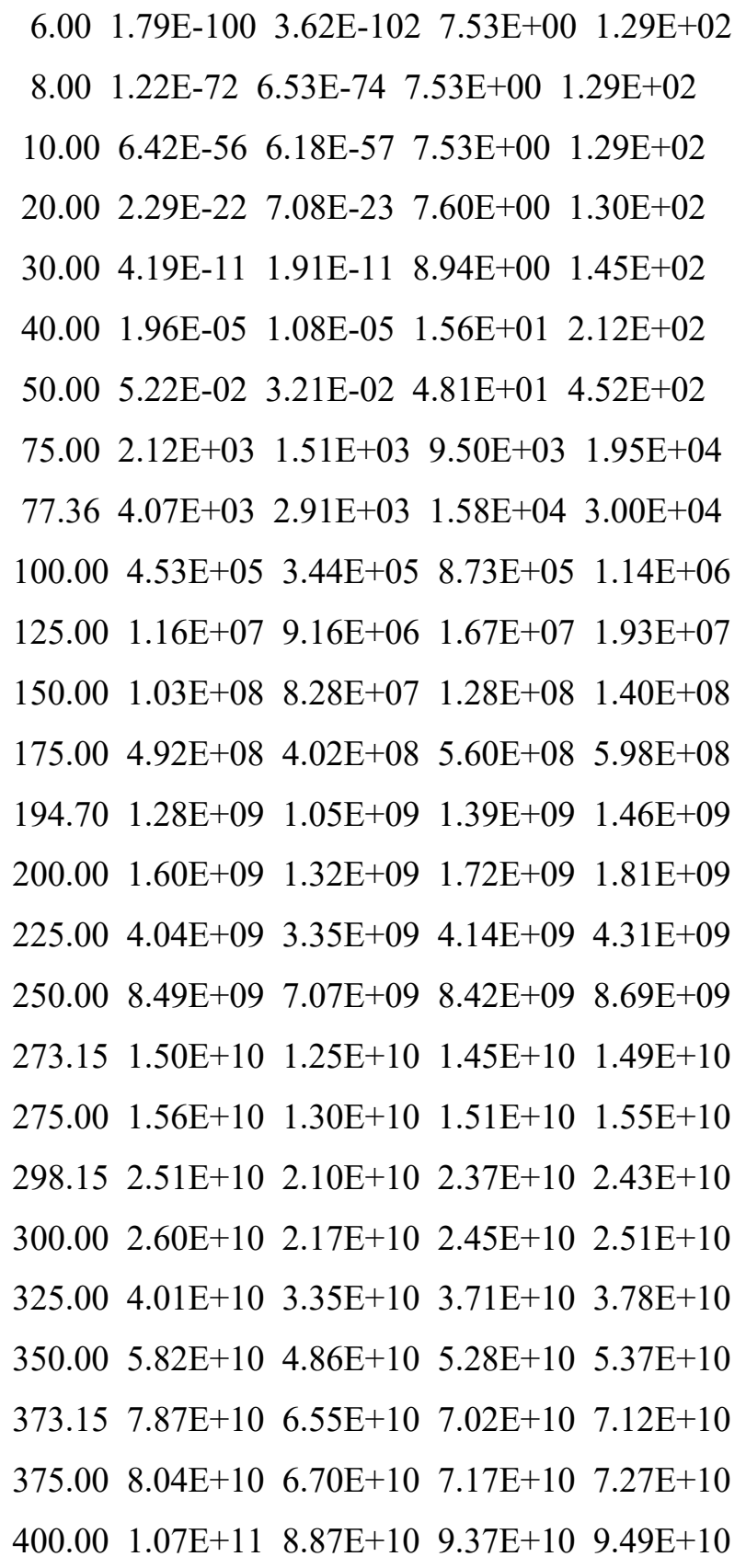

The following are VTST-ISPE calculation results.

Summary of forward rate constants $\left(\mathrm{s}^{-1}\right)$ :

$\mathrm{T}(\mathrm{K}) \quad \mathrm{TST} \quad \mathrm{CVT} \quad \mathrm{CVT} / \mathrm{ZCT} \quad \mathrm{CVT} / \mathrm{SCT}$

6.00 3.35E-124 9.90E-126 1.37E-01 5.21E+00

8.00 1.95E-90 1.39E-91 1.37E-01 5.21E+00

$10.00 \quad 3.73 \mathrm{E}-70 \quad 4.50 \mathrm{E}-71 \quad 1.37 \mathrm{E}-01 \quad 5.21 \mathrm{E}+00$ 
20.00 1.74E-29 6.04E-30 1.38E-01 5.24E+00

30.00 7.52E-16 3.70E-16 1.64E-01 5.73E+00

40.00 5.41E-09 3.16E-09 2.94E-01 7.84E+00

$50.00 \quad 7.42 \mathrm{E}-05 \quad 4.78 \mathrm{E}-05 \quad 9.24 \mathrm{E}-01 \quad 1.51 \mathrm{E}+01$

$\begin{array}{llll}75.00 & 2.68 \mathrm{E}+01 \quad 1.96 \mathrm{E}+01 \quad 2.13 \mathrm{E}+02 \quad 6.00 \mathrm{E}+02\end{array}$

$\begin{array}{lllll}77.36 & 5.86 \mathrm{E}+01 \quad 4.33 \mathrm{E}+01 \quad 3.79 \mathrm{E}+02 \quad 9.49 \mathrm{E}+02\end{array}$

$100.00 \quad 1.71 \mathrm{E}+04 \quad 1.33 \mathrm{E}+04 \quad 4.11 \mathrm{E}+04 \quad 5.88 \mathrm{E}+04$

$125.00 \quad 8.43 \mathrm{E}+05 \quad 6.78 \mathrm{E}+05 \quad 1.39 \mathrm{E}+06 \quad 1.68 \mathrm{E}+06$

$\begin{array}{llll}150.00 & 1.15 \mathrm{E}+07 \quad 9.45 \mathrm{E}+06 & 1.59 \mathrm{E}+07 \quad 1.78 \mathrm{E}+07\end{array}$

$\begin{array}{lll}175.00 & 7.54 \mathrm{E}+07 \quad 6.26 \mathrm{E}+07 \quad 9.30 \mathrm{E}+07 \quad 1.01 \mathrm{E}+08\end{array}$

$194.70 \quad 2.37 \mathrm{E}+08 \quad 1.98 \mathrm{E}+08 \quad 2.75 \mathrm{E}+08 \quad 2.94 \mathrm{E}+08$

$\begin{array}{llll}200.00 & 3.11 \mathrm{E}+08 \quad 2.60 \mathrm{E}+08 & 3.56 \mathrm{E}+08 & 3.78 \mathrm{E}+08\end{array}$

$\begin{array}{lllll}225.00 & 9.38 \mathrm{E}+08 & 7.89 \mathrm{E}+08 & 1.02 \mathrm{E}+09 & 1.07 \mathrm{E}+09\end{array}$

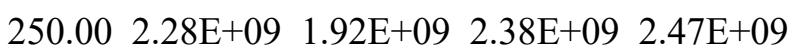

$273.15 \quad 4.50 \mathrm{E}+09 \quad 3.80 \mathrm{E}+09 \quad 4.56 \mathrm{E}+09 \quad 4.71 \mathrm{E}+09$

$275.00 \quad 4.73 \mathrm{E}+09 \quad 3.99 \mathrm{E}+09 \quad 4.78 \mathrm{E}+09 \quad 4.93 \mathrm{E}+09$

$298.15 \quad 8.35 \mathrm{E}+09 \quad 7.05 \mathrm{E}+09 \quad 8.22 \mathrm{E}+09 \quad 8.44 \mathrm{E}+09$

$300.00 \quad 8.70 \mathrm{E}+09 \quad 7.35 \mathrm{E}+09 \quad 8.56 \mathrm{E}+09 \quad 8.78 \mathrm{E}+09$

$\begin{array}{lllll}325.00 & 1.46 \mathrm{E}+10 & 1.23 \mathrm{E}+10 & 1.40 \mathrm{E}+10 & 1.43 \mathrm{E}+10\end{array}$

$\begin{array}{lllll}350.00 & 2.27 \mathrm{E}+10 & 1.92 \mathrm{E}+10 & 2.14 \mathrm{E}+10 & 2.18 \mathrm{E}+10\end{array}$

$\begin{array}{llll}373.15 & 3.26 \mathrm{E}+10 \quad 2.74 \mathrm{E}+10 \quad 3.01 \mathrm{E}+10 \quad 3.06 \mathrm{E}+10\end{array}$

$\begin{array}{llll}375.00 & 3.34 \mathrm{E}+10 \quad 2.81 \mathrm{E}+10 \quad 3.08 \mathrm{E}+10 \quad 3.14 \mathrm{E}+10\end{array}$

$400.00 \quad 4.69 \mathrm{E}+10 \quad 3.94 \mathrm{E}+10 \quad 4.25 \mathrm{E}+10 \quad 4.31 \mathrm{E}+10$

S-DR-d $\mathrm{d}_{6}$ to ret-CP- $\mathrm{d}_{6}$ :

Summary of forward rate constants $\left(\mathrm{s}^{-1}\right)$ :

T(K) TST CVT $\quad$ CVT/ZCT $\quad$ CVT/SCT

$6.00 \quad 1.10 \mathrm{E}-96 \quad 2.43 \mathrm{E}-97 \quad 6.43 \mathrm{E}-02 \quad 1.99 \mathrm{E}+00$

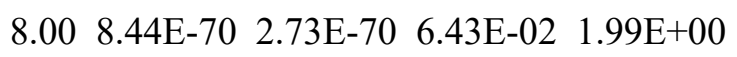

$10.00 \quad 1.20 \mathrm{E}-53 \quad 4.87 \mathrm{E}-54 \quad 6.43 \mathrm{E}-02 \quad 1.99 \mathrm{E}+00$

$20.00 \quad 3.14 \mathrm{E}-21 \quad 1.99 \mathrm{E}-21 \quad 6.69 \mathrm{E}-02 \quad 2.05 \mathrm{E}+00$

$30.00 \quad 2.41 \mathrm{E}-10 \quad 1.77 \mathrm{E}-10 \quad 1.02 \mathrm{E}-01 \quad 2.74 \mathrm{E}+00$

$40.00 \quad 7.32 \mathrm{E}-05 \quad 5.76 \mathrm{E}-05 \quad 3.71 \mathrm{E}-01 \quad 6.23 \mathrm{E}+00$

$50.00 \quad 1.50 \mathrm{E}-01 \quad 1.23 \mathrm{E}-01 \quad 4.87 \mathrm{E}+00 \quad 3.06 \mathrm{E}+01$ 


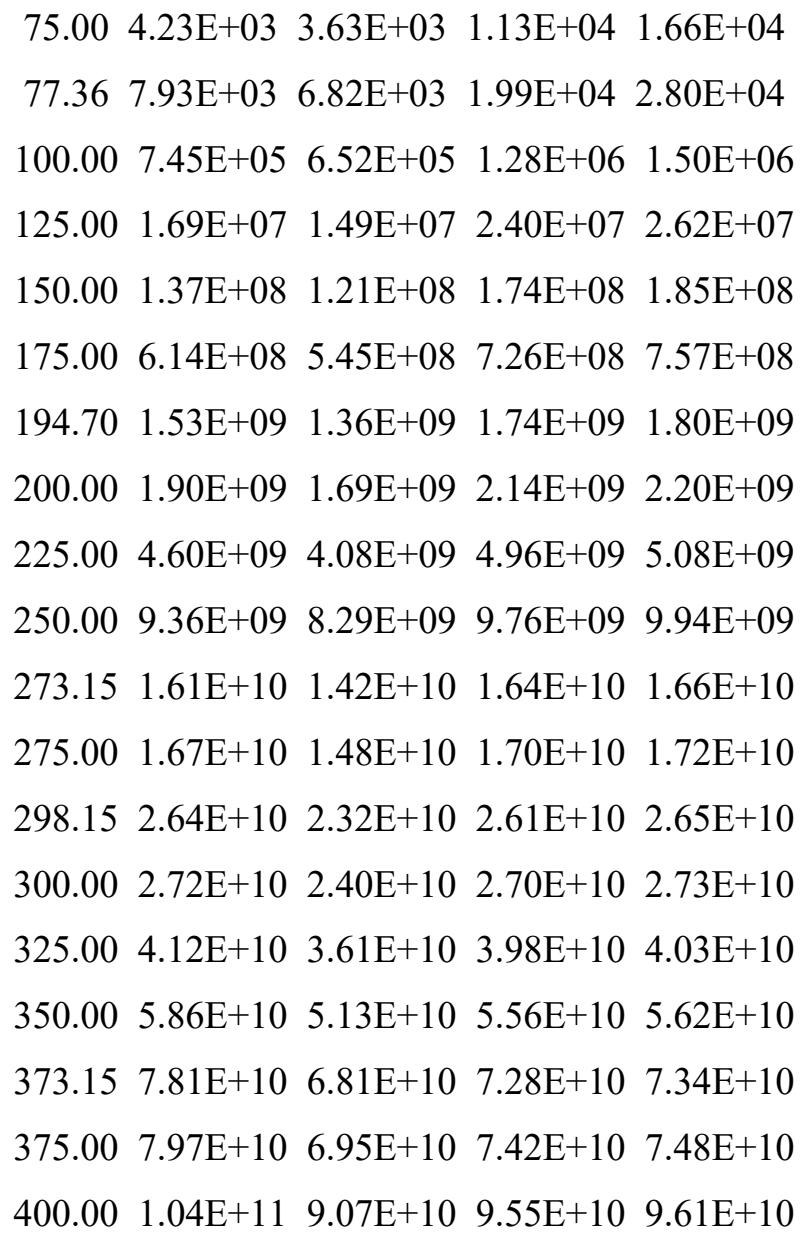

S-DR- $\mathrm{d}_{6}$ to inv-CP-d $\mathrm{d}_{6}$ :

Summary of forward rate constants $\left(\mathrm{s}^{-1}\right)$ :

$\mathrm{T}(\mathrm{K}) \quad \mathrm{TST} \quad \mathrm{CVT} \quad \mathrm{CVT} / \mathrm{ZCT} \quad \mathrm{CVT} / \mathrm{SCT}$

6.00 3.54E-100 5.93E-101 2.31E-02 7.55E-01

$8.00 \quad 2.03 \mathrm{E}-72 \quad 5.32 \mathrm{E}-73 \quad 2.31 \mathrm{E}-02 \quad 7.55 \mathrm{E}-01$

$10.00 \quad 9.68 \mathrm{E}-56 \quad 3.31 \mathrm{E}-56 \quad 2.31 \mathrm{E}-02 \quad 7.55 \mathrm{E}-01$

$20.00 \quad 2.81 \mathrm{E}-22 \quad 1.64 \mathrm{E}-22 \quad 2.41 \mathrm{E}-02 \quad 7.74 \mathrm{E}-01$

$30.00 \quad 4.82 \mathrm{E}-11 \quad 3.35 \mathrm{E}-11 \quad 3.75 \mathrm{E}-02 \quad 1.02 \mathrm{E}+00$

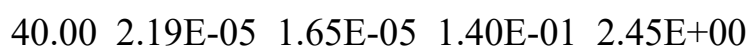

$50.00 \quad 5.71 \mathrm{E}-02 \quad 4.51 \mathrm{E}-02 \quad 1.93 \mathrm{E}+00 \quad 1.38 \mathrm{E}+01$

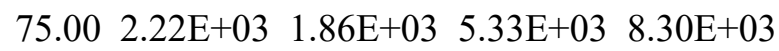

$\begin{array}{llll}77.36 & 4.25 \mathrm{E}+03 \quad 3.57 \mathrm{E}+03 \quad 9.46 \mathrm{E}+03 \quad 1.41 \mathrm{E}+04\end{array}$

$100.00 \quad 4.60 \mathrm{E}+05 \quad 3.95 \mathrm{E}+05 \quad 6.78 \mathrm{E}+05 \quad 8.28 \mathrm{E}+05$

$\begin{array}{llll}125.00 & 1.15 \mathrm{E}+07 \quad 1.00 \mathrm{E}+07 \quad 1.39 \mathrm{E}+07 \quad 1.57 \mathrm{E}+07\end{array}$

$\begin{array}{llll}150.00 & 9.95 \mathrm{E}+07 & 8.70 \mathrm{E}+07 & 1.09 \mathrm{E}+08 \quad 1.18 \mathrm{E}+08\end{array}$ 
$\begin{array}{lllll}175.00 & 4.68 \mathrm{E}+08 & 4.10 \mathrm{E}+08 & 4.79 \mathrm{E}+08 & 5.09 \mathrm{E}+08 \\ 194.70 & 1.20 \mathrm{E}+09 & 1.06 \mathrm{E}+09 & 1.19 \mathrm{E}+09 & 1.25 \mathrm{E}+09 \\ 200.00 & 1.50 \mathrm{E}+09 & 1.32 \mathrm{E}+09 & 1.47 \mathrm{E}+09 & 1.54 \mathrm{E}+09 \\ 225.00 & 3.74 \mathrm{E}+09 & 3.28 \mathrm{E}+09 & 3.55 \mathrm{E}+09 & 3.69 \mathrm{E}+09 \\ 250.00 & 7.76 \mathrm{E}+09 & 6.81 \mathrm{E}+09 & 7.21 \mathrm{E}+09 & 7.43 \mathrm{E}+09 \\ 273.15 & 1.36 \mathrm{E}+10 & 1.19 \mathrm{E}+10 & 1.24 \mathrm{E}+10 & 1.27 \mathrm{E}+10 \\ 275.00 & 1.41 \mathrm{E}+10 & 1.24 \mathrm{E}+10 & 1.29 \mathrm{E}+10 & 1.32 \mathrm{E}+10 \\ 298.15 & 2.26 \mathrm{E}+10 & 1.97 \mathrm{E}+10 & 2.02 \mathrm{E}+10 & 2.07 \mathrm{E}+10 \\ 300.00 & 2.34 \mathrm{E}+10 & 2.04 \mathrm{E}+10 & 2.09 \mathrm{E}+10 & 2.14 \mathrm{E}+10 \\ 325.00 & 3.57 \mathrm{E}+10 & 3.12 \mathrm{E}+10 & 3.15 \mathrm{E}+10 & 3.21 \mathrm{E}+10 \\ 350.00 & 5.15 \mathrm{E}+10 & 4.48 \mathrm{E}+10 & 4.47 \mathrm{E}+10 & 4.55 \mathrm{E}+10 \\ 373.15 & 6.91 \mathrm{E}+10 & 6.00 \mathrm{E}+10 & 5.93 \mathrm{E}+10 & 6.02 \mathrm{E}+10 \\ 375.00 & 7.07 \mathrm{E}+10 & 6.13 \mathrm{E}+10 & 6.06 \mathrm{E}+10 & 6.14 \mathrm{E}+10 \\ 400.00 & 9.32 \mathrm{E}+10 & 8.06 \mathrm{E}+10 & 7.89 \mathrm{E}+10 & 7.99 \mathrm{E}+10\end{array}$

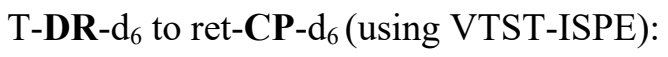

The following are VTST-ISPE calculation results.

Summary of forward rate constants $\left(\mathrm{s}^{-1}\right)$ :

T(K) TST CVT CVT/ZCT CVT/SCT

$6.00 \quad$ 2.37E-120 6.52E-121 6.04E-04 2.57E-02

$8.00 \quad 1.51 \mathrm{E}-87 \quad 5.71 \mathrm{E}-88 \quad 6.04 \mathrm{E}-04 \quad 2.57 \mathrm{E}-02$

$10.00 \quad 7.61 \mathrm{E}-68 \quad 3.50 \mathrm{E}-68 \quad 6.04 \mathrm{E}-04 \quad 2.57 \mathrm{E}-02$

$20.00 \quad 2.49 \mathrm{E}-28 \quad 1.69 \mathrm{E}-28 \quad 6.31 \mathrm{E}-04 \quad 2.66 \mathrm{E}-02$

$30.00 \quad 4.45 \mathrm{E}-15 \quad 3.42 \mathrm{E}-15 \quad 1.01 \mathrm{E}-03 \quad 3.77 \mathrm{E}-02$

$40.00 \quad 2.06 \mathrm{E}-08 \quad 1.68 \mathrm{E}-08 \quad 3.78 \mathrm{E}-03 \quad 9.57 \mathrm{E}-02$

$50.00 \quad 2.16 \mathrm{E}-04$ 1.82E-04 4.33E-02 4.97E-01

$\begin{array}{llll}75.00 & 5.40 \mathrm{E}+01 \quad 4.73 \mathrm{E}+01 \quad 1.96 \mathrm{E}+02 \quad 3.60 \mathrm{E}+02\end{array}$

$\begin{array}{llll}77.36 & 1.16 \mathrm{E}+02 \quad 1.01 \mathrm{E}+02 \quad 3.78 \mathrm{E}+02 \quad 6.53 \mathrm{E}+02\end{array}$

$100.00 \quad 2.83 \mathrm{E}+04 \quad 2.51 \mathrm{E}+04 \quad 5.28 \mathrm{E}+04 \quad 6.82 \mathrm{E}+04$

$\begin{array}{lll}125.00 & 1.23 \mathrm{E}+06 \quad 1.10 \mathrm{E}+06 \quad 1.77 \mathrm{E}+06 \quad 2.06 \mathrm{E}+06\end{array}$

$\begin{array}{llllll}150.00 & 1.54 \mathrm{E}+07 & 1.38 \mathrm{E}+07 & 1.93 \mathrm{E}+07 & 2.13 \mathrm{E}+07\end{array}$

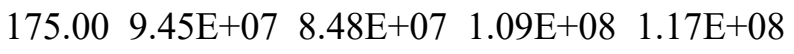

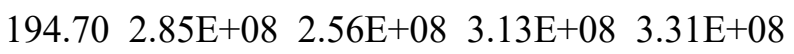

$200.00 \quad 3.70 \mathrm{E}+08 \quad 3.32 \mathrm{E}+08 \quad 4.01 \mathrm{E}+08 \quad 4.23 \mathrm{E}+08$ 


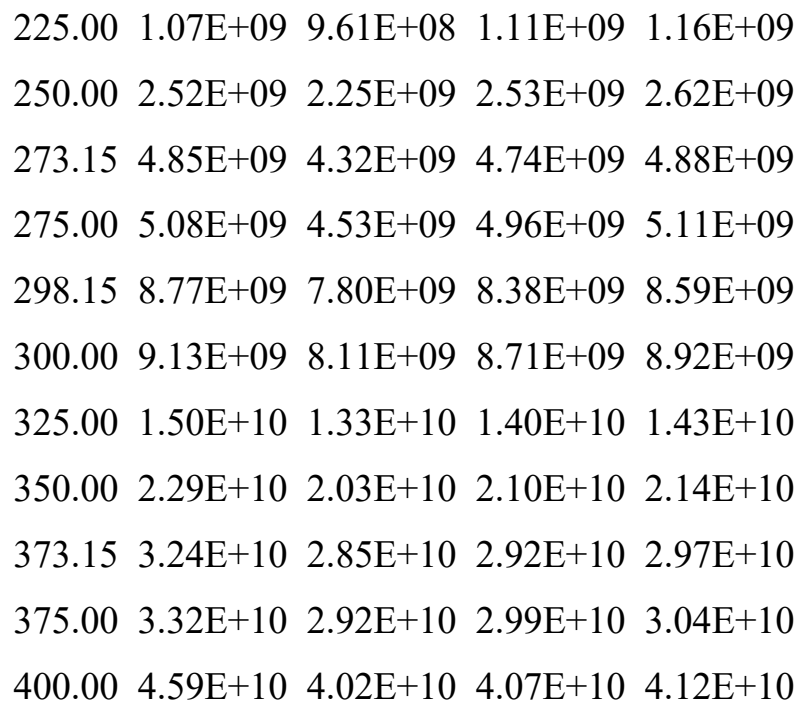

T-DR-d ${ }_{6}$ to inv-CP-d d $_{6}$ (using VTST-ISPE):

The following are VTST-ISPE calculation results.

Summary of forward rate constants $\left(\mathrm{s}^{-1}\right)$ :

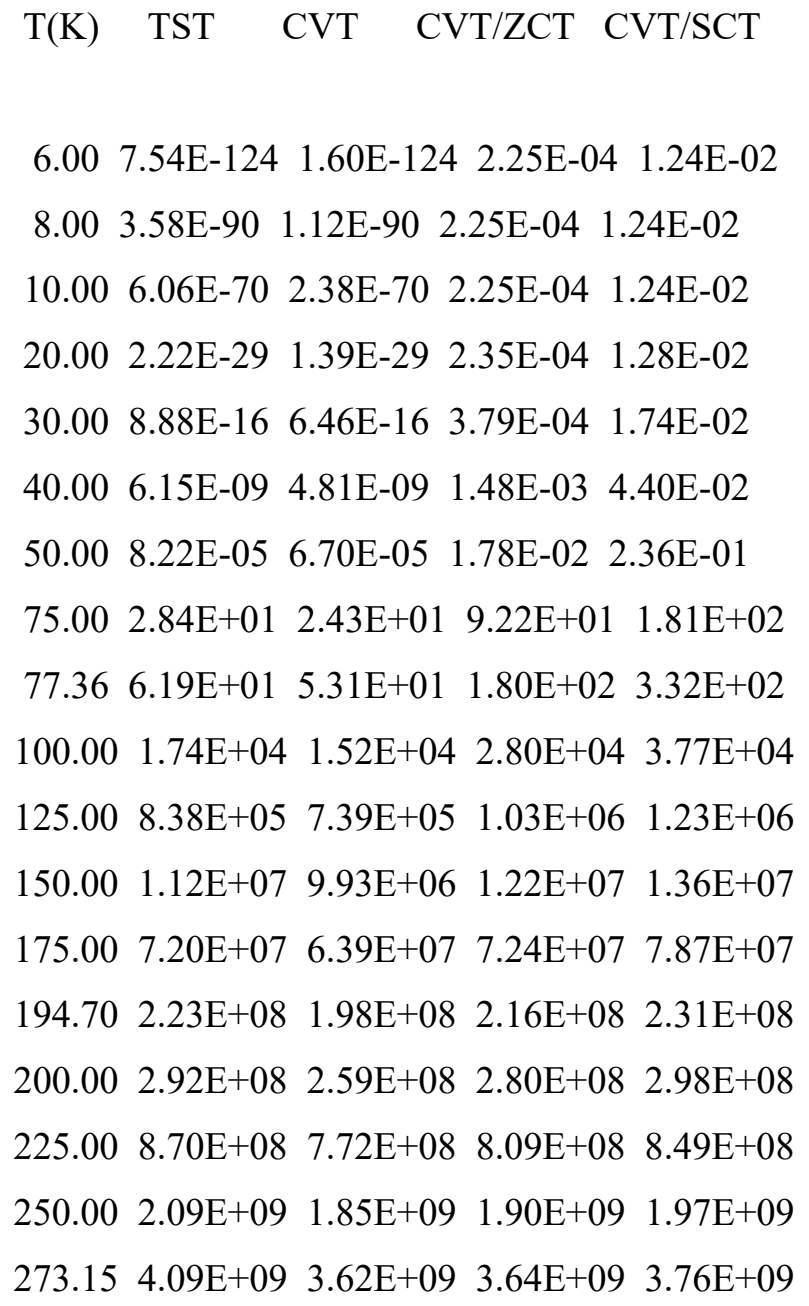




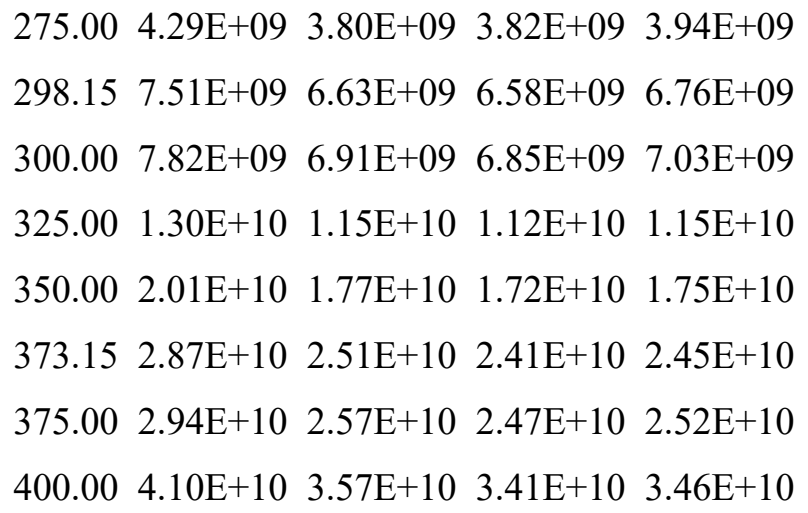

$\mathrm{S}_{\mathrm{H}} 2$ reaction from $\mathrm{S}-\mathbf{1}$ to $\mathbf{C P}$ :

Summary of forward rate constants $\left(\mathrm{s}^{-1}\right)$ :

\section{T(K) TST CVT $\quad$ CVT/ZCT $\quad$ CVT/SCT}

6.00 4.99E-61 4.82E-61 8.77E-12 7.44E-11

$8.00 \quad 4.76 \mathrm{E}-43$ 4.64E-43 8.77E-12 7.44E-11

$10.00 \quad 3.07 \mathrm{E}-32 \quad 3.01 \mathrm{E}-32 \quad 8.88 \mathrm{E}-12 \quad 7.50 \mathrm{E}-11$

20.00 1.64E-10 1.62E-10 4.82E-09 1.17E-08

$30.00 \quad 3.39 \mathrm{E}-03 \quad 3.37 \mathrm{E}-03 \quad 1.13 \mathrm{E}-02 \quad 1.34 \mathrm{E}-02$

$40.00 \quad 1.67 \mathrm{E}+01 \quad 1.66 \mathrm{E}+01 \quad 3.75 \mathrm{E}+01 \quad 4.12 \mathrm{E}+01$

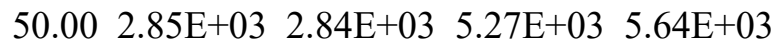

$75.00 \quad 2.90 \mathrm{E}+06 \quad 2.88 \mathrm{E}+06 \quad 4.21 \mathrm{E}+06 \quad 4.40 \mathrm{E}+06$

$\begin{array}{lll}77.36 & 4.43 \mathrm{E}+06 \quad 4.41 \mathrm{E}+06 \quad 6.35 \mathrm{E}+06 \quad 6.62 \mathrm{E}+06\end{array}$ $\begin{array}{lll}100.00 & 9.60 \mathrm{E}+07 \quad 9.53 \mathrm{E}+07 \quad 1.25 \mathrm{E}+08 \quad 1.29 \mathrm{E}+08\end{array}$ $\begin{array}{lll}125.00 & 7.94 \mathrm{E}+08 \quad 7.88 \mathrm{E}+08 \quad 9.68 \mathrm{E}+08 \quad 9.92 \mathrm{E}+08\end{array}$

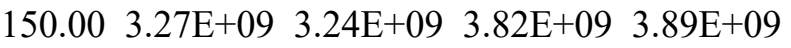

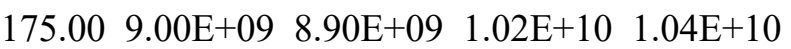
$194.70 \quad 1.67 \mathrm{E}+10 \quad 1.65 \mathrm{E}+10 \quad 1.85 \mathrm{E}+10 \quad 1.88 \mathrm{E}+10$ $200.00 \quad 1.93 \mathrm{E}+10 \quad 1.90 \mathrm{E}+10 \quad 2.14 \mathrm{E}+10 \quad 2.17 \mathrm{E}+10$ $\begin{array}{lllll}225.00 & 3.48 \mathrm{E}+10 & 3.43 \mathrm{E}+10 & 3.79 \mathrm{E}+10 & 3.84 \mathrm{E}+10\end{array}$ $250.00 \quad 5.60 \mathrm{E}+10 \quad 5.51 \mathrm{E}+10 \quad 6.01 \mathrm{E}+10 \quad 6.07 \mathrm{E}+10$ $273.15 \quad 8.04 \mathrm{E}+10 \quad 7.90 \mathrm{E}+10 \quad 8.53 \mathrm{E}+10 \quad 8.61 \mathrm{E}+10$ $275.00 \quad 8.26 \mathrm{E}+10 \quad 8.11 \mathrm{E}+10 \quad 8.75 \mathrm{E}+10 \quad 8.83 \mathrm{E}+10$ $298.15 \quad 1.12 \mathrm{E}+11 \quad 1.09 \mathrm{E}+11 \quad 1.17 \mathrm{E}+11 \quad 1.18 \mathrm{E}+11$ $300.00 \quad 1.14 \mathrm{E}+11 \quad 1.12 \mathrm{E}+11 \quad 1.20 \mathrm{E}+11 \quad 1.21 \mathrm{E}+11$ $325.00 \quad 1.50 \mathrm{E}+11 \quad 1.47 \mathrm{E}+11 \quad 1.56 \mathrm{E}+11 \quad 1.57 \mathrm{E}+11$ $350.00 \quad 1.90 \mathrm{E}+11 \quad 1.86 \mathrm{E}+11 \quad 1.96 \mathrm{E}+11 \quad 1.97 \mathrm{E}+11$ 
$373.15 \quad 2.29 \mathrm{E}+11 \quad 2.24 \mathrm{E}+11 \quad 2.35 \mathrm{E}+11 \quad 2.36 \mathrm{E}+11$

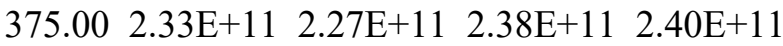

$\begin{array}{llll}400.00 & 2.78 \mathrm{E}+11 & 2.71 \mathrm{E}+11 \quad 2.82 \mathrm{E}+11 & 2.84 \mathrm{E}+11\end{array}$

\section{Example of Polyrate Input Files}

S-DR to CP (no ISPE):

.70 file:

*GRGENERAL

GRRESTART

*GRSTART

CHARGE 0

MULTIPLICITY 1

${ }^{*}$ GRCOMMON

GRENER

$\%$ mem $=100 \mathrm{gb}$

$\%$ nproc $=16$

\#n ub3lyp/6-31g(d) units(au) fchk nosymm int(ultrafine) guess=cards punch=mo

END

\section{GRFIRST}

$\%$ mem $=100 \mathrm{gb}$ 
$\%$ nproc $=16$

\#n ub3lyp/6-31g(d) units(au) fchk nosymm force int(ultrafine) guess=cards

END

GRSEC

$\%$ mem $=100 \mathrm{gb}$

$\%$ nproc $=16$

\#n ub3lyp/6-31g(d) units(au) fchk nosymm freq int(ultrafine) guess=cards

END

\section{GRBASIS}

@ $/$ local/gaus/kozuch/s-dr_to_cp/s-dr_to_cp_ts_vt_pun.pun

END

.71 file:

$\%$ mem $=100 \mathrm{gb}$

$\%$ nproc $=16$

\#n ub3lyp/6-31g(d) fchk nosymm int(ultrafine) guess=cards

01

$\begin{array}{llll}\text { C } & -0.000000 & 0.000000 & -1.343197\end{array}$

H $\quad 0.874762 \quad 0.004635 \quad-2.022868$

H $\quad-0.874762-0.004635 \quad-2.022868$

C $\quad-0.000000 \quad-1.177360 \quad-0.408442$

H $\quad 0.028843 \quad-2.209680 \quad-0.742361$

$\begin{array}{llll}\text { C } & -0.000000 & 1.177360 & -0.408442\end{array}$

H $\quad-0.028843 \quad 2.209680 \quad-0.742361$

$\begin{array}{llll}\text { C } & -0.142467 & 0.764219 & 1.024871\end{array}$

H $\quad-1.163337 \quad 0.962017 \quad 1.398781$

$\begin{array}{llll}\mathrm{H} & 0.528823 & 1.317017 & 1.697468\end{array}$

C $\quad 0.142467 \quad-0.764219 \quad 1.024871$

H $\quad \begin{array}{llll}1.163337 & -0.962017 & 1.398781\end{array}$

$\begin{array}{llll}\mathrm{H} & -0.528823 & -1.317017 & 1.697468\end{array}$

@ $/$ local/gaus/kozuch/s-dr_to_cp/s-dr_vt_pun.pun 
.73 file:

$\%$ mem $=100 \mathrm{gb}$

$\%$ nproc $=16$

\#n b3lyp/6-31g(d) fchk nosymm int(ultrafine)
01
C $\quad 1.323672 \quad-0.000000 \quad 0.491293$
H $\quad 1.152530 \quad-0.000001 \quad 1.568916$
H $\quad 2.374359-0.000000 \quad 0.210681$
C $\quad 0.410323 \quad-0.766206-0.425653$
H $\quad 0.798944 \quad-1.448952-1.176271$
$\begin{array}{llll}\text { C } & 0.410323 & 0.766207 & -0.425652\end{array}$
H $\quad 0.798944 \quad 1.448954 \quad-1.176269$
$\begin{array}{llll}\text { C } & -1.016770 & 0.783020 & 0.137030\end{array}$
$\mathrm{H} \quad-1.750701 \quad 1.193995 \quad-0.562465$

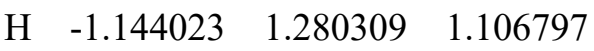
$\begin{array}{llll}\text { C } & -1.016770 & -0.783021 & 0.137030\end{array}$
H $\quad-1.144021 \quad-1.280310 \quad 1.106797$
H $\quad-1.750701 \quad-1.193996 \quad-0.562465$

.75 file:

$\%$ mem $=100 \mathrm{gb}$

$\%$ nproc $=16$

\#n ub3lyp/6-31g(d) guess=cards fchk nosymm int(ultrafine)
01
C $\quad-1.334298 \quad-0.000000 \quad 0.239031$
H $\quad-1.568306 \quad-0.000000 \quad 1.323589$
H $\quad-2.295056-0.000000 \quad-0.289913$
$\begin{array}{llll}\text { C } & -0.421503 & 1.116647 & -0.188740\end{array}$
$\mathrm{H} \quad-0.744553 \quad 1.965322 \quad-0.783016$
C $\quad-0.421503 \quad-1.116647 \quad-0.188740$ 

H $\quad-0.744553 \quad-1.965322 \quad-0.783016$
$\begin{array}{llll}\text { C } & 1.022259 & -0.778614 & 0.063978\end{array}$
H $\quad 1.680401-1.174812-0.718682$
H $\quad 1.394188-1.195315 \quad 1.016336$
C $\quad 1.022259 \quad 0.778614 \quad 0.063978$
H $\quad 1.394188 \quad 1.195315 \quad 1.016336$
H $\quad 1.680401 \quad 1.174812 \quad-0.718682$

@ $/$ local/gaus/kozuch/s-dr_to_cp/s-dr_to_cp_ts_vt_pun.pun

dat file:

*GENERAL

TITLE

s-dr_to_cp

END

\section{ATOMS}

$1 \mathrm{C}$

$2 \mathrm{H}$

$3 \mathrm{H}$

$4 \mathrm{C}$

$5 \mathrm{H}$

$6 \mathrm{C}$

$7 \mathrm{H}$

$8 \mathrm{C}$

$9 \mathrm{H}$

$10 \mathrm{H}$

$11 \mathrm{C}$

$12 \mathrm{H}$

$13 \mathrm{H}$

END

NOSUPERMOL 
*SECOND

HESSCAL hhook

FPRINT

*OPTIMIZATION

PRINT

OPTMIN ohook

OPTTS ohook

*REACT1

INITGEO hooks

GEOM

1

2

3

4

5

6

7

8

9

10

11

12

13

END

SPECIES nonlinrp

*PROD1

INITGEO hooks

GEOM

1

2 
8

9

10

11

12

13

END

SPECIES nonlinrp

*START

INITGEO hooks

GEOM

1

2

3

4

5

6

7

8

9

10

11

12

13

END

SPECIES nonlints

PROJECT

*PATH

\#SYMMETRY 
INTMU 3

SSTEP 0.0005

RPM pagem

SRANGE

SLP 3.

SLM -2.9

END

\#SPECSTOP

\# CURVE VMEP

\# PERCENTDOWN 99.8

\#END

PRPATH

coord 12

xmol

freq 33

END

*TUNNEL

ZCT

SCT

QRST

harmonic

mode 33

states all

END

*RATE

FORWARDK

SIGMAF 1

TST

CVT

PRDELG

PRPART rtp

TEMP

6

8 
10

20

30

40

50

75

77.355

100

125

150

175

194.7

200

225

250

273.15

275

298.15

300

325

350

373.15

375

400

END

\section{ANALYSIS}

6

8

10

20

30

40

50

75

77.355

100 
125

150

175

194.7

200

225

250

273.15

275

298.15

300

325

350

373.15

375

400

END

EACT

6. 10 .

10. 20.

20. 50 .

50. 100 .

200. 225 .

300. 325 .

END

GTLOG

S-DR to CP (with VTST-ISPE):

.70 file:

*GRGENERAL

GRRESTART

*GRSTART 
CHARGE 0

MULTIPLICITY 1

*GRCOMMON

GRENER

$\%$ mem $=100 \mathrm{gb}$

$\%$ nproc $=16$

\#n ub3lyp/6-31g(d) units(au) fchk nosymm int(ultrafine) guess $=$ cards punch=mo

END

\section{GRFIRST}

$\%$ mem $=100 \mathrm{gb}$

$\%$ nproc $=16$

\#n ub3lyp/6-31g(d) units(au) fchk nosymm force int(ultrafine) guess=cards

END

\section{GRSEC}

$\%$ mem $=100 \mathrm{gb}$

$\%$ nproc $=16$

\#n ub3lyp/6-31g(d) units(au) fchk nosymm freq int(ultrafine) guess=cards

END

\section{GRBASIS}

@ $/$ local/gaus/kozuch/s-dr_to_cp_ISPE/s-dr_to_cp_ts_vt_pun.pun

END

.71 file:

$\%$ mem $=100 \mathrm{gb}$

$\%$ nproc $=16$

\#n ub3lyp/6-31g(d) fchk nosymm int(ultrafine) guess=cards

$\begin{array}{llll}0 & 1 \\ \text { C } & -0.000000 & 0.000000 & -1.343197\end{array}$ 

$\mathrm{H} \quad 0.874762 \quad 0.004635 \quad-2.022868$
H $\quad-0.874762-0.004635 \quad-2.022868$
$\begin{array}{llll}\text { C } & -0.000000 & -1.177360 & -0.408442\end{array}$
H $\quad 0.028843 \quad-2.209680 \quad-0.742361$
$\begin{array}{llll}\text { C } & -0.000000 & 1.177360 & -0.408442\end{array}$
$\mathrm{H} \quad-0.028843 \quad 2.209680-0.742361$
$\begin{array}{llll}\text { C } & -0.142467 & 0.764219 & 1.024871\end{array}$
$\mathrm{H} \quad-1.163337 \quad 0.962017 \quad 1.398781$
$\mathrm{H} \quad 0.528823 \quad 1.317017 \quad 1.697468$
C $\quad 0.142467 \quad-0.764219 \quad 1.024871$
H $\quad 1.163337 \quad-0.962017 \quad 1.398781$
$\begin{array}{llll}\mathrm{H} & -0.528823 & -1.317017 & 1.697468\end{array}$

@/local/gaus/kozuch/s-dr_to_cp_ISPE/s-dr_vt_pun.pun

.73 file:

$\%$ mem $=100 \mathrm{gb}$

$\%$ nproc $=16$

\#n b31yp/6-31g(d) fchk nosymm int(ultrafine)
01
C $\quad 1.323672 \quad-0.000000 \quad 0.491293$
$\mathrm{H} \quad 1.152530 \quad-0.000001 \quad 1.568916$
H $\quad 2.374359 \quad-0.000000 \quad 0.210681$
C $\quad 0.410323 \quad-0.766206-0.425653$
H $\quad 0.798944 \quad-1.448952-1.176271$
$\begin{array}{llll}\text { C } & 0.410323 & 0.766207 & -0.425652\end{array}$
H $\quad 0.798944 \quad 1.448954 \quad-1.176269$
$\begin{array}{llll}\text { C } & -1.016770 & 0.783020 & 0.137030\end{array}$
$\begin{array}{llll}\mathrm{H} & -1.750701 & 1.193995 & -0.562465\end{array}$

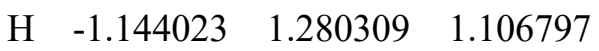
$\begin{array}{llll}\text { C } & -1.016770 & -0.783021 & 0.137030\end{array}$
Н $\quad-1.144021 \quad-1.280310 \quad 1.106797$
H $\quad-1.750701-1.193996-0.562465$ 
.75 file:

$\%$ mem $=100 \mathrm{gb}$

$\%$ nproc $=16$

\#n ub3lyp/6-31g(d) guess=cards fchk nosymm int(ultrafine)
01
C $\quad-1.334298 \quad-0.000000 \quad 0.239031$
$\mathrm{H} \quad-1.568306-0.000000 \quad 1.323589$
H $\quad-2.295056-0.000000 \quad-0.289913$
C $\quad-0.421503 \quad 1.116647 \quad-0.188740$
$\mathrm{H} \quad-0.744553 \quad 1.965322-0.783016$
C $\quad-0.421503 \quad-1.116647-0.188740$
H $\quad-0.744553 \quad-1.965322 \quad-0.783016$
C $\quad \begin{array}{lll}1.022259 & -0.778614 & 0.063978\end{array}$
H $\quad 1.680401-1.174812-0.718682$
H $\quad 1.394188 \quad-1.195315 \quad 1.016336$
$\begin{array}{llll}\text { C } & 1.022259 & 0.778614 & 0.063978\end{array}$
H $\quad 1.394188 \quad 1.195315 \quad 1.016336$
H $\quad 1.680401 \quad 1.174812-0.718682$

@ $/$ local/gaus/kozuch/s-dr_to_cp_ISPE/s-dr_to_cp_ts_vt_pun.pun

.51 file:

*ISPEGEN

ENERXN -31.58 \# Corrected Exergonicity

ENESAD 3.67 \# Corrected Activation energy

MEPTYPER one

MEPTYPEP one

RCINFO

SRC -5.4764 \# Position of the reactant in Bohr (s from fu28)

END 
PCINFO

SPC 5.6635 \# Position of the product in Bohr

END

.dat file:

*GENERAL

TITLE

s-dr_to_cp

END

DL ISPE

ATOMS

$1 \mathrm{C}$

$2 \mathrm{H}$

$3 \mathrm{H}$

$4 \mathrm{C}$

$5 \mathrm{H}$

$6 \mathrm{C}$

$7 \mathrm{H}$

$8 \mathrm{C}$

$9 \mathrm{H}$

$10 \mathrm{H}$

$11 \mathrm{C}$

$12 \mathrm{H}$

$13 \mathrm{H}$

END

NOSUPERMOL

*SECOND

HESSCAL hhook

FPRINT 
*OPTIMIZATION

PRINT

OPTMIN ohook

OPTTS ohook

*REACT1

INITGEO hooks

GEOM

1

2

3

4

5

6

7

8

9

10

11

12

13

END

SPECIES nonlinrp

*PROD1

INITGEO hooks

\section{GEOM}

1

2

3

4

5

6

7 
12

13

END

SPECIES nonlinrp

*START

INITGEO hooks

GEOM

1

2

3

4

5

6

7

8

9

10

11

12

13

END

SPECIES nonlints

PROJECT

*PATH

\#SYMMETRY

INTMU 3

SSTEP 0.0005

RPM pagem

SRANGE

SLP 3.

SLM -2.9 
END

\#SPECSTOP

\# CURVE VMEP

\# PERCENTDOWN 99.8

\#END

PRPATH

coord 12

xmol

freq 33

END

*TUNNEL

ZCT

SCT

QRST

harmonic

mode 33

states all

END

*RATE

FORWARDK

SIGMAF 1

TST

CVT

PRDELG

PRPART rtp

\section{TEMP}

6

8

10

20

30

40

50

75 
77.355

100

125

150

175

194.7

200

225

250

273.15

275

298.15

300

325

350

373.15

375

400

END

\section{ANALYSIS}

6

8

10

20

30

40

50

75

77.355

100

125

150

175

194.7

200

225 
END

\section{EACT}

6. 10 .

10. 20.

20. 50 .

50. 100 .

200. 225.

300. 325.

END

\section{GTLOG}

\section{References}

1. Bauer, S. H. Operational Criteria for Concerted Bond Breaking in Gas-phase Molecular Elimination Reactions. J. Am. Chem. Soc. 1969, 91, 3688-3689.

2. Adams, J. S.; Weisman, R. B.; Engel, P. S. Photodissociation of a Bicyclic azoalkane: Timeresolved Coherent Anti-Stokes Raman Spectroscopy Studies of Vapor-phase 2,3Diazabicyclo[2.2.1] hept-2-ene. J. Am. Chem. Soc. 1990, 112, 9115-9121.

3. Simpson, C. J. S. M.; Wilson, G. J.; Adam, W. Dynamics of the Thermal Decomposition of 2,3-Diazabicyclo[2.2.1]hept-2-ene. J. Am. Chem. Soc. 1991, 113, 4728-4732.

4. Yamamoto, N.; Olivucci, M.; Celani, P.; Bernardi, F.; Robb, M. A. An MC-SCF/MP2 Study of the Photochemistry of 2,3-Diazabicyclo[2.2.1]hept-2-ene: Production and Fate of Diazenyl and Hydrazonyl Biradicals. J. Am. Chem. Soc. 1998, 120, 2391-2407. 
5. Sinicropi, A.; Page, C. S.; Adam, W.; Olivucci, M. Computational Study on the Origin of the Stereoselectivity for the Photochemical Denitrogenation of Diazabicycloheptene. J. Am. Chem. Soc. 2003, 125, 10947-10959.

6. Abe, M. Diradicals. Chem. Rev. 2013, 113, 7011-7088.

7. Schreiner, P. R.; Wagner, J. P.; Reisenauer, H. P.; Gerbig, D.; Ley, D.; Sarka, J.; Császár, A. G.; Vaughn, A.; Allen, W. D. Domino Tunneling. J. Am. Chem. Soc. 2015, 137, 7828-7834.

8. Siebrand, W.; Wildman, T. A. Dispersive Kinetics: a Structural Approach to Nonexponential Processes in Disordered Media. Acc. Chem. Res. 1986, 19, 238-243.

9. Adam, W.; Mazenod, F. Facile Liquid-phase Denitrogenation of "Reluctant" Azoalkanes on Photolysis with 185-nm Radiation. J. Am. Chem. Soc. 1980, 102, 7131-7132.

10. Adam, W.; Maas, W.; Nau, W. M. Wavelength-Selective Photodenitrogenation of Azoalkanes to High-Spin Polyradicals with Cyclopentane-1,3-diyl Spin-Carrying Units and Their Photobleaching: EPR/UV Spectroscopy and Product Studies of the Matrix-Isolated Species. The Journal of Organic Chemistry 2000, 65, 8790-8796.

11. Solomon, B. S.; Thomas, T. F.; Steel, C. Primary Processes in the Photochemistry of Diazabicyclic Compounds. J. Am. Chem. Soc. 1968, 90, 2249-2258.

12. Adam, W.; Fragale, G.; Klapstein, D.; Nau, W. M.; Wirz, J. Phosphorescence and Transient Absorption of Azoalkane Triplet States. J. Am. Chem. Soc. 1995, 117, 12578-12592. 